\title{
NBSIR 81-2280
}

\section{Technical Activities of the Office of Environmental Measurements--1980}

William H. Kirchhoff, Chief

Office of Environmental Measurements

National Measurement Laboratory

U.S. Department of Commerce

National Bureau of Standards

Washington, DC 20234

May 1981

Prepared for:

National Bureau of Standards QC epartment of Commerce 

William H. Kirchhoff, Chief

Office of Enviornmental Measurements

National Measurement Laboratory

U.S. Department of Commerce

National Bureau of Standards

Washington, DC 20234

May 1981

Prepared for:

National Bureau of Standards

Department of Commerce

Washington, DC 20234

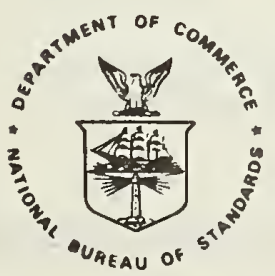

U.S. DEPARTMENT OF COMMERCE, Malcolm Baldrige, Secretary NATIONAL BUREAU OF STANDARDS, Ernest Ambler, Director 



\section{TECHNICAL ACTIVITIES}

Table of Contents

Disclaimer................................................ i

Abstract.......................................................... ii

Introduction.................................................i

WATER MEASUREMENTS - STANDARDS DEVELOPMENT-_-1.-1

Standard Reference Materials for Organics in Water: Project Description-------3

Selected Technical Accomplishments

Certification of Generator Columns for Polynuclear Aromatic

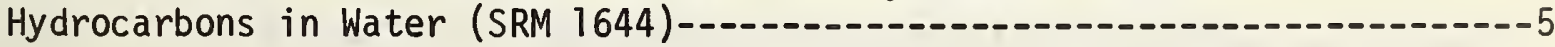

Determined Aqueous Solubilities and Chromatographic Characteristics for Organic Compounds on the Priority Pollutant List--.---

Developed and Evaluated a Quenchofluorometric Detection System for

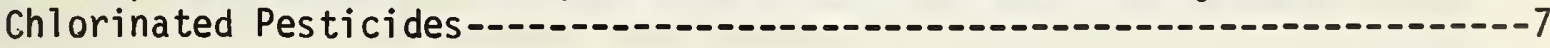

Liquid Chromatographic Retention Characteristics of Polycyclic Aromatic Hydrocarbons---_-_-_-

Stability Study of Halohydrocarbons---10-14

Developed Alternate Proposal for Quality Assurance of PAH Measurement in Water--1-.--1

Environmental Radioactivity Measurements: Project Description--_-_-_-1-18---18

Selected Technical Accomplishment

Provided Services to EPA/Las Vegas Under the Measurement Traceability

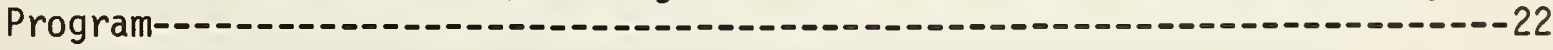

Evaluated Monitoring Methods for Radium-228 and Issued a Report---------23

Developed a Sampling Procedure for Calibration of ${ }^{133}$ Xe-Air Mixtures----23

Seven Natural Matrix Materials Have Been Dried, Ground, Homogenized,

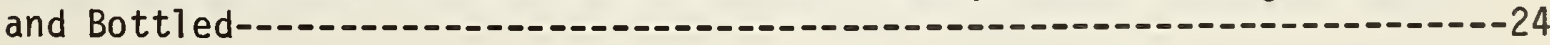

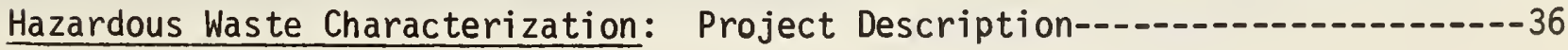

Selected Technical Accomplishment

Characterization of Potentially Hazardous Waste-Development of Rotary Extractor and Quality Assurance Standards 
Energy-Related Organic and Inorganic Water Standards Development:

Project Description

\section{Selected Technical Accomplishments}

First Natural Matrix Trace Organic SRM Issued for Shale 011 SRM 1580---42

GC/MS Analysis of Aqueous Effluents from Coal Conversion Processes-----45

Application of Multi-Dimensional Chromatography to Trace Organic

Compound Quantitation--

Combined LC/MS Technique for Direct Quantitative Analysis for

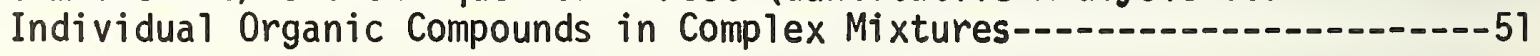

Work Plan and Evaluation/Feasibility Study of Drilling Fluid

Reference Standards for Chemical Analys is-_-_._-_...-. 52

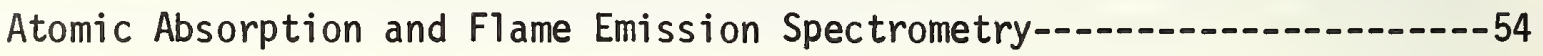

Metal Speciation Using High Performance Liquid Chromatography/Atomic

Absorption Spectrometry---

Interferences in the Inductively Coupled Plasma (ICP) Technique--------60

Radiological Pollutant Quality Assurance: Project Description--------------61

Accomplishments

${ }^{232}$ Th Solution--_-

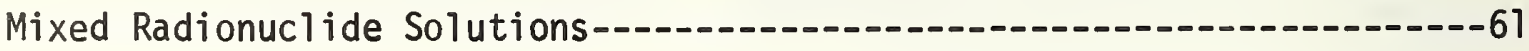


Methods Development for Organic Analysis: Project Description-------------72

Selected Techncial Accomplishments

Synthesis, Isotopic and Purity Analyses, and Preparation of Solutions of Stable Isotope Labeled Marker Compounds for

Priority Pollutant Analysis--_-

Fluoroimmunoassay Procedure Developed for Dinitrophenols in Water-----74

Correlation of Liquid Chromatographic Retention Data. with Molecular

Shapes for Polycyclic Aromatic Hydrocarbons--.--.--.--75

Liquid Chromatography with Electrochemical Detection (LC-EC)----------76

Development of a Fourier Transform Infrared Spectrometer-Gas

Chromatograph Interface and Its Application to Pollutant

Identification---

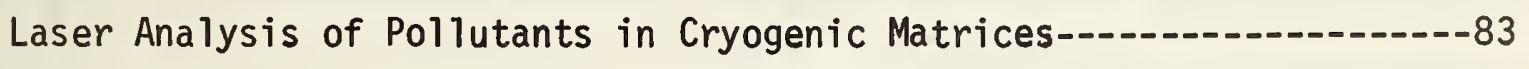

Methods Development for Inorganic Analysis; Project Description----_-------84

Selected Technical Accomplishments

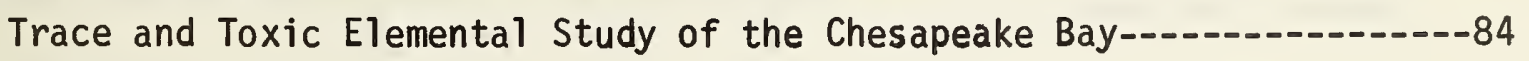

Sulfur Speciation in Coal-_-

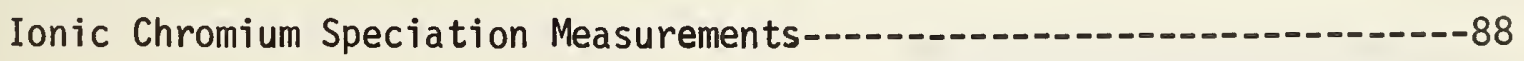

AIR MEASUREMENTS - STANDARDS DEVELOPMENT-_-

Standard Reference Material and Reference Method for Asbestos in Air:

Project Description--.---_-

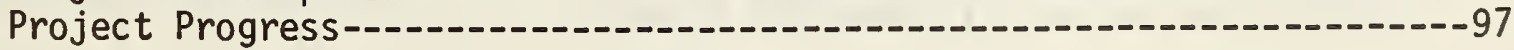

Standard Reference Materials (SRM's) for Toxic Organics in Air:

Project Description--

Project Progress---108

Acid Precipitation Quality Assurance Samples: Project Description---------111 Project Progress-----------112

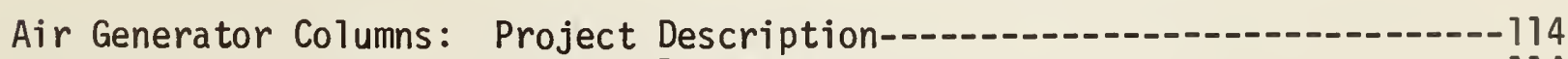
Project Progress---on 


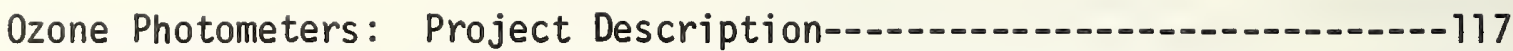

Project Progress---o-1

Energy-Related Gas Standards Development: Project Description--------120

Project Progress----------120

Energy-Related Particulate Standards Development:

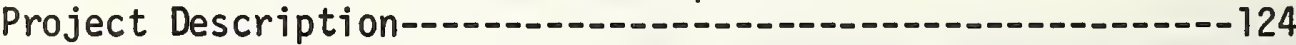

Project Progress---o-_-

AIR MEASUREMENT - METHODS DEVELOPMENT

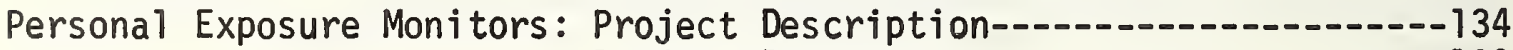

Project Progress--_-

Calibration and Measurement Technique Development for UV

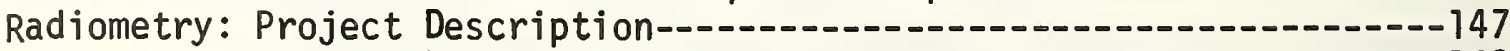

Project Progess---on

Energy-Related Gas Methods Development: Project Description----------151

Selected Technical Activities

Standards for Measurement of Atmospheric Carbon Dioxide----------152

Intercomparison of $\mathrm{O}_{3}, \mathrm{NO}$ and $\mathrm{NO}_{2}$ Measurement Standards by Gas

Phase Titration----_

Energy-Related Particulate Methods Development: Project Description-156

Selected Technical Activities

Particle Doppler Shift Spectrometer (PDSS)---_-_-157

Measurement of Ambient Sulfur Aerosols by a New Fast Response

Flame Photometric Instrument---_-

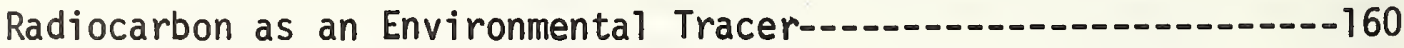

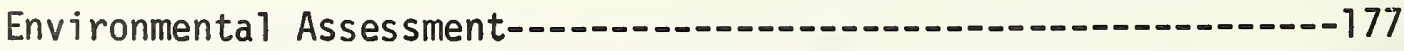

INTERACTIONS AND OUTPUTS

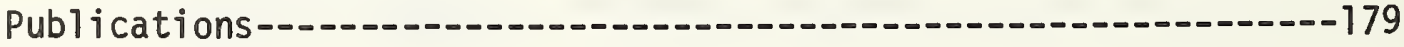


APPENDIX

NBS Organization Chart-10

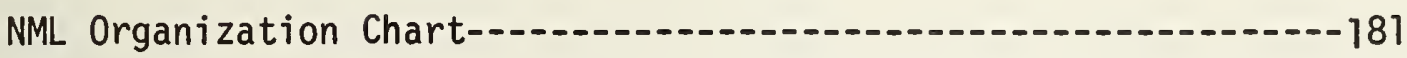

OEM Organization Chart-1-182

Functional Statement, OEM--183 



\section{DISCLAIMER}

"Certain commerical equipment, instruments, or materials are identified in this paper in order to adequately specify the experimental procedure. Such identification does not imply recommendation or endorsement by the National Bureau of Standards, nor does it imply that the materials or equipment identified are necessarily the best available for the purpose." 


\section{ABSTRACT}

This report summarizes the technical activities of the National Bureau of Standards' Environmental Measurements Program during Fiscal Year 1980. These activities fall into four program categories:

1. Water Measurements - Standards Development

2. Water Measurements - Methods Development

3. Air Measurements - Standards Development

4. Air Measurements - Methods Development

Key Words: Air; water; environmental measurements; methods; quality assurance; standards 


\section{INTRODUCTION}

This report summarizes the technical activities of the National Bureau of Standards' Environmental Measurements Program for the period October 1, 1979 to September 30, 1980. The Program is administered by the Office of Environmental Measurements (OEM) which is one of seven program offices in the National Measurement Laboratory concerned with the development and/or provision of various services in particular areas that rely upon a variety of disciplines located in NBS Centers. The technical work described in this report was performed by scientists located in three Centers - the Center for Analytical Chemistry, the Center for Radiation Research and the Center for Chemical Physics.

The primary goal of the NBS Environmental Measurements program is to provide standards of measurement to insure proper assessment of national environmental goals and to allow the attainment of these goals with the minimum of economic and energy penalty. For several years, the NBS Environmental Measurement Program was supported by direct Congressional appropriation. However, in fiscal year 1979, in response to an Office of Management and Budget (OMB) ruling, NBS activities in environmental measurements were required to be coordinated with and supported by the Environmental Protection Agency (EPA), the lead agency for achieving national environmental objectives. Therefore, the Environmental Measurements Program currently receives nearly $90 \%$ of its support from EPA and the majority of the technical activities summarized in this report are related to and in support of larger programmatic objectives of the of EPA Office of Monitoring and Technical Support.

The technical activties are described under four main headings: Water Measurements - Standards Development; Water Measurements - Methods Development; Air Measurements - Standards Development; Air Measurements Methods Development. An overview is given for each activity along with the staff members associated with that activity and selected highlights. A list of OEM publications for the year are listed at the end of this report.

A detailed Table of Contents has been provided which will permit the reader to find those activities of greatest interest. If more information about particular work is desired, the reader can address the individual scientists, c/o Office Environmental Measurements, National Bureau of Standards, B1dg. 220, Room A261, Washington, DC 20234 or contact this office by telephone (301)-921-3775. 



\section{WATER MEASUREMENTS - STANDARDS DEVELOPMENT}

\section{a. Overview}

The scope of the national program in water pollution control is mandated by recent Federal legislation and involves the efforts of municipal and state government agencies, industries, water suppliers and nearly every department in the Federal Government. Measurement forms the basis for nearly all decisions concerning water pollution. This task addresses the development of standards and Standard Reference Materials to assure accurate and compatible measurement throughout the water pollution measurement system and minimize the uncertainty associated with quantifying the extent of water pollution.

\section{Objective}

Provide measurement standards that are required to achieve accurate measurements of: (1) the concentration of chemical pollutants in water; (2) the chemical and physical properties of identified pollutants in water; and (3) the physical properties of water, such as velocity and flow. Water pollution standards developed in this task will provide an accurate, rational and equitable basis for the development and enforcement of water pollution regulations.

\section{Interactions}

Strong interactions are maintained with other units of the NBS community (Center for Analytical Chemistry, Office of Standard Reference Materials, Center for Radiation Research, Center for Thermodynamics and Molecular Science, Office of Standard Reference Data, Office of Recycled Materials, Center for Materials Science, Center for Mechanical Engineering and Process Technology, Center for Applied Mathematics, Office of Nuclear Waste Management), and with other Federal Agencies-(EPA, DOE, NOAA). The activities in this task also involve interactions with industry, state and local governments, research laboratories, universities, as well as with other organizations (e.g., Council on Environmental Quality, ASTM) engaged in related activities which impact the development of standards for water pollution measurements.

\section{Task - Justification}

The national effort in water pollution abatement involves municipal and state government agencies, industries, water suppliers and nearly every department of the Federal Government. In response to water pollution legislation (P.L. 92-500, P.L. 93-523), there has been an increase in water monitoring to determine progress toward national environmental goals, and to enumerate in quantitative terms regulations to achieve those goals. The national effort in water pollution abatement depends upon accurate and reliable measurement necessitating the need for accurate standards against which water pollution measurements can be 
compared. Without accurate measurements scientists cannot relate health effects to levels of water pollution correctly, engineers cannot assess the effectiveness of control techniques correctly, and regulatory agencies cannot relate levels of pollution effluents with water quality correctly nor can compliance with water pollution regulations be fairly and equitably tested.

b. List of Projects

Project Title

(1) Toxic Organics - Water

(2) Environmental Radioactivity Measurements

(3) Hazardous Waste Quality Assurance Samples

(4) Energy Related Water Standards (Organic and Inorganic)

(5) Radiological Pollutant Quality Assurance

* $P / T=$ Pass - Through Funding

$E / E=$ Energy $/$ Environment Funding

\section{Source of * \\ Funding}

$P / T$ - EPA

$P / T$ - EPA

$P / T$ - EPA

$E / E-E P A$

$E / E-E P A$ 
c. Project Description

(1) Standard Reference Material (SRM) for Organics in Water

Participating Staff: Center for Analytical Chemistry

Goal :

Assist EPA in achieving and maintaining quality assurance in the measurement of organic pollutants in water by providing standards against which organic water pollution measurements can be compared and measurement technology tested.

\section{Background:}

The size of the chemical industry, the number of compounds that exist, and their pervasiveness, all seem to make the regulatory task of protecting the aquatic environment particularly challenging. It has been estimated that $>70,000$ chemicals are currently in use. Although only a small number of these compounds are toxic, the risk to human health and the effect on the aquatic environment is/will be the focus of attention now and for several years to come. One of the largest classes of compounds is organics, which are used widely in industry as precursors in the manufacture of synthetics and as solvents in various commercial operations. To identify and measure the concentration of organics in water, EPA/CINC conducts monitoring programs for organic compounds.

Essential to a successful monitoring activity is availability of certified Standard Reference Materials (SRM) in the measurement range of interest. Such standards are needed for calibration of the measurement technique and in quality assurance activities necessary to determine the precision and accuracy of the monitoring data collected. Organic standard materials having certified concentrations of organics for use with water monitoring programs have not been available, because the analytical methodology needed to produce such standard materials had to be developed first. NBS has developed the capabilities which now enables it to respond to some of the organic standard needs (capability/methodologies to characterize and produce inorganic SRM's already existed for many years). This work plan for FY-80-81 addresses the development of organic SRM's for use by EPA/CINC in their monitoring and quality assurance programs.

Scope:

Utilizing the generator column concept, as well as other analytical methodologies, NBS will provide SRM's for the measurement of selected organic priority pollutants. 
Mi lestones:

1. Determine the solubilities of representative priority pollutants in order to assess the feasibility of generator column SRM's.

2. Assess the feasibility of preparing pesticide generator column SRM's for compounds to be identified by EMSL/CINC.

3. Complete certification of three generator columns (benzo(a)pyrene; benz(a)anthracene; anthracene)

4. Conduct a workshop at EPA/CINC, for participants selected by EPA/CINC, on the use/technique of generator column standards

5. Assess the feasibility of three pesticide generator columns to be selected by EPA/CINC from a previousiy proposed list

6. Complete the stability study for the halocarbons in water SRM

7. Prepare a SRM package for evaluating extraction efficiencies of PAH's from aqueous solution and conduct a "roundrobin" experiment with (a) a predetermined set of generator columns and (b) an acetonitrile solution of the 16 priority pollutant PAH's listed in EPA Interim Method 610.

8. Conduct a round-robin experiment with an acetonitrile solution of the 16 priority pollutant PAH's (see 7 above) plus 10-15 interferences. Issue Report

9. Procure and complete certification of indeno$(1,2,3-c d)$ pyrene generator column SRM

10. If the three pesticides selected and studied in 5 above are feasible for generator column SRM's, procure and complete certification

11. Complete certification of the halocarbon in Water SRM (based on results obtained in 6 above) 
Selected Technical Accomplishments:

1. Certification of Generator Columns for Polynuclear Aromatic Hydrocarbons in Water (SRM 1644)

J. M. Brown-Thomas, W. E. May, W. J. Sonnefeld, R. A. Velapoldi,

P. A. White

Saturated solutions of polynuclear aromatic hydrocarbons (PAH) can be produced by passing distilled water through a column packed with glass beads coated with the PAH compound of interest. The aqueous solubility of the compound is a well-defined thermodynamic quantity. Therefore, saturated aqueous solutions produced by this column technique ("generator column") may also be defined as standard solutions at a given temperature.

Generator columns for anthracene, benz(a)anthracene, and benzo(a)pyrene constitute SRM 1644. The columns were prepared commercially and have been certified by two independent analytical techniques. The first technique involves quantitative extraction of the PAH from the aqueous stream by an "extractor column" packed with $C_{18}$ bonded phase, use of an acetonitrile-water effluent to transfer components from the extractor column to an analytical $C_{18}$ column for separation of the analyte from non-analyte components, and detection of the analyte by absorbance at $254 \mathrm{~nm}$. The second technique uses a "standard addition" technique for "on stream" analysis. The effluent from the generator column is mixed with PAH standards dissolved in acetonitrile, and the resultant PAH in the mixed solvent determined by fluorescence. Exemplary data from the certification process are given in Table 1. One equation is derived from a compilation of similar data obtained from the two methods which will allow the user to interpolate the certified concentration between $10^{\circ} \mathrm{C}$ and $30^{\circ} \mathrm{C}$. 
Exemplary Results of Analysis Using Two Techniques for Generator Column Aqueous Effluents.

Temperature $\left({ }^{\circ} \mathrm{C}\right)$

30

25

20

15

Temperature

$\left({ }^{\circ} \mathrm{C}\right)$

30

25

20

15

Temperature

$\left({ }^{\circ} \mathrm{C}\right)$

30

25

20

15

10

\section{Anthracene}

Concentration (ppb)
Technique I

58.8

59.6

42.8

42.9

31.1

30.9

22.6

22.2

Benz(a)anthracene

Technique 1

12.3

13.0

8.86

9.32

6.37

6.67

4.58

4.78

Benzo(a)pyrene Technique I

2.28

2.26

1.62

1.60

1.14

1.14

0.80

0.81

0.57

0.58 
2. Determined Aqueous Solubiiities and Chromatographic Characteristics for Organic Compounds on the Priority Pollutant List

S. A. Wise, W. J. Bonnet

A knowledge of aqueous solubilities is the first step toward the development of aqueous organic SRM's utilizing the generator column technology. Since such data is sparse in the scientific literature, solubility measurements have been made on several organic compounds to date. The solubility data is displayed in table 2 along with an assessment of feasibility of generator column SRM's for priority pollutants.

3. Developed and Evaluated a Quenchofluorometric Detection System for Chlorinated Pesticides

B. F. Howe11, P. J. Logenbach, S. A. Wise

The quenching of fluorescence by pesticides has been observed, as noted in the literature by Lakowicz and Omann, who used this fact to study pesticide uptake into membranes [Science, 197, 465 (1977)]. This quenching ability of certain pesticides has been used to formulate a method for the selective detection of pesticides as they elute from an HPLC system.

The fluorescing probe used in the system developed was $\mathrm{N}$-ethy 1 carbazole. Initial fluorescence studies determined that $p, p^{\prime}-D D E$, lindane, endrin, $\mathrm{P}, \mathrm{p}^{\prime}-\mathrm{DDT}$, heptachlor, and $\alpha$-hexachlorocyclohexane quench the fluorescence of $\mathrm{N}$-ethyl carbazole in cyclohexane. In these studies the concentration of the pesticides was approximately $0.005 \mathrm{M}$ and the $\mathrm{N}$-ethy 1 carbazole, $3 \times 10^{6} \mathrm{M}$. The pesticide $p, p^{\prime}-D D E$ was found to be the most effective quencher of those studied, and a preliminary study showed its quenching to be linear from $0-200 \mathrm{ppm}$ in $1 \times 10^{-6} \mathrm{M}$ $\mathrm{N}$-ethyl carbazole.

In adapting this method to an HPLC detection scheme, the $\mathrm{N}$-ethy carbazole was dissolved directly in the mobile phase. The spectrometer was set to observe its steady background fluorescence. Injection of a pesticide sample was detected as a decrease in the fluorescence.

A reverse phase HPLC system using a $C_{18}$ column and a mobile phase of 85 percent $\left(9.93 \times 10^{-7} \mathrm{M}\right.$ N-ethyl carbazole in methanol), 15 percent $\mathrm{H}_{2} \mathrm{O}$ was found to separate endrin, $p, p^{\prime}-D D T$, and $p, p^{\prime}-D D E$. Injections on the order of $0.1 \mathrm{mg}$ of these pesticides gave good response in the fluorescence detection scheme, and could simultaneously be monitored using a UV detector at $254 \mathrm{~nm}$. It was found that the response observed by fluorescence detection did vary with the concentration of $\mathrm{N}$-ethyl carbazole. The peak height for equal injections of $p, p^{\prime}-D D E$ varied by a factor of $10^{3}$, when using $\mathrm{N}$-ethyl carbazole ranging in concentration from $1 \times 10^{-4} \mathrm{M}$ to $1 \times 10^{-8} \mathrm{M}$, with the largest peak being observed with the most concentrated $\mathrm{N}$-ethyl carbazole. 
The significance of retention data can be demonstrated by using two compounds in the Table 3 as examples, $p, p^{\prime}-$ DDD and heptachlor epoxide: the retention times shown under Condition I (i.e., 2.3 and 2.4 respectiveiy) for these two compounds indicate they could not be separated under Condition I, but could be efficientiy separated under condition II (i.e., retention times of 6.8 and 4.0 respectively).

With $\mathrm{N}$-ethyl carbazole present in the mobile phase, a constant fluorescence signal is obtained. The presence of a chlorinated pesticide quenches this fluorescence, giving a peak in the negative direction. Data for comparing the limits of detection for eight pesticides by "fluorescence quenching" and by UV $(254 \mathrm{~nm})$ is shown in Table 4. 
Table 2

Solubility Values for Priority Pollutants and "Generator Column SRM" Assessment

Hydrocarbons

1. acenaphthene

2. naphtalene

3. acenaphthylene

4. fluorene

5. fluoanthene

6. pyrene

7. anthracene

8. phenanthrene

9. benz(a)anthracene

10. chrysene

11. benzo(a)pyrene

12. 3,4-benzof luoranthene

13. 11,12-benzofluoranthene

14. dibenzo(ah)anthracene

15. indeno $(1,2,3-c d)$ pyrene

16. benzo(ghi)perylene

17. benzo $(j)$ fluoranthene

18. benzo(k)fluoranthene

19. benzene

20. toluene
Solubility (ppb)

31400
3900
1600
206
132
43
1000
9.8
2.2
1.2
1.5
3.2
$<0.1$
0.2
0.1
2.5
0.8
$1.8 \times 10_{5}^{6}$
$7.7 \times 10^{5}$

Phenols

1. phenol

2. pentachiorophenol

3. p-chloro-m-cresol

4. 2,4,6-trimethylphenol

5. 2-chiorophenol

6. 2,4-dimenthy 7 phenol

7. 2,4-dichlorophenol

8. 1,2-dichlorophenol

9. 2-nitrophenol

10. 4-nitrophenol

11. 2,4-dinitrophenol

12. 4,6-dinitrophenol

13. 4,6-dinitro-0-cresol

Suggestion

All too soluble for generator column SRM. Maybe aqueous solutions stable enough.

Suggested Generator Columns

\section{anthracene}

benz (a) anthracene

benz (a)pyrene

indeno $(1,2,3-c d)$ pyrene

Pesticides \& Metabolites

1. aldrin

2. dieldrin

3. chlordane

4. 4, 4'-DDT

5. $4,4^{\prime}-$ DDE

6. $4,4^{\prime}-D D D$

7. $\alpha$-endosolfan

8. $\beta$-endosulfan

9. endosulfan sulfate

10. endrin

11. endrin aldehyde

12. heptachlor

13. heptachlor epoxide

14. toxaphene
Because they cover the appropriate solubility range (i.e., $\sim 50-0.1 \mathrm{ppb}$ )
Solubility (ppb)

200,17
250
1850
1
1
20
530
280
--
260
---
---
350
---

Suggested Generator Column Standards

a)drin

dieldrin

4, 4'-DDD 
Table 2 (cont.)

Assessment of Priority Pollutant

\begin{tabular}{ll}
\multicolumn{1}{c}{ Benzenes } & Solubility (pp \\
1. benzene & $1.8 \times 10^{6}$ \\
2. chlorobenzene & $4.5 \times 10^{5}$ \\
3. 1,2-dichlorobenzene & $1.5 \times 10^{5}$ \\
4. 1,3-dichlorobenzene & $1.2 \times 10^{5}$ \\
5. 1,4-dichlorobenzene & \\
6. 1,2,4-trichlorobenzene \\
7. hexachlorobenzene \\
8. ethylbenzene
\end{tabular}

Suggested Generator Columns

All too soluble

PCB's Solubility (ppb)

1. Arochlor 1242

240

2. Arochlor 1254

3. Arochlor 1221

4. Arochlor 1232

5. Arochlor 1248

6. Arochlor 1260

7. Arochlor 1016

Suggested Generator Columns

None because mixed generator columns do not give saturated solutions.

\section{Nitrosamines}

$\mathrm{N}$-ni trosodime thy lamine

$\mathrm{N}$-nitrosodiphenylamine

$\mathrm{N}$-nitrosodi-n-propylamine

\section{Chloroalkyl ethers}

bis-(chloromethy 1$)$ ether

bis-(2-chloroethy 1) ether 2-chloroethylvinyl ether
Phthalate Esters

1. bis(2-ethylhexy1) phthalate

2. butyl benzyl phthalate

3. di-n-butyl phthalate

4. di-n-octyl phthalate

5. diethyl phthalate

6. dimethyl phthalate
Solubility (ppb)

$1.2 \times 10^{6}$

$1.1 \times 10^{4}$

$1.2 \times 10^{6}$

Suggested Generator Columns

All too soluble.

Miscellaneous

Solubility (ppb)

1. acrolein

2. acrylonitrile

3. benzidine

4. 2-chloronaph thalene

5. isophorone

6 . toluene

$7.3 \times 10^{5}$

It is NBS opinion that generator column SRMS could not be prepared for any of these compounds. 


\section{Halocarbons \\ (a11 too soluble)}

Literature

Solubility (ppb)

1. methylene chloride

2. methyl chloride

3. methyl bromide

4. bromoform

5. dichlorobromomethane

6. trichlorofluoromethane

7. dichlorodifluoromethane

8. chlorodibromomethane

9. hexachlorobutadiene

10. chloroethane

11. 1,2-dichloroethane

12. 1,1,1-trichloroethane

13. hexachloroethane

14. 1,1-dichloroethane

15. 1,1,2-trichloroethane

16. 1,1,2,2-tetrachloroethane

17. 1,1-dichloroethylene

18. 1,2-dichloroethylene

19. 1,2-dichloropropane

20. 1,2-dichloropropylene

21. vinyl chloride

22. hexachlorocyclopentadiene

23. $\alpha-B H C$

24. $B-B H C$

25. $\gamma-B H C$

26. $\triangle-B H C$

$1.8 \times 10^{6}$

$3.9 \times 10^{6}\left(3.7 \times 10^{6}\right.$ NBS value $)$ 
Table 3

Retention Data for Pesticides

Conditions used:

I. Mobile Phase: $85 \% \mathrm{MeOH} / \mathrm{H}_{2} \mathrm{O}$

Column: DuPont $\mathrm{C}_{18}$

II. Mobile Phase: Hexane

Column: Browniee $\mathrm{NH}_{2}$

\section{Retention Time, $\mathrm{cm}$}

\section{Conditions I}

$p, p^{\prime}-D D D$

heptachlor epoxide

dieldrin

endrin

heptachlor

$p, p^{\prime}-D D T$

$p, p^{\prime}-D D E$

aldrin
2.3

2.4

2.9

3.0

3.9

4.1

5.4

6.3
Conditions II

6.8

4.0

4.8

3.8

1.4

2.7

1.5

1.2 
Table 4

Comparison of Detection Limits for Eight Pesticides

Mobile Phase: $80 \%\left(1 \times 10^{-4} \mathrm{M} \mathrm{N}\right.$-ethyl carbazole in $\left.\mathrm{CH}_{3} \mathrm{CN}\right)$

Column: DuPont $\mathrm{C}_{78}$

$\frac{\text { Quenchofluorometric Detection }}{(\text { approx.) det'n limit, } \mu g}$

$p, p^{\prime}-D D D$

heptachlor epoxide

dieldrin

endrin

heptachlor

$p, p^{\prime}-D D T$

$p, p^{\prime}-D D E$

a)drin
1.4

4.8

8.9

4.0

8.1

.96

.060

6.7 $\frac{\text { UV Detection }}{\text { det'n limit, } \mu \mathrm{g}}$

.012

.16

.093

.039

.19

.015

.0007

.084 
4. Liquid Cinromatographic Retention Characteristics of Polycyclic Aromatic Hydrocarbons - S. A. Wise and W. J. Bonnett

The retention characteristics of over 80 polycyclic aromatic hydrocarbons (PAH) have been investigated on octadecylsilane $\left(C_{18}\right)$ and aminosilane $\left(\mathrm{NH}_{2}\right)$ high-performance liquid chromatographic (HPLC) column packing materials. These retention data are compiled in the form of retention indices similar to the Kovats retention index system used in gas chromatography.

Several different $C_{18}$ materials were investigated and were found to vary in selectivity, and therefore have different retention indices for some PAHs. These retention indices, al though not unique for all $\mathrm{C}_{18}$ and $\mathrm{NH}_{2}$ columns from different manufacturing processes, are useful for the design of HPLC schemes for the analysis of complex mixtures of PAHs. Such schemes have been employed for the determination of PAHs in shale oil, air particulates, and marine sediments. These retention data have also been correlated with the structure of the various PAHs to elucidate the mechanism of HPLC retention of PAHs on $\mathrm{C}_{18}$ and $\mathrm{NH}_{2}$ materials. In addition, many of the PAHs studied are unavailable commercially; and therefore, these data will be valuable to other laboratories that do not have these compounds.

5. Stability Study of Ha lohydrocarbons

F. Guenther

Solutions of seven halohydrocarbons at varying concentration levels were prepared and stored both at room temperature and freezer temperature. The stability of these solutions were checked after two months and again after five months. The analytical method used to determine the concentration level (i.e. stability) in the halohydrocarbons was pentane extraction $(25 \mathrm{~mL})$ of $100 \mathrm{~mL}$ water, followed by on column injection into a 20 meter SE-52 capillary column ( $1.0 \mu \mathrm{m}$ film thickness) with electron capture detection. This method was chosen because of its higher reproducibility in comparison to the purge and trap method and because of the short analysis time and resolution obtainable with a capillary column.

It was determined that a relative standard deviation of approximately $5 \%$ was obtainable. These data were obtained using three separate extractions of prepared aqueous halocarbon solutions. Bromotrichloromethane was used as the internal standard.

The purge and trap technique was also investigated, and showed a relative standard deviation in excess of $10 \%$. Our studies utilized the CDS 310 concentratorinstalled on a Varian $3700 \mathrm{GC}$. The analytical column was a $3^{\prime} 1 \%$ SP1000 on carbopack B.

Results of a five month stability study are shown in Table 5. The first column gives the gravimetric concentrations of the starting material, the second column is the results from the latest analysis and the last column gives the average concentrations over the last nine analyses.

The problem with chloroform has not yet been identified. It could be instability, inhomogeneity, or a measurement problem. Work is proceeding to identify the problem. The rest of the compounds show a slight 
Table 5

\section{Concentration (ppb)}

\begin{tabular}{|c|c|c|c|c|c|}
\hline \multirow{2}{*}{$\begin{array}{l}4 / 3 / 80 \\
76.2\end{array}$} & \multicolumn{3}{|c|}{$9 / 4 / 80$} & \multicolumn{2}{|c|}{$\begin{array}{r}\text { Avg. of } \\
9 \text { Runs ( } \pm \text { SD } \\
\end{array}$} \\
\hline & 56 & \pm 1 & & & --- \\
\hline 1.59 & 1.44 & \pm & .04 & 1.48 & \pm .05 \\
\hline 3.88 & 3.59 & \pm & .03 & 3.6 & \pm .1 \\
\hline 1.27 & 1.19 & \pm & .02 & 1.19 & \pm .03 \\
\hline 0.920 & 0.81 & \pm & .01 & 0.83 & \pm .06 \\
\hline 0.388 & 0.342 & \pm & .008 & 0.34 & \pm .04 \\
\hline 0.247 & 0.219 & & .008 & 0.22 & \\
\hline
\end{tabular}

Chloroform

Carbon tetrachloride

Bromodichloromethane

Dibromochloromethane

Trichloroethene

Tetrachloroethene

Tribromomethane

0.247

$0.219 \pm .008$

$0.22 \pm .02$ 
decline in concentration over the five months. This resulted from a new weighing technique, which corrected an earlier bias in the analytical scheme. The averages, however, show a relative standard deviation of 3 percent for the higher concentrations to 10 percent for the lower concentrations. This reflects the ampoule to ampoule homogeneity and the analytical reproducibility. No differences have been identified between the ampoules kept at room temperature'and those kept in the freezer.

A new set of ampoules were made up in September 1980, and will be analyzed for stability in the coming months. This will resolve the chloroform problem and the stability of the other compounds over the short run. Analysis of the original ampoules will also continue to resolve long term stability. An independent HPLC method of analysis is also currently being developed.

\section{Developed Alternate Proposal for Quality Assurance of PAH Mea- surement in Water}

Goal :

Assist EPA in achieving and maintaining quality assurance in the measurement of PAH in water by providing reference (rather than SRM's which by definition require more stringent characterization often resulting in longer certification/production periods) standards against which organics in water measurements can be compared.

Scope:

1) Utilizing the generator column concept, NBS will provide a Standard Reference Material package for evaluating extraction efficiencies of PAH from aqueous solution. We recommend that anthracene (45 ppb), benz(a)anthracene ( $10 \mathrm{ppb})$, benzo(a)pyrene (-1 ppb) and indeno(1,2,3-cd)pyrene $(\sim 0.2 \mathrm{ppb})$ be used to comprise this package.

2) NBS will also provide a reference standard for the chromatographic

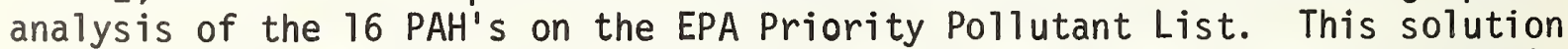
will be acetonitrile solution consisting of the 16 priority pollutant PAH's plus 10-15 other PAH's that commonly appear in extracts from aqueous effluents from oil shale processing and coal conversion plants.

3) NBS will develop a sequential HPLC analytical procedure with spectrofluorimetric detection for the analysis of the 16 priority pollutant $\mathrm{PAH}$ in the presence of other PAH interference such as those expected in real-world samples. The chromatographic procedure proposed in EPA Interim Method 610 allows for the separation of the 16 priority pollutant PAH's one from the other, in simple synthetic solutions containing a limited number of interferences. That method, however, will yield erroneous results when used for the analyses of more complex solutions similar to those that will be derived from aqueous effluents from energy conversion plants. For example on the Perkin Elmer column mandated by method 610: 
a) Several methyl chrysenes, and methyl benz(a)anthracenes, benzo(e)pyrene and perylene will all interfere with the analys is of benzo(b)fluoranthene.

b) 2-methyl anthracene will interfere with the analysis of fluoranthene.

c) 1-methyfluorene will interfere with the analysis of anthracene.

Milestones:

1) Conduct a "round-robin" experiment with (1) a predetermined set of generator columns and (2) an acetonitrile solution of the 16 priority pollutant PAH.

2) Conduct a round-robin experiment with an acetonitrile solution of the 16 priority pollutant PAH's plus 10-15 interferences.

3) Provide EMSL/CINC with a reference standard package consisting of four generator columns; anthracene, benz(a)anthracene, benza(a)pyrene, and indeno $(1,2,3-c d)$ pyrene and the organic solution of PAH's. 


\section{(2) Environmental Radioactivity Measurements}

Participating Staff: Center for Radiation Research - J. M. R. Hutchinson,

J. R. Noyce, K. G. Inn, F. J. Schima, M. J. Unterweger, L. L. Lucas,

D. D. Hoppes, B. J. Coursey

\section{Goa 1}

Assist EPA in achieving and maintaining an optimum radiological monitoring program to ensure quality assurance in the measurement of environmental radioactive pollutants by providing measurement methodology and radioactive SRM's and/or calibration standards.

\section{Background}

There is great public concern over the use of nuclear technologies because of possible associated radioactive contamination of the environment and Federal legislation (e.g. Clean Air Act, Clean Water Act, Safe Drinking Water Act) requires monitoring of radioactive substances in the environment. The assessment of regional and national effects of radionuclides on the environment depends upon accurate and reliable radionuclidic measurements.

The regulators of radioactivity at the national level (e.g. EPA, NRC) as well as state and local agencies have recognized the need for an effective National Radioactivity Measurement System. Essential to such a measurement system is the availability of certified standard materials, test samples/sources and calibration services. The NBS contributes to this measurement system by the production and distribution of environmental radioactivity SRM's and test samples, through tracecability programs with key laboratories and by participating with organizations concerned with radioactivity standards, methods, and data. The FY-1980 work plan addresses work agreed upon by EPA/Las Vegas and NBS.

\section{Scope}

The NBS wi11:

- Develop methods/techniques to produce environmental level radioactivity standards in variety of matrices-aqueous, solid, human tissue, soil

- Provide radioactive standards, test samples and/or calibration services needed to support the EPA/Las Vegas traceability program as well as overall radiological pollutant quality assurance 
Milestones

1. Continue traceability study with EMSL/Las Vegas.

$\sim$ one test/month

2. Develop standards for a TLD measurement acsurance program

Indeterminate

3. Evaluate monitoring methods for radium-228 and report.

$5 / 80$

4. Prepare solution standard of plutonium-240 for use as

a radiochemical tracer in environmental monitoring.

$7 / 80$

5. Develop natural matrix reference material for radio- activity environmental monitoring programs.

$9 / 80$

6. Complete construction of a unique alpha spectrometer system which will be used to measure low activity levels of alpha emitting radionuclides.

7. Develop mixed gamma-ray sources (SRM's) for the calibration of $\mathrm{Ge}(\mathrm{L} i \mathrm{i})$ spectrometers used in environmental monitoring.

$8 / 80$

8. Develop gaseous SRM's of Krypton-85 and Xenon-133 in special geometries which can be used for calibrating detectors used in monitoring reactor stack effluents.

$8 / 80$

9. Conduct international intercomparisons on iron-55, cobalt-57, cadmium-109, and barium-133 to improve the measurement capability of these radionuclides

10. Develop a mixed gamma-ray standard for calibration of detection system which use "Marinelli Beaker". geometry in monitoring programs. 
Six samples will be submitted by NBS to EPA for measurement. EPA will submit the results to NBS and, if the results agree, Certificates of Traceability will be issued. Six EPA standard samples will be submitted to NBS with the calibration values, and NBS will make measurements to cross-check the EPA values. If appropriate, certificates of Traceability will also be issued in these cases. If discrepancies are revealed, EPA equipment can be recalibrated, using the information on the traceability certificate, or their methods can be revised.

2. Develop Standards for TLD Program

Develop ${ }^{133}$ Xe in air mixtures at required levels of radioactivity concentration. Mixing procedures will be developed. A reference calibration will be performed with $\mathrm{Ge}(\mathrm{Li})$ spectrometers and the 2inch ionization chamber for the mixtures of Xenon immersion tank sampling ampoules.

3. Monitoring Evaluation

The availabie methods for monitoring will be reviewed, through a survey of the literature, and evaluated. A report summarizing this work with recommendations will be submitted to EPA.

4. Solution Standard of Pu-240

Pu-240 solutions standards will be proposed and calibrated by means of $\alpha$-particle counting. The preparation procedure includes examination by $\alpha$-particle and $\gamma$-ray counting for impurities, multiple dilutions and source preparation liquid scintillation, $0.8 \pi \alpha$ and $0.1 \pi \alpha$ and $2 \pi \alpha$ measurements, and preparation of certificates.

5. Natural-Matrix Standards

The collection, drying, grinding, sieving, bottling and initial homogeneity testing of the lung, liver, and Rocky Flats East and West samples will be accomplished this fiscal year.

6. Alpha-particle-Spectrometer System

The four chambers will be designed and constructed. The surfacebarrier detectors selected, and the vacuum systems completed so that the equipment will be operational by the middle of this fiscal year. 
7. Long-lived Mixed Gamma-Ray Standards

The radioactivity components will be ordered and checked for radioactive impurities by $\gamma$-ray spectrometry, dilutions will be made, sources prepared, calibrations performed using coincidence counting, and ionization-chamber measurements made.

8. Noble Gas Standards Suitable for Calibration of Air Sampling Charcoal Traps

Development wifl 33 proceed initially with tests of stability of adsorption of 133 with respect to typical air samplers and charcoal traps over time periods ranging from $10 \mathrm{hrs}$. to 10 days. Calibration procedures for measuring activity before and after exposure to the trap will be tested. This fjş̧al year goal is to determine the accuracy level of activity of ${ }^{3} \mathrm{Xe}$ that can be attained using these traps.

9. International Intercomparisons

Four intercomparisons are planned, Fe-55, Co-57, Cd-109, Ba-133. Radioactive materials are purchased, solutions are prepared and calibrations performed. The solutions are distributed to participating laboratories who return their measured values which are collated and compared. If the values disagree, proposals are considered for remedial action.

10. Marinelli-Beaker Calibrations

Marinelli-Beaker sources, prepared as described by an IEEE standard, will be submitted to NBS. A similar beaker, containing the same radionuclides in known amounts, will be prepared at NBS. The unknown will be calibrated by comparison with the NBS standard using a $G e(L i)$-spectrometer. 
1. Provided Services to EPA/Las Vegas Under the Measurement Traceability Program

Traceability (or reliability) of radioactivity measurements is required or recommended under various regulation, agreements, regulatory guides, licensing provision, written standards and recommended practices. simply stated, traceability to NBS is established when an outside laboratory (in this case EPA/LV Quality Assurance Branch) correctly measures test samples provided by NBS, or when NBS measures standard samples provided by an outside laboratory and the analytical results agree within the assigned calibration limits. Some activities under the EPA/NBS measurement traceability program are shown below:

- Reference Radionuclide samples sent to EPA/LV

- Phosphorous-32

- Scandium-46

- Promethium-147

- Cesium-134

- Nickel-63

- Cobalt-57

- Completed measurement of EPA/LV standards sent to NBS

- Chromium-51

- Evaluated results and completed ratios of results reported by EPA/LV to NBS on test samples

Radionuclide

- Scandium-46

- Cesium-134

- Americium-241

- Cobalt-58

- Plutonium-238

- Cobalt-57

- Nickel-63
QAD/NBS

0.995

0.996

0.995

0.961

1.000

1.027

1.044 
TABLE 6

Radioactivity Concentrations of 13 XE

\begin{tabular}{|c|c|c|c|c|c|}
\hline $\begin{array}{l}\text { Sample } \\
\text { Munber }\end{array}$ & $\begin{array}{l}\text { Time of } \\
\text { Sample } \\
\text { [hr:min] }\end{array}$ & $\begin{array}{c}\text { Elapsed } \\
\text { rime } \\
\text { [min] }\end{array}$ & $\begin{array}{c}\text { Radioactivity } \\
\text { Concentration } R(i) \\
{\left[\mathrm{s}^{-1} \mathrm{~cm}^{-3}\right]}\end{array}$ & $\begin{array}{c}\text { Ratio } \\
P(i) / R(1)\end{array}$ & $\begin{array}{c}\text { Ionizotion } \\
\text { Chamber." } \\
{\left[m_{i n}{ }^{-1}\right]}\end{array}$ \\
\hline$i$ & $10: 53$ & 0 & $9.67 \pm 0.26$ & $2.000 \pm 0.027$ & 32.2 \\
\hline$?$ & $11: 05$ & 12 & $8.96 \pm 0.27$ & $0.967 \pm 0.028$ & 12.5 \\
\hline 3 & $11: 46$ & 54 & $9.45 \pm 0.26$ & $0.977 \pm .026$ & 15.6 \\
\hline 4 & $13: 22$ & 149 & $8.27 \div 0.22$ & $0.855 \pm .022$ & 22.5 \\
\hline 5 & $13: 37$ & 164 & $7.80 \pm 0.15$ & $0.807 \pm .015$ & 22.6 \\
\hline - & $13: 40$ & 167 & \multicolumn{2}{|c|}{ GLOVE BOX OPEKED/FLUSHED } & $=$ \\
\hline - & $13: 49$ & 176 & - & - & 15.0 \\
\hline - & $14: 03$ & 190 & - & $\cdots$ & 13.7 \\
\hline - & $14: 33$ & 220 & -. & -. & 11.7 \\
\hline
\end{tabular}

*Background reading prior to ${ }^{133} \mathrm{xe}$ release was $0.1 \mathrm{~min}^{-1}$ 


\section{Evaluated Monitoring Methods for Radium-228 and Issued a Report}

The technical 1iterature from 1967 through May 1980 was reviewed for methods related to radium-228 assays in water. The methods were evaluated for their suitability as potential EPA reference methods for drinking water assays. It was suggested the present EPA reference method (Krieger, 1975) be retained but improved and a second method (McCurdy \& Mellar, 1979), which employs beta-gamma coincidence counting, be added. Included in the report is a table that lists the principal features of 17 methods for radium-228 assays.

\section{Developed a Sampling Procedure for Calibration of ${ }^{13}{ }^{3} \mathrm{Xe}$-Air Mixtures}

A sampling procedure has been devised to determine exposures to beta-particle emissions of TLD's immersed in ${ }^{13}{ }^{3} \mathrm{Xe}$-air mixtures. The procedure utilizes an NBS (almost-spherical) glass ampoule, with a vacuum-cup stopcock attached as a sample. A Ge(Li) detector system has been calibrated for $\gamma$-ray efficiency with this source configuration. ${ }^{133} \mathrm{Xe}$ was released into a glove box used as an immersion chamber and samples of the ${ }^{133} \mathrm{Xe}$ air mixtures were taken in the sample bottles.

\section{Procedure}

Several ampoules were evacuated, stopcocks closed and placed inside a glove box which had been prepared as an immersion chamber. A fan was placed inside the glove box to continously mix and move the ${ }^{13}{ }^{3} \mathrm{Xe}$ and air mixture. The sampling ampoules and the Mylar-window ionization chamber were sealed in the glove box. Approximately 115 microcuries of ${ }^{13}{ }^{3} \mathrm{Xe}$ gas were released inside the glove box, which was estimated to have a volume of 312 liters, giving a calculated initial radioactivity concentration of $13.6 \mathrm{~s}^{-1} \mathrm{~cm}^{-3}$ or 360 picocuries $/ \mathrm{cm}^{3}$. The ${ }^{133} \mathrm{Xe}$ and air were mixed for 15 minutes, at which time constant readings of the ionization chamber were observed. The glove box remained sealed for 240 minutes. At various times, five samples were open for about 5 seconds and then closed. As these labeled ampoules were filled with the ${ }^{133} \mathrm{Xe}-$ air mixture, the time and ionization chamber reading were recorded. The glove box was opened and flushed with room air through an $1100 \mathrm{~m}^{3} / \mathrm{min}$ hood to a stack.

The labeled ampoules subsequently were assayed by measuring the 81-keV gamma-ray-emission rate with a calibrated $\mathrm{Ge}(\mathrm{Li})$ detector system. Corrections were made for the ampoule-wall thickness. Activity was calculated using a value of 0.371 for the probability per decay for the $81-k e V$ gamma ray in ${ }^{133} \mathrm{Xe}$ decay.

Table 6 lists the radioactivity concentrations of ${ }^{133} \mathrm{Xe}$ in the glove-box air space at the time that each sampler was filled and resealed. Also the ratio of the radioactivity concentration at the time of sampling to the appropriately decayed radioactivity concentration of the first 
sample is given. Lastly, the reading, at the time of sampling, of the Mylar-window ionization chamber is listed. The errors shown in the Table are the random uncertainties due to counting. These range from 2 to 3 percent depending on the length of measurement time for each ampoule.

Inspection of the two right-hand columns of the table illustrate some surprising results. The ionization-chamber readings increased with immersion time while the radioactivity concentration decreased with immersion time. The first phenomenon is attributed to ${ }^{13}{ }^{3} \mathrm{Xe}$ contamination of the Mylar-window ionization chamber which is borne out by the slow rise in the ionization-chamber readings during immersion followed by a slow reduction in the readings after the ${ }^{13}{ }^{3} \mathrm{Xe}-\mathrm{air}$ atmosphere was removed. The ionization-chamber after 3 days was equal to the original background reading. The second phenomenon is attributed to the loss of ${ }^{13} \mathrm{Xe}$ inventory inside the glove box by either or both, leaks and adsorption on the rubber seals and gloves and the ionization chamber itself.

This work demonstrates a potential problem in the monitoring of ${ }^{133} \mathrm{Xe}$ in air. The ${ }^{133} \mathrm{Xe}$ concentration was measured as decreasing some $20 \%$ in 225 minutes while a portable monitor indicated an $85 \%$ increase over the same period. ${ }^{13}{ }^{3} \mathrm{Xe}$ distributions in small enclosures is not predictable.

4. Seven Natural Matrix Materials have been Dried, Ground, Homogenized and Bottled

See Table 7 for tabular status of the Natural Matrix Materials. Preliminary assays have also been performed on five of these materialsRocky Flats Soil E (RFSE), Rocky Flats Soil West (RFSW), Columbia River Sediment (CRS) and Gyttya. Table 8 shows the current status of assay for five materials. 


\section{Table 7}

Status of Environmentally Calibrated Material

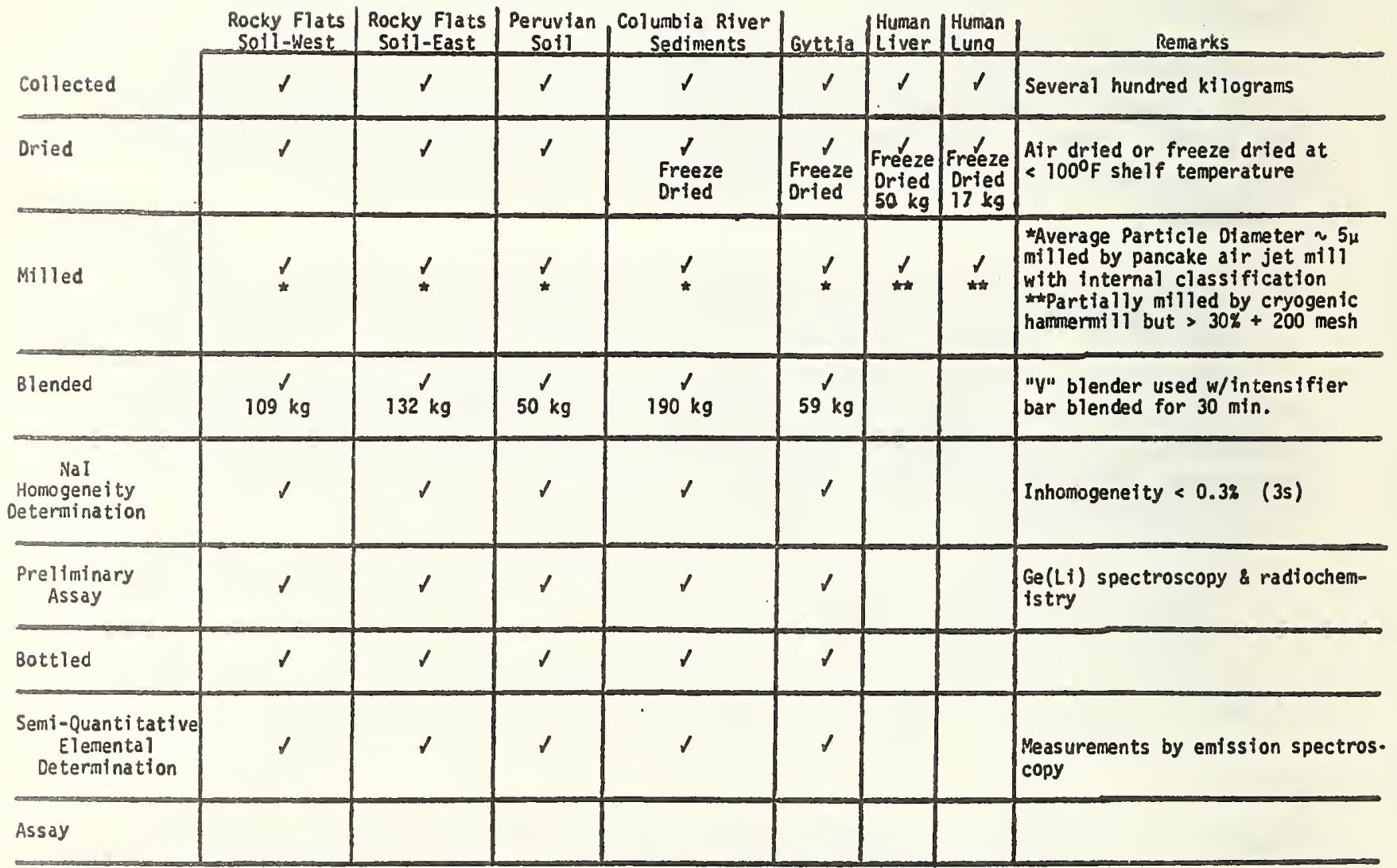




\section{Table 8}

STATES OF ASSAY OF NATURAL MATRIX STANDARDS (10/80)

Energy calibration

Efficiency calibration

Background

Chemistry

Source preparation

Blanks

Homogeneity

Count times

Activity levels

Data analysis

Equipment preparation

Equipment instability

Samples in the works

\begin{tabular}{|c|c|c|}
\hline$\alpha$ & $\beta$ & $\gamma$ \\
\hline+ & $N / A$ & + \\
\hline \multicolumn{3}{|l|}{$\checkmark$} \\
\hline$\checkmark$ & - & $N / A$ \\
\hline$\checkmark$ & - & $N / A$ \\
\hline$\checkmark$ & - & + \\
\hline \multirow[t]{2}{*}{$\checkmark$} & - & + \\
\hline & & $\checkmark$ \\
\hline 2 weeks & 2 months & I week \\
\hline $2 \mathrm{pCi} / \mathrm{sample}$ & pCi/sample & pCi/sample \\
\hline 1 & & $\checkmark$ \\
\hline$\checkmark$ & $\checkmark$ & + \\
\hline$\checkmark$ & $\checkmark<1 \%$ & $+<0.3 \%$ \\
\hline RFSE & & $\begin{array}{l}\text { RFSE } \\
\text { RFSW } \\
\text { CRS } \\
\text { GYTTJA }\end{array}$ \\
\hline
\end{tabular}

$+=$ completed work

$\checkmark=$ partially completed work

- = work initiated

blank space $=$ work yet to be initiated 


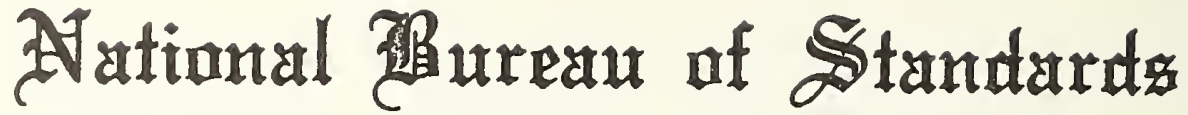

\author{
Report of Traceability Measurement by
}

QUALITY ASSURANCE DIVISION

U.S. ENVIRONMENTAL PROTECTION AGENCY

LAS VEGAS, NEVADA

\section{on an NBS Reference Sample of Undisclosed Value of} CESIUM-134

This is to report that on the basis of nine independent measurements on a solution of cesium-134 with a known, but undisclosed, radioactivity concentration on January 1, 1980, of

$$
\star 1.426 \times 10^{4} \mathrm{~s}^{-1} \mathrm{~g}^{-1} \pm 1.7 \% * \text {, }
$$

the Quality Assurance Division (QAD) reported a value of

$$
1.42 \times 10^{4} \mathrm{~s}^{-1} \mathrm{~g}^{-1} \pm 3.0 \%
$$

on January 1, 1980.

The uncertainty in the QAD value, 3.0 percent, is the linear sum of 2.0 percent, which is half the 99-percent confidence interval of the mean of the $\beta-\gamma$ coincidence-counting determinations, and 1.0 percent, which is the linear sum of the estimated upper limits of conceivable systematic errors.

The QAD value differs from the NBS value by 0.4 percent. This result demonstrates the traceability of QAD to NBS, for this measurement, to within five percent as specified in the appendix, "Traceability Studies", of the EPA-NBS interagency agreement of Apri1 1976.

The attached sheet provides supplemental information about this cesium-134 reference sample.

For the Director,

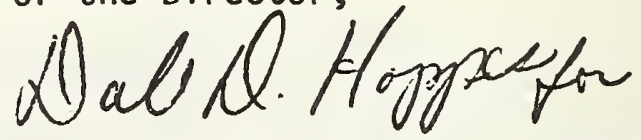

W.B. Mann, Principal Scientist Radioactivity Group Center for Radiation Research 


\section{SUPPLEMENTAL INFORMATION \\ ABOUT \\ CESIUM-134 REFERENCE SAMPLE \\ OF}

JANUARY 1980

This reference sample consisted of cesium-134 in 4.308 grams of carrier solution in a flame-sealed, borosilicate-glass ampoule. The solution contained 40 micrograms of stable cesium per gram of 1.9 molar hydrochloric acid. The reference sample was prepared by a gravimetric dilution of a master solution.

The radioactivity concentration of the master solution was measured using the National Bureau of Standards pressurized " $4 \pi " \gamma$ ionization chamber that had previously been calibrated with a cesium-134 standard in terms of a radium-226 reference source. A half life of $2.062 \pm 0.005$ years was used to calculate the cesium-134 decay from the date of calibration to the date of the QAD value (NCRP Report No. 58, App. A, National Council on Radiation Protection and Measurements, Washington, 1978).

The uncertainty in the cesium-134 radioactivity concentration of this reference source, 1.7 percent, is the linear sum of 0.43 percent, which is the 99 -percent confidence limit of the mean of ten ionization-chamber measurements, and 1.27 percent, which is the linear sum of the estimated upper limits of conceivable systematic errors. The estimated upper limits are $1.1_{4}$ percent in the ionization-chamber calibration, 0.03 percent in the computation of decay, and 0.1 percent in the weighings associated with the preparation of the reference source.

The master solution was examined for gamma-ray-emitting impurities with a Ge(Li)spectrometer system over the energy region of 90 to $1900 \mathrm{keV}$, and no impurities were observed. The detection limits for impurity photons can be expressed as a percentage of the emission rate of the 604-keV gamma ray emitted in the decay of cesium-134. The limits are 0.1 percent for photons with energies less than $790 \mathrm{keV}$ and 0.01 percent for photons with energies greater than $807 \mathrm{keV}$, provided that the impurity photons are separated by $5 \mathrm{keV}$ or more in energy from photons of equal or greater intensity emitted in the decay of cesium-134. 


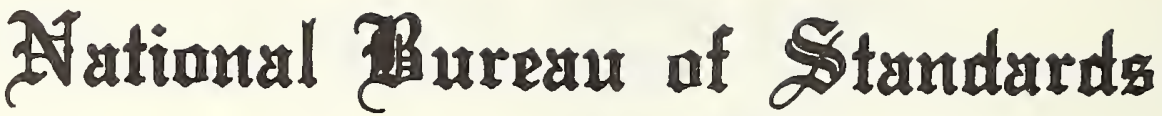

\author{
Report of Traceability Measurement by \\ Radiological and Environmental Sciences Laboratory \\ U.S. Department of Energy \\ Idaho Falls, Idaho
}

\section{on an NBS Reference Sample of Undisclosed Value of}

\section{SCANDIUM-46}

This is to report that on the basis of 12 independent measurements on a solution of scandium-46 with a known, but undisclosed, 889.25-keV gamma-ray emission rate per gram at 1400 EST Apri1 1, 1980, of

$$
\star 1.348 \times 10^{4} \mathrm{rs}^{-1} \mathrm{~g}^{-1} \pm 0.94 \% \text {, }
$$

the Radiological and Environmental Sciences Laboratory (RESL) reported a value of

$$
1.39 \times 10^{4} \mathrm{rs}^{-1} \mathrm{~g}^{-1} \pm 4.5 \%
$$

at 1400 EST Apri1 1, 1980.

The uncertainty in the RESL value, 4.5 percent, is the linear sum of 0.9 percent, which is half the 99-percent confidence interval of the mean of the germanium-spectrometer measurements, and 3.6 percent, which is the linear sum of the estimated upper limits of conceivable systematic errors.

The RESL value differs from the NBS value by 3.1 percent. This result demonstrates the traceability of RESL to NBS, for this measurement, to within five percent as specified in the document "Testing of NRC Reference Laboratory" of April 1976, as amended.

The attached sheet provides supplemental information about this scandium-46 reference sample.

For the Director,

W.B. Mann, Principal Scientist Radioactivity Group

Center for Radiation Research 


\author{
SUPPLEMENTAL INFORMATION \\ ABOUT \\ SCANDIUM-46 REFERENCE SAMPLE \\ OF \\ JANUARY 1980
}

This reference sample consisted of scandium-46 in 4.929 grams of carrier solution in a flame-sealed, borosilicate-glass ampoule. The solution contained 24 micrograms of stable scandium per gram of 1 M hydrochloric acid.

The sample was a portion of a solution whose radioactivity concentration was measured in the NBS $4 \pi \beta(L S)-\gamma$ anticoincidence system using the efficiency-extrapolation method. A half life of 83.830 .02 days was used to calculate the scandium-46 decay from the time of calibration to the time of the RESL value (NCRP Report No. 58, App. A, National Council on Radiation Protection and Measurements, Washington, 1978). The 889.25-keV gamma-ray emission-rate per gram is essentially equal to the radioactivity concentration $(\mathrm{Pr}=0.999840 \pm 0.000010$, ibid.).

The uncertainty in the $889.25-\mathrm{keV}$ gamma-ray emission-rate per gram, 0.94 percent, is the linear sum of 0.66 percent, which is half the 99-percent confidence interval of the mean for six independent calibration measurements, and 0.28 percent, which is the linear sum of the estimated upper limits of conceivable systematic errors. The estimated upper limits of the systematic errors are 0.1 percent in the weighings of the calibration sources, $0.1_{5}$ percent in the $4 \pi \beta(L S)-\gamma$ measurements, and 0.03 percent in the calculation for decay.

The scandium-46 was examined for gamma-ray-emitting impurities with a Ge(Li)-spectrometer system over the energy region of 90 to $1900 \mathrm{keV}$, and no impurities were observed. The detection limits for impurity photons can be expressed as a percentage of the emission rate of the $1120.5-\mathrm{keV}$ gamma ray emitted in the decay of scandium- 46 . The limits are 0.1 percent for photons with energies less than $1115 \mathrm{keV}$ (provided that the impurity photons are separated by more than $5 \mathrm{keV}$ in energy from the 889.25-keV photon emitted in the decay of scandium-46), and 0.01 percent for photons with energies greater than $1126 \mathrm{keV}$. 


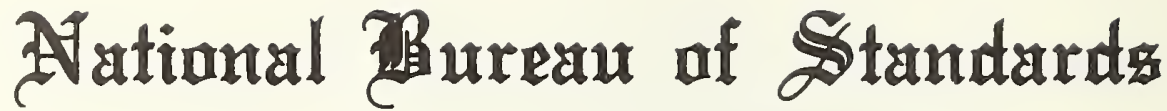

\section{Report of Traceability Measurement by}

Quality Assurance Division

U.S. Environmental Protection Agency

Las Vegas, Nevada

\section{on an NBS Reference Sample of Undisclosed Value of}

\author{
AMERICIUM-241
}

This is to report that on the basis of eight measurements with a $2 \pi$ gas-flow proportional counter on point sources prepared from a solution of americium-241 having a known, but undisclosed, radioactivity concentration on April 22, 1980 of

$$
\star 913.9 \mathrm{~s}^{-1} \mathrm{~g}^{-1} \pm 1.3 \% * \text {, }
$$

the Quality Assurance Division (QAD) reported a value of

$$
909 \mathrm{~s}^{-1} \mathrm{~g}^{-1} \pm 3.4 \%
$$

on Apri1 22, 1980.

The uncertainty in the QAP value, 3.4 percent, is the linear sum of 1.9 percent, which is half the 99-percent confidence interval of the mean of the proportional-counter measurements, and 1.5 percent, which is the linear sum of the estimated upper limits of conceivable systematic errors.

The QAD value differed from the NBS value by 0.5 percent. This result demonstrates traceability of QAD to NBS, for this measurement, to within five percent as specified in the appendix Traceability Studies of the EPA-NBS interagency agreement of April 1976, as amended.

The attached sheet provides supplemental information about this americium-241 reference source.

For the Director,

$$
\text { OMrdub hiu sa }
$$

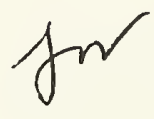

W. B. Mann, Principal Scientist

Radioactivity Group

Center for Radiation Research

June 12,1980 


\section{SUPPLEMENTAL INFORMATION}

about

A NBS REFERENCE SAMPLE OF

AMERICIUM-241

This reference sample consisted of americium-241 in $3.521 \mathrm{~g}$ of approximately 5 M nitric acid in a flame-sealed, borosilicate-glass ampoule, and was a portion of a master solution which was calibrated by liquid-scintillation counting of five sources that had been quantitatively prepared from the master solution.

The master solution was examined for photon-emitting impurities with a calibrated germanium spectrometer system and none was found. The detection limit for impurity photons may be expressed as a percentage of the emission rate of the $60-\mathrm{keV}$ gamma ray emitted in the decay of americium-241. The limit is 0.01 percent for photons with energies greater than $65 \mathrm{keV}$, provided that the impurity photons are separated in energy by $5 \mathrm{keV}$ or more from photons of equal or greater intensity emitted in the decay of americium-241.

The uncertainty in the radioactivity concentration of the reference sample, 1.3 percent, is the linear sum of 0.6 percent, which is half the 99-percent confidence interval of the mean of the liquid-scintillation measurements, and 0.7 percent, which is the linear sum of the estimated upper limits of conceivable systematic errors. The estimated upper limits are: 0.4 percent in the wall-absorption effects of the liquid-scintillation vials, 0.1 percent in the timing of the measurements, 0.1 percent for dead time during the measurements, and 0.1 percent in the preparation of the counting sources, including uncertainties in the weighings.

A half life of $433 \pm 2$ y (D. C. Kocher, ORNL/NUREG/TM-102, August 1977) was used in the calculation for decay from the date of calibration by NBS (May 23, 1979) to the date of the measurements by QAD (April 22, 1980). 


\title{
REPORT OF CALIBRATION
}

\author{
on one solution of \\ CHROMIUM-51 \\ submitted by \\ Quality Assurance Division \\ U.S. Environmental Protection Agency \\ Las Vegas, Nevada
}

One flame-sealed ampoule, said to contain chromium-51 in aqueous solution. was submitted for calibration as part of the Measurement Traceability Program for fiscal year 1980 between the National Bureau of Standards (NBS) and the Environmental Protection Agency (EPA).

The ampoule was opened and the solution gravimetrically transferred to an NBS standard ampoule. Several drops of water were added in order to make a total mass of five grams, and the ampoule was then flame sealed.

The gamma-ray-emission rate of the solution at $320 \mathrm{keV}$ was measured, on five different dates over a three-week period, with the ampoule positioned $26 \mathrm{~cm}$ from a $\mathrm{Ge}(\mathrm{Li})$ detector with a known detection efficiency. Using a gamma-ray probability per decay of $0.0980 \pm 0.0003$ (a), the radioactivity concentration of chromium-51 in the undiluted EPA solution was

$$
* 9.14 \times 10^{3} \mathrm{~s}^{-1} \mathrm{~g}^{-1} \pm 5.5 \% *
$$

at 0700 EST December 13, 1979.

The uncertainty in the NBS value, 5.5 percent, is the linear sum of 3.0 percent, which is half the 99-percent confidence interval of the mean of the five gamma-ray emission-rate measurements, and 2.5 percent, which is the linear sum of the estimated upper limits of conceivable systematic errors. These estimated upper limits are: 1.0 percent in the calibration of the germaniumdetector efficiency at $320 \mathrm{keV}$ with a point source; 0.5 percent in the ratio of detection efficiency for an ampoule source to that for a point source; 0.3 percent in the positioning of ampoules for measurement and differences in geometry among ampoules; 0.2 percent for changes in detector efficiency over the period of the measurements; 0.3 percent in the gamma-ray probability per decay; and 0.2 percent in the calculations for decay, based on a half life of $27.704 \pm 0.004 d(b)$. 
The EPA reported a radioactivity concentration of chromium-51 for this solution of

$$
9.00 \times 10^{3} \mathrm{~s}^{-1} \mathrm{~g}^{-1} \pm 9.8 \%
$$

at 0700 EST December 13, 1979. The uncertainty in the EPA value, 9.8 percent, is the linear sum of 4.6 percent, which is half the 99-percent confidence interval of the mean of the germanium-spectrometer measurements on nine samples, and 5.2 percent, which is the linear sum of the estimated upper limits of known systematic errors.

The EPA value differs from the NBS value by 1.5 percent. This result demonstrates the traceability of EPA to NBS, for this measurement, to within five percent as stated in the appendix, Traceability Studies, of the EPA-NBS interagency agreement of Apri1 1976, as amended.

For the Director,

$$
\begin{aligned}
& \text { Mald Hetichic.... } \\
& \text { for w. B. Mann, Principal Scientist } \\
& \text { Radioactivity Group } \\
& \text { Center for Radiation Research }
\end{aligned}
$$

June 12,1980
(a) NBS value.
(b) NCRP Report No. 58, National Council on Radiation Protection and Measure- ments, Washington, D.C. (1978). 
(3) Hazardous Waste: Sludge Characterization and Fly Ash Characterization

Participants: Center for Analytical Chemistry - R. W. Burke, B. I. Diamonds tone

\section{Background}

With the passage of the Resource Conservation and Recovery Act in 1976, Congress mandated that EPA establish a set of regulations by Apri1 1978 regarding the identificaiton, transportation, and disposal of hazardous waste materials. EPA subsequently defined eight characteristics for waste materials, any one of which would classify a waste material as hazardous. One of these characteristics, EP toxicity, EPA has chosen to relate to the amounts of certain priority inorganic and organic pollutants which are leached from the wastes under a set of empirical extraction conditions.

Early in FY80, NBS (through the Office of Recycled Materials) entered into an interagency agreement with EPA to evaluate the several extraction procedures that were being considered as acceptable tests for the identification of hazardous wastes. A copy of this agreement (EPA 79D-X0810) can be found in the Appendix, this report (pp. 257). A detailed work statement for this project which was submitted to (and accepted by) EPA/Las Vegas is given in (C) of this section. The resources (\$110K) provided to the NBS Office of Environmental Measurement through the EPA Pass-Through program were in support of the objectives and milestones of this large hazardous was te effort. 
Office of Recycled Materials/Center for Aralytical Chemistry to EPA/Las Vegas)

Task 1: Initiate and complete studies on a representative "hazardous" fly ash as provided or recommended by EPA. These studies will result in the development and issuance of a quality assurance standard for use in EPA's intercomparison and laboratory accreditation programs. The outputs of this task are:

A. Collect, blend, sieve, and homogenize a minimum of $250 \mathrm{~kg}$ of the chosen fly ash.

B. Establish the chemical composition of this fly ash by using existing NBS standards and comparative instrumental techniques.

C: Determine the extraction and analysis variabilities on the leachates derived from this fly ash using the proposed EP and the extractor built under the FY 72 EPA project.

D. Develop reference leachate data using the EP for the eight priority inorganic pollutants, i.e. As, $\mathrm{Ba}, \mathrm{Cd}, \mathrm{Cr}, \mathrm{Pb}, \mathrm{Hg}$, $\mathrm{Se}$, and $\mathrm{Ag}$.

E. Package, label and deliver to EPA 500 units, each containing 105 grams, of the quality assurance fly ash standard.

Task II: Initiate studies on a sludge or sludge-like material as chosen in concert with EPA for possible future (FY 81 ) issuance as a more complex type of quality assurance standard. The outputs of this task include:

A. Obtain profile leachate data for priority inorganic on 3-5 candidate sludges recommended or provided by EPA.

*B. Survey the EP leachates obtained in step A for selected organics on the priority pollutant list, i.e. chlorinated compounds, phenols, polynuclear aromatics, etc. (Level of effort-- $\$ 30 \mathrm{~K}$ )

C. Study sampling, packaging, and storing techniques on the most promising sludge.

D. If step $C$ is successful, carry out detailed stability and homogeneity studies on $200-400 \mathrm{~kg}$ quantity of the selected sludge.

E. Establish the inorganic chemical composition of this sludge by use of comparative instrumental techniques and available NBS standards.

F. Obtain leachate data for the priority inorganic pollutants using the proposed EP and the extractor built under the FY 7.9. EPA project. 
Project costs: The above tasks are listed in priority order and need to be carried in that order. The anticipated costs for each task is as follows:

$$
\begin{aligned}
& \text { Task I } \$ 75,000 \\
& \begin{aligned}
\text { Task II } & 210,000 \\
\text { Totar } & \$ 285,000
\end{aligned}
\end{aligned}
$$

* Sub-task B, Task II, will be accomplished by the Organic Analytical Research Division, depending upon analytical committment and the availability of the indicated funds. All other sub-tasks will be accomplished by the Inorganic Analytical Research Division. 
1. Characterization of Potentially Hazardous Waste-Development of Rotary Extractor and Quality Assurance Standards

In order for NBS to fulfill its obligation to EPA, it was necessary to develop a procedure which would reproducibly extract the leachable priority pollutants from the waste material. Although a number of extraction techniques are presently in use, the results of various interlaboratory studies indicated that variations in concentrations of orders of magnitude for the priority pollutants are not uncommon. Although this can be a result of poor analytical technique and/or sample inhomogeneity, the possibility of poor extractor designs must also be considered. In fact, data presented at a number of ASTM meetings in which several members of NBS staff participated, clearly indicated that the reproducibility of the extraction procedures was extremely poor. Thus, in order for meaningful measurements to be made on an interlaboratory basis, an improved extractor design was of paramount importance.

Based on our interpretation of the EPA Federal Guidelines, a model extractor was designed which allowed four samples to be run simultaneously in 2-liter containers. The sample containers were tumbled endover-end at 29 rpm permitting intimate contact between the waste and the extracting solutions. Although extensive testing of this rotary extractor at NBS is incomplete, EPA has chosen to include this design as an acceptable model in the latest Federal guidelines. Presently, studies are in progress to evaluate the efficiency and abrasion characteristics of this design on selected wastes. An illustration of this rotating extractor is shown in figure 1 .

Currently, NBS is in the process of providing to EPA a number of units of representative waste material which will be a fly ash that is totally characterized for chemical composition using NBS standards. Information will also be provided on the concentrations of the priority pollutants which can be leached from the material under specific extraction conditions. In addition, NBS is initiating studies on some sludge materials to be used as more complex quality assurance standards. Work in this area is expected to continue in FY81. 


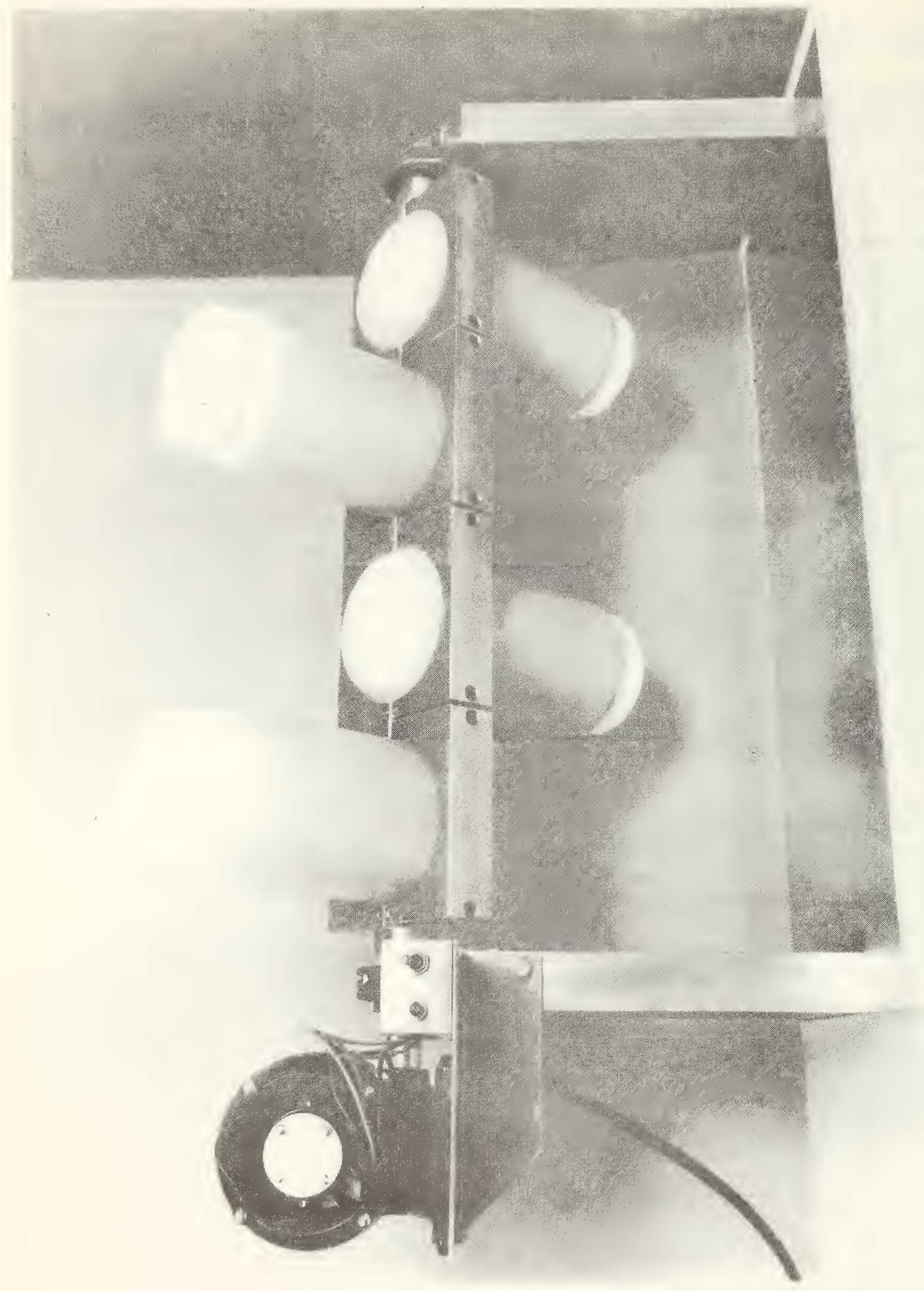


(4) Energy-Related Organic and Inorganic Water Standards Development Participating Staff: Center for Analytical Chemistry

\section{Background}

In order to assess the total effects of energy development upon our environment, an effective multi-media monitoring and measurement assurance system must be maintained. Development of a compatible data base to determine the source, transport, and fate of environmental pollutants is a necessity if rational and logical decisions are to prevail in planning the types, pattern, and magnitude of energy developments which are environmental7y acceptable. Since the western states are already or will be seriously impacted by present or projected energy-related development activities, the Western Energy/Environment Monitoring Study (WEEMS) was initiated to provide an accurate multi-media data base keyed to air and water pollutants and surface effects associated with projected western energy resource developments.

\section{Scope}

The activities in this project focus on the development of energy related water pollutant SRM's for conventional fossil fuel mining and combustion and SRM's for advanced fossil fuel technologies. The SRM's developed in this project will be provided to EMSL/ Cincinnati in support of the WEEMS project as well as in support of EPA quality assurance programs. Representative milestones for the standards development of the EPA/NBS E/E Accomplishment Plan for FY-1980 included:

- Development of SRM's or reference standards

(characterized for organics and inorganics) for measuring and monitoring water quality of the western water resources with particular emphasis on the Northern Great Plains area and the San Juan Basin.

- $\quad$ Assessment/feasibility of producing specific SRM's/reference standards for calibration of organics in water measurement methods for pollutants associated with existing energy technologies.

- Development of SRM's for use in methodology assessment, method calibration, and monitoring of water pollutants associated with emerging technologies. Included in this milestone is the need for development of environmental measurement methods capable of measuring trace toxic substances in complex matrices created by new energy technologies to support the establishment of effective monitoring systems. 
1. First Natural Matrix Trace Organic SRM Issued for Shale 0il (SRM 1580)

J.M. Brown-Thomas, S.N. Chesler, F.R. Guenther, H.S. Hertz, L.R. Hilpert, P.L. Konash, W.E.May, R.M. Parris, K.L. Richie, S.A. Wise

The Center for Analytical Chemistry have in the past conducted several collaborative studies aimed at assessing the accuracy of data obtained from trace organic analytical methodologies. The results of a recent study involving the determination of specified phenols, polynuclear aromatic hydrocarbons, and $\mathrm{N}$-heterocyclics in a shale oil sample by several laboratories revealed large systematic biases among laboratories. One method for enhancing the accuracy of analytical measurements is the use of suitable quality assurance standards or Standard Reference Materials (SRM's). However, until now, SRM's for environmental trace organic analyses were nonexistent due to the lack of analytical methodologies necessary for certification.

Over the past few years, analytical methodologies have been developed and used to certify the first natural matrix SRM for trace-level organic constituents, Organics in Shale 0i1, SRM 1580. The quantitation of individual compounds in the complex shale oil matrix necessitated the use of multi-dimensional chromatographic procedures, i.e., gas or liquid chromatography on two columns of different selectivity and/or extremely selective detection methods.

Three different methods of sample preparation were used prior to analysis: simple dilution of the shale oil with methylene chloride (or other suitable solvent); acid/base extraction to isolate acidic, basic, and neutral components; and a high-performance liquid chromatographic fractionation. Two of the following techniques were employed to determine the certified values for the organic compounds in shale oil: capillary gas chromatography (GC), gas chromatography/mass spectrometry (GC/PS) with single ion (monitoring) for selective detection, and high performance liquid chromatography (HPLC) with selective fluorescence detection. All GC/MS analyses used the standard addition method for quantitation. The GC and HPLC analyses employed either internal standard, external standard, or standard addition methods. The excellent comparability of the values determined by these different analytical methods is illustrated in Table 9. Table 101 ists values for other compounds determined by only one technique. These values will appear on the SRM certificate for information only. The methods developed for the certification of this SRM are applicable to the determination of similar compounds in other complex natural matrices. Standard Reference Material 1580, Organics in Shale 0i1, is intended primarily for evaulating the reliability of analytical methods used for the determination of trace level polynuclear aromatic hydrocarbons, phenols, and nitrogen heterocyclic compounds in shale oil, coal-derived liquids, or petroleum products. In addition, this SRM may be used as a surrogate standard for any complex environmental extract containing classes of compounds for which the SRM is certified. 


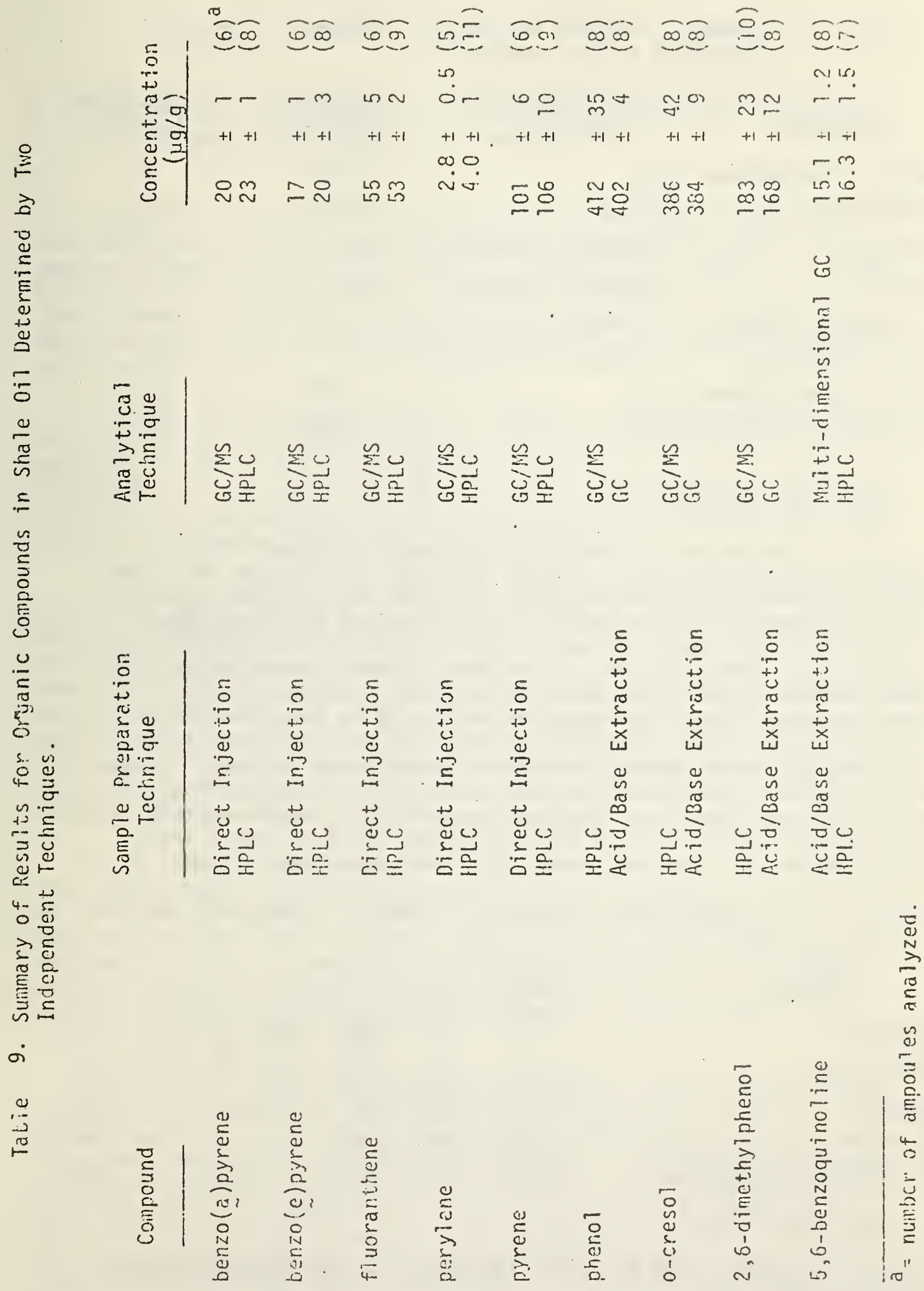


Table 10. Summary of Results for Organic Compounds in Shale 011 Determined by One Technique.

\begin{tabular}{|c|c|c|c|}
\hline Compound & $\begin{array}{c}\text { Sample Preparation } \\
\text { Technique } \\
\end{array}$ & $\begin{array}{l}\text { Analytical } \\
\text { Technique } \\
\end{array}$ & $\begin{array}{c}\text { Concentration } \\
(\mu \mathrm{g} / \mathrm{g})\end{array}$ \\
\hline benzo(k)fluoranthene & HPLC & HPLC & 13 \\
\hline phenanthridine & HPLC & HPLC & 45 \\
\hline m-cresol & Acid/Base Extraction & GC & 330 \\
\hline p-cresol & Acid/Base Extraction & GC & 270 \\
\hline 2,4-dimenthy 1 phenol & Acid/Base Extraction & GC & 380 \\
\hline 2,5-dimethylphenol & Acid/Base Extraction & GC & 320 \\
\hline $2,5,6$-trimethyl phenol & HPLC & $\mathrm{GC} / \mathrm{MS}$ & 360 \\
\hline $2,4,6$-trimethylphenol & HPLC & GC/MS & 120 \\
\hline
\end{tabular}

a. Shale 0il Round Robin:

Many laboratories are currentiy involved in the quantitative analysis of individual toxic organic compounds in al ternate fuels and energy effluents. For many of these environmental analyses there is little knowledge of the comparability of data from different laboratories, and in most cases probably little knowledge of intralaboratory precision. To gain insight on this matter aliquots of the shale oil sample were sent to six laboratories currently involved in characterizing alternate fuels. The labs were reques ted to determine the concentrations of selected compounds in the shale oil. The results of this limited inter-laboratory exercise are presented in Table 11. The scatter of the results indicates the variability of state-of-the-art quantitative analyses for individual compounds in a complex matrix. It also stresses the need for an SRM such as the shale oil which laboratories can use to guage the accuracy of their analytical methods.

Table 11. Interlaboratory comparison of results of shale oil analyses (ppm).

Compound

pyrene

fluoranthene

phenol

o-cresol

2,4,6-trimethylpyridine

$\begin{array}{rrrrrrr}\text { NBS }^{\mathrm{a}} & \underline{2} & \underline{3} & \underline{4} & \underline{5} & \underline{6} & \underline{7} \\ 107 & 155 & 360 & 150 & 168 & 212 & 141 \\ 61 & 102 & 220 & 80 & 108 & 104 & 112 \\ 395 & 392 & 180 & - & - & - & 399 \\ 338 & 350 & 150 & - & - & - & 381 \\ 1060 & 468 & 460 & - & 950 & - & 1092\end{array}$

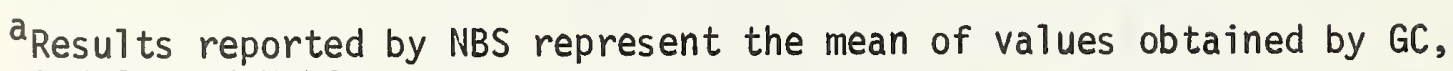
GC/MS, and HPLC. 
2. GC/MS Analysis of Aqueous Effluents from Coal Conversion Processes

L. R. Hilpert, B. F. Howel, K. L. Richie

Analys is of aqueous effluents from coal conversion processes has been initiated with the receipt of our first effluent samples. Two aqueous effluents were received from the Pittsburgh \& Midway Coal Mining Co. Solvent Refined Coal (SRC) Pilot Plant: a BioUnit effluent and a Plant effluent. The Biounit effluent is filtered through sand and charcoal, and then mixed with solidification belt cooling water to generate the Plant effluent.

A simple extraction procedure was used to isolate neutral, acidic, and basic fractions from the samples. Neutral fractions were obtained by extracting $50 \mathrm{~mL}$ of the aqueous effluent with $25 \mathrm{~mL} \mathrm{CH}_{2} \mathrm{Cl}_{2}$. Two additional $50 \mathrm{~mL}$ aliquots of the effluents were adjusted to $\mathrm{pH} 1$ with $\mathrm{H}_{2} \mathrm{SO}_{4}$ and to $\mathrm{pH} 13$ with concentrated $\mathrm{KOH}$, respectively and extracted with $25 \mathrm{~mL}$ of $\mathrm{CH}_{2} \mathrm{Cl}_{2}$ to generate the acidic and basic fractions.

The above fractions (for both the BioUnit and Plant effluents) were analyzed by GC/MS on a $30 \mathrm{HSP}-1000$ high resolution capillary columin. Total ion chromatograms for the acidic, basic, and neutral fractions from the Biounit effluent are shown in Figures 2,3 and 4 . Tentative peak identifications were based on a comparison of the mass spectra gererated during the GC/lis analys is with a library of mass spectra. The chromatograms for the neutral and basic extracts show the same compounds (a series of $n$-al kanes $\sim C_{13}-C_{20}$, substituted pyridines and tetrahydroquiriolines) in the same relative amounts. The major peaks in the acidic fraction are a series of $n$-alkanes $\left(\sim C_{13}-C_{20}\right)$ along with minor amounts of phenols.

Since industrial effluents are routinely monitored for phenols, and since these values reflect to some extent the efficiency of the particular control technology or clean-up method utilized, we decided to determine the concentrations of several phenols in the effluents. Two methods were used to measure phenol and o-cresol concentrations in the Biounit and Plant effluents. The first method involved GC/MIS SIM analys is of the $\mathrm{CH}_{2} \mathrm{Cl}_{2}$ extract using an internal standard. The second method was the ASTM colorimetric procedure.

p-Cresol was chosen as the internal standard in the GC/MS quantitation since preliminary SIM GC/MS analysis of the BioUnit and Flant effluent extracts showed the p-cresol to be present at a level at least lonx lower than either the phenol or o-cresol. The p-cresol was added to aliquots of the Biounit and Plant effluents at the $1 \mathrm{ppm}$ level. The samples were extracted and the acid fractions analayzed by GC/lis with

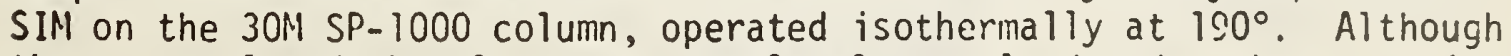
the 0 -cresol and phenol are not completely resolved under these conditions, the use of selected ion monitoring allows determination of each compound free of interference from the other (see figure ${ }^{5}$ ). Phenol and 


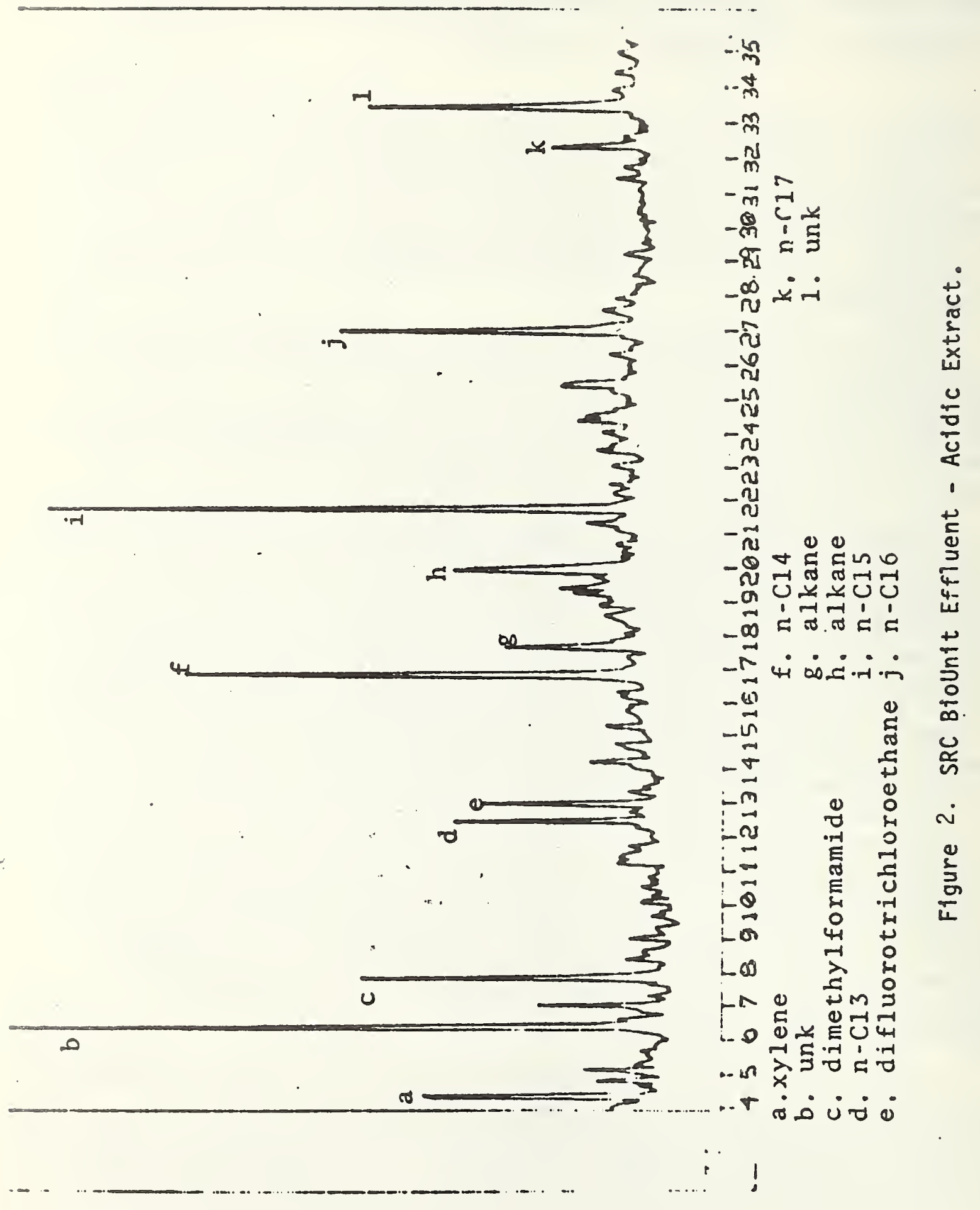




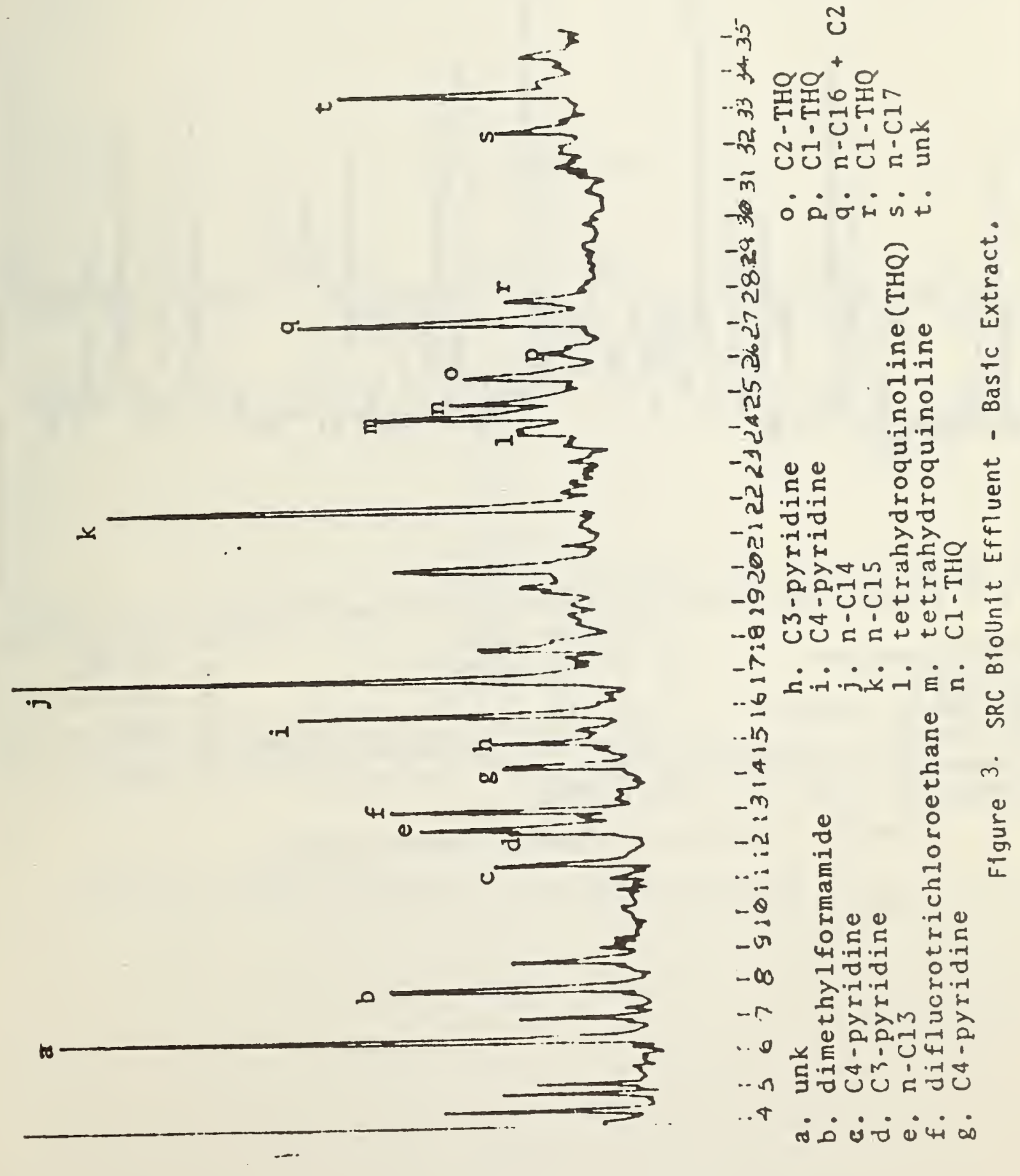




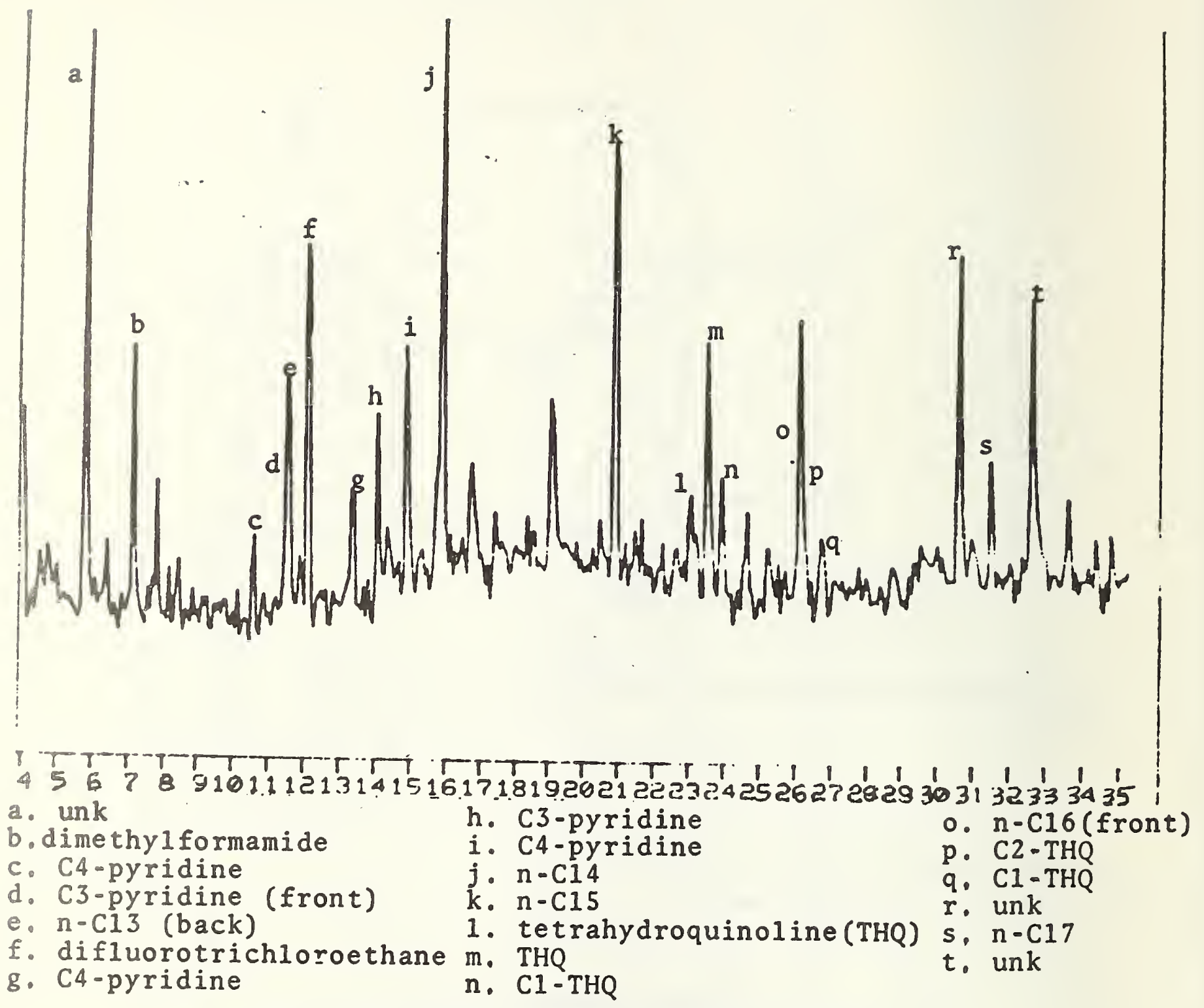

Figure 4. SRC BioUnit Effluent - Neutral Extract 
o-cresol concentrations were determined from the respective peak areas, the internal standard concentration, the extraction recoveries, and the relative response factors for the phenol and 0 -cresol relative to $\mathrm{p}$-cresol which were determined independently. The results of these determinations are summarized in Table 12 .

Table 12. GC/MS analysis of aqueous effluents.

\begin{tabular}{lcc} 
SRC BioUnit Effluent $\mathrm{H}_{2} \mathrm{O}:$ & $\frac{\text { phenol }(\mathrm{ppb})}{38.2 \pm 1.9}$ & $\frac{\text { o-cresol }(\mathrm{ppb})}{3.26 \pm 0.24}$ \\
\hline SRC Plant Effluent $\mathrm{H}_{2} \mathrm{O}:$ & $18.8 \pm 0.6$ & $2.12 \pm 0.2$
\end{tabular}

Total phenol concentrations in the effluents were also determined using ASTM D procedure \#1783-70. Standard aqueous phenol solutions from 10-100 ppb were used to construct a calibration curve. The Biounit and Plant effluent samples were analyzed and the phenol (total) concentrations determined from the calibration curve. The results are summarized in Table 13.

Table 13. Colorimetric analys is of aqueous effluents ASTM D procedure 1783-70.

$\underline{\text { Sample }}$

Biounit effluent

Plant effluent
Phenols conc.

$125 \mathrm{ppb} \pm 20 \%$

$69 \mathrm{ppb} \pm 20 \%$

Whereas the GC/MS quantitation was specific for phenol and 0-cresol, the values obtained using the ASTM procedure represent the totaT phenols in the samples. This may account for the discrepency between the two sets of values, although factors such as potential interferences in the colorimetric assay and non-linearity of the calibration curve must also be considered. 
3. Application of Multi-Dimensional Chromatography to Trace Organic Compound Quantitation

S. N. Chesler, F. R. Guenther

A multi-dimensional gas chromatographic technique has been devised for the analysis of single compounds in complex matrices. A simple switching device was constructed which allows the transferring of a portion of the effluent from one capillary column to another capillary column. Under proper conditions, this transfer can be done without the use of cryogenic trapping on the second column.

This technique was used to certify 5,6-benzoquinoline in the Shale $0_{i 1}$ SRM. An acid/base extraction scheme was used to isolate the $\mathrm{N}$-heterocyclic containing fractions. The fraction was then concentrated under a stream of dry nitrogen to approximately $100 \mu \mathrm{L}$. Two $\mu \mathrm{L}$ was then injected onto the first column, a non-polar SE52 capillary column, to give a boiling point separation. Subsequently, a portion of the effluent containing the compound of interest was transferred from the first column to a carbowax 20M capillary column. Separation on this column is based on polarity. The analyte was easily separated from all other compounds. A standard addition technique was used with phenenthridine as an internal volume corrector. This procedure was used on eight separate groups of ampoules from the SRM lot. The final concentration was determined to be $15.1 \pm 1.2 \mathrm{ppm}$ (one standard deviation). This compares favorably with the liquid chromatographic determination of $16.3 \pm 1.8 \mathrm{ppm}$.

\section{Combined LC/MS Technique for Direct Quantitative Analysis of Individual Organic Compounds in Complex Mixtures}

R. G. Christensen, H. S. Hertz, S. Meiselman, E. White V

An interface for a conventional liquid chromatograph and a conventional mass spectrometer has been constructed which operates on the principle of pre-concentrating a liquid stream and then admitting part of it to the ionization region of the mass spectrometer. The selectivity of the mass spectrometer and the reproducible behavior of the entire system allow the direct quantitation of substances in complex mixtures.

The pre-concentration is accomplished by allowing the effluent from the liquid chromatograph to flow down a heater wire. The evaporation of solvent has yielded up to a 20-fold enrichment of analyte, and it is believed that 100-fold enrichment is feasible.

The flow into the mass spectrometer is controlled by a small needle valve built into the tip of the inlet tube. The valve is formed by a $0.05 \mathrm{~mm}$ teflon film at the tip of the tube pierced by a pointed 0.1 $\mathrm{mm}$ wire which passes throught the bore of the tube. Flow rate into the mass spectrometer can be controlled in the range of $0-20 \mu \mathrm{L} /$ minute. Since all of the pressure drop occurs at the high vacuum end of the tube, boiling within the tube with consequent unstable flow is avoided. 
The stability of the system is such that quantitation can be done with external as well as internal standards. Figure 6 shows typical chromatograms from the determination of phenol in a shale oil matrix using external standards. The level found was $400 \pm 40 \mathrm{ppm}$, a value in good agreement with, and with precision comparable to, two other methods requiring lengthy sample pretreatment. The direct shale oil determination by LC/MS required about 15 minutes per run.

\section{Work Plan and Evaluation/Feasibility Study of Drilling Fluid Reference Standards for Chemical Analysis}

J. R. Moody

\section{Background}

Man's activities in off-shore drilling (as well as on-shore drilling) must proceed in order to locate new sources of oil and gas to satisfy the increasing demands for energy resources. If we are to preserve our renewable resources as we extract oil and gas from the continental shelf, research, development, and monitoring are needed to provide a better foundation for rational decisions on minimizing risks and protecting other resources.

Drilling fluids are now recognized as one of the major factors involved in the success or failure of drilling operations. The composition of a drilling fluid depends upon the formation to be drilled. Drilling fluids normally consist of colloidal suspensions of clay and minerals in water with chemical additives, and are used in production operations to support the borehole, to remove cuttings, and to cool the drill bits. In order to control the physical and chemical properties of drilling fluids, several types of inorganic and organic chemicals are used in conjunction with the clays, minerals, thinning and dispersing agents, etc. Since disposal of the drilling fluids is into water or onto adjacent land, it is essential to characterize these fluids for their chemical constituents in order to evaluate and assess the fate of the chemical constituents (many of them toxic) on the aquatic and terrestrial environment.

Scope

NBS will develop and provide EPA/Gulf Breeze with drilling fluid reference standards for use in characterization efforts and in a quality assurance program to improve and maintain reliability of measurements made on discharges from drilling and production operations.

\section{Mi 1estones:}

(1) Obtain candidate reference material(s) from EPA/Gulf Breeze and assess the feasibility for preparing a dry mixture of four major drilling fluid components

(2) If milestone (1) proves feasible, prepare a batch (i.e., grind, blend, sieve) of material and establish homogeneity 


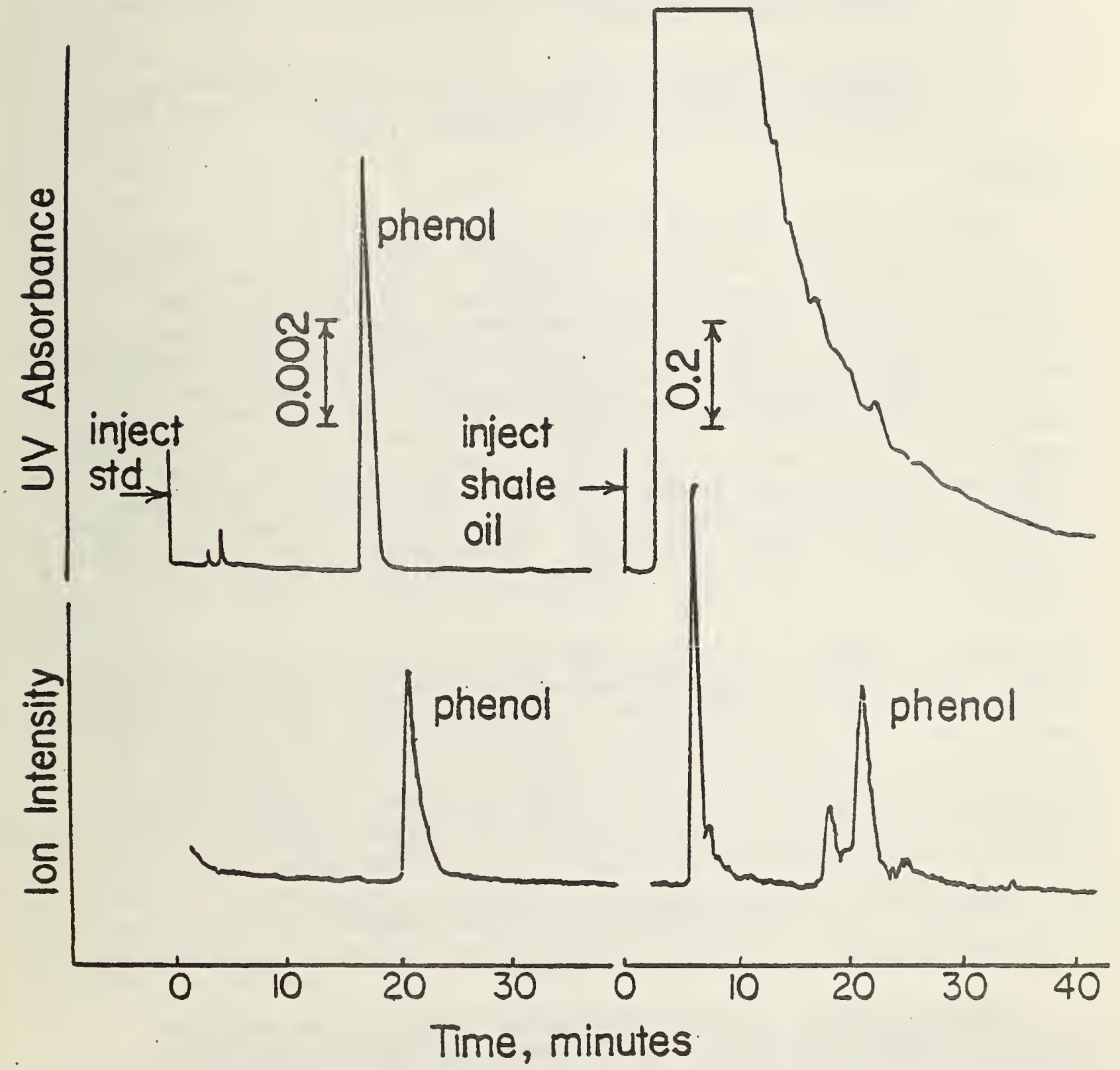

Figure 6. Chromatogram of shale oil obtained during phenol quantitation. Upper trace: ultraviolet detector recording at $254 \mathrm{~mm}$. Lower trace: single ion record at $\mathrm{m} / 294$. 
(3) Characterize the drilling fluid mixture for several trace elements to be determined by NBS and EPA/Gulf Breeze

(4) Package, label, and provide EPA/Gulf Breeze with drilling fluid reference standard for specified trace elements

(5) Initiate studies on a drilling fluid material chosen in concert with EPA/Gulf Breeze for future (FY81) issuance as a more complex or more characterized standard

\section{Progress}

EPA/Gulf Breeze provided NBS with three test "fluids" for feasibility and assessment studies. As a result of these studies, EPA/Gulf Breeze and NBS have agreed that the reference drilling fluid sample should consist of a dry mix of the components found in drilling mud. NBS has ordered and received the five major components of drilling mud barite, bentonite, chrome lignosulfonate, lignin, and caustic soda. The first three components have been blended in a dual $\mathrm{V}$ blender. Samples of this blend will be analyzed to confirm or deny the apparent homogeneity of the sample. Conceivably, some re-segregation of the sample may occur on storage due to the large difference in component densities. Therefore, some effort will be made to determine the degree to which this may happen. Packaging in small units and requiring use of the entire sample in an experiment would be another way around this problem.

If lignin is added in the solid state with caustic soda, two problems arise. First, the oil companies believe that the total mixture of dry components will not mix with water to yield the normal mud. Secondly, caustic soda is hygroscopic and in a flake form (a11 other components are powders, 200-400 mash). It would have to be ground (difficult to do) in order to blend well with the other components. At this point, it would be appropriate to solicit reaction to a three component dry mix because of the problems associated with a 5 component mix.

\section{Atomic Absorption and Flame Emission Spectrometry}

\section{J.D. Messman, T.C. Rains, M.S. Epstein}

The major effort in atomic absorption (AAS) and flame emission (FES) spectrometry has been directed toward the characterization of Standard Reference Materials and the development of reference methods. Research activities have been in the development of continuum source AAS and metal speciation using high performance liquid chromatography-AAS.

As part of the continuing effort to improve NBS analytical expertise in atomic absorption spectrometry, a practical atomic absorption spectrometric system has been developed which utilizes a single continuum source in conjunction with a high resolution, wavelength-modulated echelle spectrometer. This technique can be described as ContinuumSource Echelle Wavelength Modulation-Atomic Absorption Spectrometry (CEWM-AAS). It has been successfully applied to the analysis of NBS Standard Reference Materials as well as other diverse complex samples. 
There are numerous advantages of CEWM-AAS over existing state-ofthe-art commercial atomic absorption instrumentation. Wavelength modulation provides true double beam operation using a simple single beam optical arrangement. Electronic ratioing of the $A C$ component (propotional to $I_{0}-I$ ) of the photosignal to the $D C$ component (proportional to $I_{0}$ ) of the photosignal results in an $A A$ instrument which gives a real time absorbance readout, continuously corrected for source attenuation resulting from background absorption. Systematic error in CEWM-AAS is minimized because of accurate compensation for all known major types of spectral interferences encountered in atomic absorption analyses. Moreover, high levels of nonspecific background interference can be corrected for in CEWM-AAS. This advantage makes direct analyses of low levels of trace metals in complex matrices such as biological fluids and lubricating oils feasible.

The CEWM-AAS allows the utilization of either flame or electrothermal atomization reservoirs while the use of a single continuum source as the external primary source of radiation obviates the expensive library of hollow cathode lamps and electrodeless discharge lamps required of all commercial atomic absorption instrumentation.

Once CEWM-AAS has been critically evaluated in the single element mode, the potential of the CEWM-AAS system for multielement analyses will be thoroughly investigated. Instrumental change from the singleelement mode to the multielement mode of operation is simple and convenient since the echelle spectrometer is easily converted into a 20 channel, direct reading polychromator by merely replacing the single exit slit cassette with a multichannel exit slit cassette. Each of the 20 photomultiplier tubes will be individually interfaced, via analog-todigital converters ( $A D C^{\prime} s$ ), to a PDP 11/34 minicomputer for data acquisition, storage, and reduction.

One obvious benefit of simultaneous multielement AAS is a much greater throughput of routine samples. This allows the analyst to devote more time to improving accuracy and precision of AAS through methods development and studies aimed towards the elimination of potential interferences. Moreover, simultaneous multielement AAS is quite amenable to those applications where several trace metals need to be determined in a limited sample volume such as blood serum from a pediatric patient.

During the past year, CEWM-AAS (operated in the single element mode) has been used to analyze a wide variety of Standard Reference Materials including coal, coal fly ash, estuarine sediments, unalloyed copper, and a synthetic natural water matrix for a number of trace metals. In Table 14, the results from four trace metals ( $\mathrm{Ag}, \mathrm{Cr}, \mathrm{Cu}$, and $\mathrm{Ni}$ ) in a synthetic natural water matrix (SRM 1643a) obtained by CEWMAAS are compared to those values obtained with a commercial line-source AA instrument (Perkin-Elmer Model 603) using electrothermal atomization. When operated in the single element mode, analytical performance of CEWMAAS is shown to be as good as, or better than, that using a commercial single element, line-source atomic absorption instrument. 
Table 14. Trace Elements in Water by Atomic Absorption Spectrometry NBS-SRM 1643a

\begin{tabular}{|c|c|c|}
\hline Element & $\begin{array}{c}\text { Concentration } \\
\mathrm{ng} / \mathrm{mL}\end{array}$ & Standard Deviation ${ }^{a}$ \\
\hline \multirow[t]{2}{*}{$\mathrm{Ag}$} & $2.54^{b}$ & 0.13 \\
\hline & $2.93^{C}$ & 0.19 \\
\hline \multirow[t]{2}{*}{$\mathrm{Cr}$} & $18.5^{b}$ & 1.1 \\
\hline & $17.6^{\mathrm{C}}$ & 0.7 \\
\hline \multirow[t]{2}{*}{$\mathrm{Cu}$} & $18.1^{b}$ & 1.2 \\
\hline & $17.8^{c}$ & 0.7 \\
\hline \multirow[t]{2}{*}{$\mathrm{Ni}$} & $55.9^{b}$ & 4.9 \\
\hline & $56.5^{c}$ & 3.5 \\
\hline
\end{tabular}

\footnotetext{
${ }^{a}$ One standard deviation of six replicate samples

$b_{P E} 603 / H G A-2200 / D_{2}$

${ }^{c}$ CEWM/HGA-2100
}

Activities are underway to develop analytical procedures for the determination of seven trace metals ( $\mathrm{Cd}, \mathrm{Cr}, \mathrm{Cu}, \mathrm{Mn}, \mathrm{Pb}, \mathrm{Ni}$, and $\mathrm{Zn}$ ) in a coal matrix by CEWM-AAS. A subbituminous coal (SRM 1635) typical of the coal matrix found in the western region of the United States, is being used as the control material. Analytical conditions for each metal are being optimized for determination by the CEWM-AAS system operated in the single-element mode using electrothermal atomization. Upon completion of this preliminary study, efforts will focus on the assessment of the accuracy, precision, and linear dynamic range for each metal in SRM 1635 as well as any compromises in the analytical conditions which may be necessary when these seven elements are measured simultaneously by CEWM-AAS.

a. Metal Speciation Using High Performance Liquid Chromatography/Atomic Absorption Spectrometry

J. D. Messman, T. C. Rains

During recent years it has become increasingly apparent that total metal data alone may not provide complete and accurate descriptions of the complicated roles which many metals play in our ecosystem. It is generally believed that alkylated forms of certain metals (e.g., $\mathrm{Hg}, \mathrm{Pb}$, and $\mathrm{Se}$ ) are much more toxic than the inorganic salts of the metals or even the metals themselves. Some microorganisms are capable of chemically 
and biochemically transforming certain toxic elements into more toxic organometallic compounds which may then be released into the environment. Additionally, chemical speciation of trace metals can be a real problem in defining nutrient composition of foods because the biological activity of a metal such as chromium or iron depends on the chemical species to which the metal is bound.

The rapidly increasing interest in metal speciation in diverse scientific disciplines has prompted the immediate need for development of analytical instrumentation which allows the determination of not only the total metal content but also the different chemical forms in which the metal may be distributed. Atomic spectrometric techniques (absorption, emission, and fluorescence) have long been regarded as being sensitive and metal-specific spectrochemical technqiues for total metal analyses are limited in their ability to differentiate between various chemical forms of the metal within the sample. Chromatographic techniques can be successfully employed to separate various chemical forms of the analyte, but poor detector specificity frequently limits their analytical utility when highly complex mixtures are encountered. However, interfacing chromatographic and atomic spectroscopic instrumentation into a single analytical hybrid system which has the combined advantages of both techniques presents a viable means of potentially solving analytical problems which otherwise would not be possible by either individual technique.

In an effort to gain more knowledge about the metal distribution in complex matrices, an investigation has been undertaken using the combined instrumentation of a flame atomic absorption spectrometer interfaced to sample the mobile phase exiting at the outlet of the analytical column of a high performance liquid chromatograph (HPLC). To demonstrate the analytical feasibility of HPLC/AAS for molecular characterization of metals in complex matrices, tetraalkyl lead (TAL) compounds were determined directly in gasoline. Baseline resolution of the TAL mixture consisting of tetramethyl lead (TML), trimethyl ethyl 1ead (TMEL), dimethyl diethyl 1ead (DMDEL), methyl triethyl lead (MTEL), and tetraethyl 1ead (TEL) was effected by reverse-phase HPLC using a 70 percent acetonitrile $\left(\mathrm{CH}_{3} \mathrm{CN}\right) / 30$ percent $\mathrm{H}_{2} \mathrm{O}$ mobile phase. The eluted tetraalkyl lead species were then sequentially atomized in an air/ acetylene flame. The lead absorbance for each TAL compound was measured by atomic absorption spectrometry using the 283.3. nm lead resonance line. Analyses were conducted free of spectral interferences. Detection limits for lead by this hybrid technique were about $10 \mathrm{ng}$ for each TAL compound. Using our instrumental configuration and analytical conditions, the 5 tetraalkyl lead compounds were separated and detected in less than 10 minutes. A number of different commercial gasolines were analyzed for their tetraalkyl lead content. The TAL distribution was found to vary widely among manufacturers.

Atomic absorption spectrometry has been compared in terms of analytical performance to UV absorbance spectrophotometry as a detector for HPLC for the determination of tetraalkyl lead compounds in petroleum refinery products. Preliminary data indicate that for certain applications the metal-specific AA detector is more analytically useful as an 
HPLC detector than UV absorbance spectrophotometry for the study of different metal species in complex matrices. In some cases, the UV absorbance detector is severly plagued by spectral interference at $254 \mathrm{~nm}$ due to coeluting aliphatic, olefinic, and aromatic hydrocarbons of the gasoline matrix which absorb at this wavelength. As shown in figure 7 , a clean chromatograph of tetraalkyl lead compounds in gasoline is obtained with the AA detector because it responds to only lead-containing compounds at $283.3 \mathrm{~nm}$ line.

In order to improve the analytical performance of the HPLC/AAS system described in this preliminary report, it is necessary to optimize the chromatographic conditions and to decrease the dead volume present between the column and the detection system. In addition, the detection limits can be significantly lowered by using a flameless atomic reservoir.

The HPLC/AAS technique can be easily extended to the determination of different organometallic compounds in diverse matrices by simply tuning the monochromator to the analytical line of the metal of interest. This inherent specificity of AAS cannot be realized by existing commercial HPLC spectroscopic detectors. Moreover, the specificity of AAS considerably relaxes the constraints of the analytical conditions required for the separation. With AAS, only the compounds containing the metal of interest need to be separated from one another; the metal-containing compounds need not necessarily be separated from the sample matrix. 


\section{UV Absorbance Defector}

(254 nm)

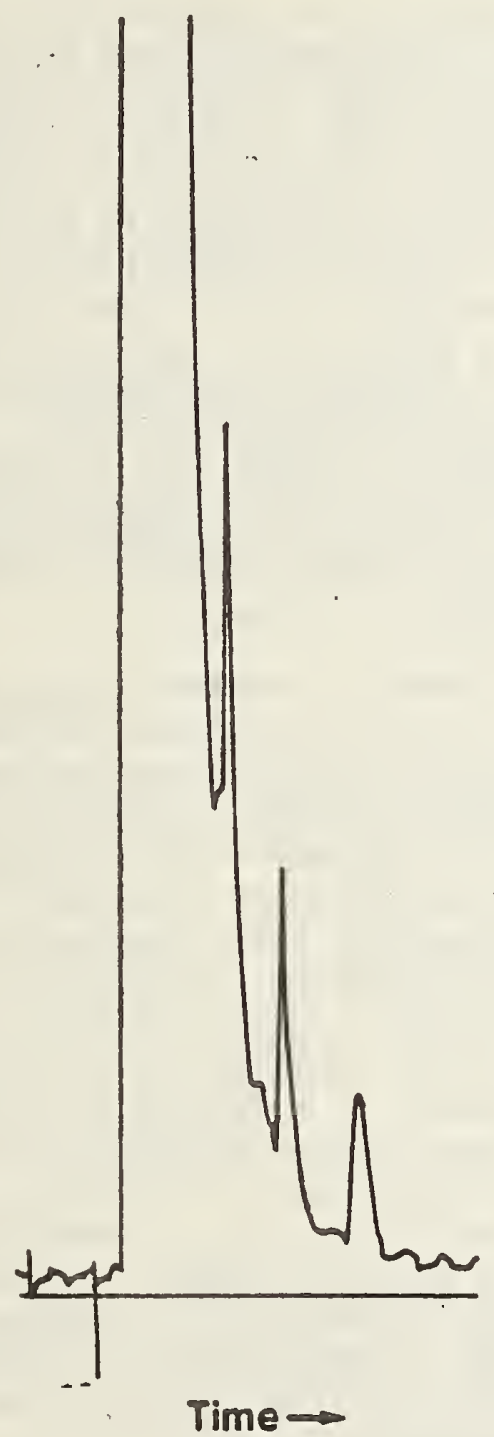

Flame AA Detector

$(283.3 \mathrm{~nm})$

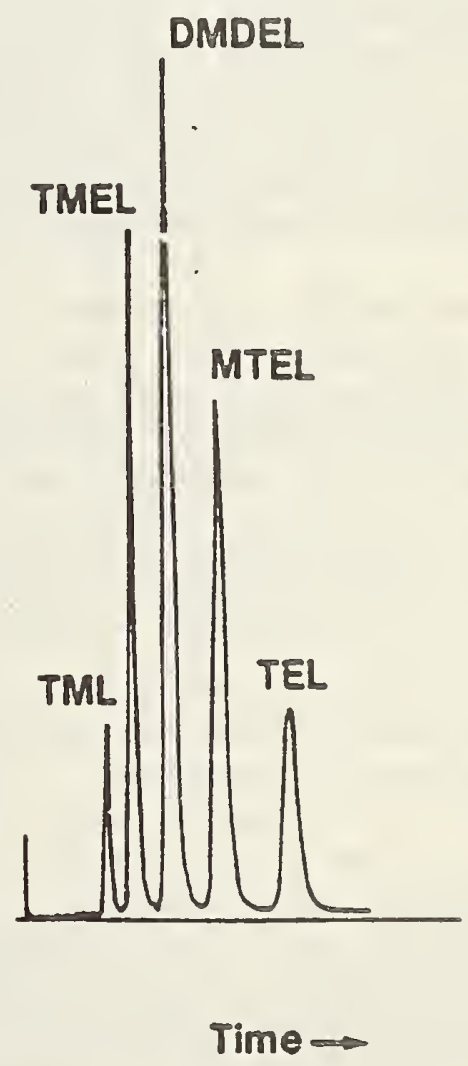

Figure 7. Director Determination of Tetraalkyl Lead Compounds in Gasoline by Reverse-Phase HPLC 
7. Interferences in the Inductively Coupled Plasma (ICP) Technique

R. L. Watters, Jr.

In the application of the ICP technique to high-accuracy analys is of Standard Reference Materials (SRM's), interferences have been found which can degrade the quality of analytical results. These interferences can be traced to spectral and matrix-related interactions which must be characterized and corrected for in order to minimize their effect. The problem of spectral interference in the ICP technique is well known. Procedures for background and spectral overlap corrections have been established and are presently being employed in this laboratory. The first step is to expose a photographic plate with spectra of the sample and pure-element standards. Spectral lines are then chosen from considerations of sensitivity and absence of spectral overlap. The exitslit carriage is mounted on the microdensitometer with the reference photographic plate, and slits are positioned in alignment with the chosen lines on the plate. Final alignment is accomplished by adjustment of a refractor plate in front of each slit.

A wavelength scan of each element is stored in the computer for output on the terminal. From plots of intensity-versus-wavelength for each element, the spectral peak position is checked and background correction points on either side of the peak are chosen. Spectral scanning and background sampling are accomplished by computer-controlled rotation of a refractor plate positioned behind the spectrometer entrance slit. In this manner, true net intensities for the spectral lines are calculated and either stored in the computer for calibration or displayed on the terminal.

Evidence has been found indicating differences in analytical response between pure-element standards and samples of complex matrices which are not due to spectral interference. These effects become significant when applying the ICP technique to high-accuracy analysis of SRM's. Chemical matrix effects in the plasma itself can cause up to fifteen percent relative enhancement or depression of the analyte element intensity depending on the spectral line and plasma operating conditions. Complete definition of the problem has involved examination of compromise conditions for simultaneous multielement analysis both from the point of view of sensitivity as well as matrix effect suppression.

One way to estimate a suspected matrix effect is to record net intensities for various elements at constant concentrations, with and without an excess $(3000 \mu \mathrm{g} / \mathrm{mL})$ of potassium present. The ratio of intensity with potassium to intensity without potassium can be plotted with respect to an instrument variable such as power, or observation height. The objective is to find operating conditions which result in a ratio close to 1.00 without significantly affecting the signal-tobackground ratio. At present, no serious degradation in signal-tobackground ratio has been caused by tuning the ICP for minimum matrix effect. However, the data include only five elements thus far, therefore the results must be considered to be preliminary. This data, as well as its extension to additional elements and matrices, will provide the information necessary to minimize analytical bias in simultaneous multielement analysis using the ICP technique. 
Participating Staff: Center for Radiation Research - J.M.R.Hutchinson, J.R. Noyce

Background/Objective

The assessment of regional and national effects of radionuclides depends on the comparability of empirical data generated throughout the new energy developing industries as well as the existing nuclear power industry. The need for a program to provide scientifically valid reference points from which future radiological environmental degradation can be measured and upon which rational policy decisions may be made has been recognized.

The primary objective of this project is to aid in the development of optimum radiological monitoring program to provide radiological pollutant quality assurance. Studies at NBS leading to the attainment of this objective include: (1) the improvement of radiological monitoring techniques; (2) the evaluation of presently used environmental radiological surveillance programs; (3) the provision of standards for measurement of radionuclides.

FY-1980 Milestones

- Prepare and deliver SRM for thorium-232 - a naturally occuring radionuclide found in coal and likely to be present in coal plant effluents.

- Prepare and deliver a mixed solution SRM (i.e. $3 \mathrm{H},{ }^{90} \mathrm{Sr},{ }^{134} \mathrm{Cs}$, $226,{ }^{228} \mathrm{Ra}$ ) needed to support measurements required by the Interim Drinking Water Regulations.

FY-1980 Accomplishments

1. 232 Th Solution

Thorium-232 is a radionuclide whose presence is of concern when in effluent and run-off waters from some types of mines, ore mills, and tailing piles, and in certain drinking water supplies. This calibrated solution of $232 \mathrm{Th}$ is for laboratories that assay these waters. The starting material was all thorium nitrate, chosen so that the ${ }^{232}$ Th progeny would be in radioactive equilibrium. The 232 Th content was assayed by isotopedilution mass spectrometry and confirmed by $\gamma$-ray counting. Two impurities were observed and measured: ${ }^{230}$ Th by means of $\alpha$-particle spectrometry with a silicon surface-barrier detector, and ${ }^{226} \mathrm{Ra}$ by means of $\mathrm{Ge}(\mathrm{Li})$ detector spectrometry. The $\alpha$-particle spectrum is shown in Figure 8 , and the Report of Calibration is given at the end of this section. Two hundred ampoules of this solution were provided to EPA-LV.

\section{Mixed Radionuclide Solutions}

Two mixed radionuclide solutions were needed by EPA to distribute to laboratories that assay for radionuclides in drinking water. One solution mixture is a ten-fold dilution of the other. Each mixture contains ${ }^{3} \mathrm{H},{ }^{90} \mathrm{Sr}-{ }^{90} \mathrm{Y},{ }^{3{ }^{4}} \mathrm{Cs},{ }^{226} \mathrm{Ra}$ and $i t s$ progeny, and natural uranium and its progeny. The ${ }^{228} \mathrm{Ra}$ and natural uranium components were developed 
earlier in this multi-year program; the ${ }^{134} \mathrm{Cs}$ solution was calibrated specifically for these mixtures. A Report of Calibration for one of the mixtures is given at the end of this section. 


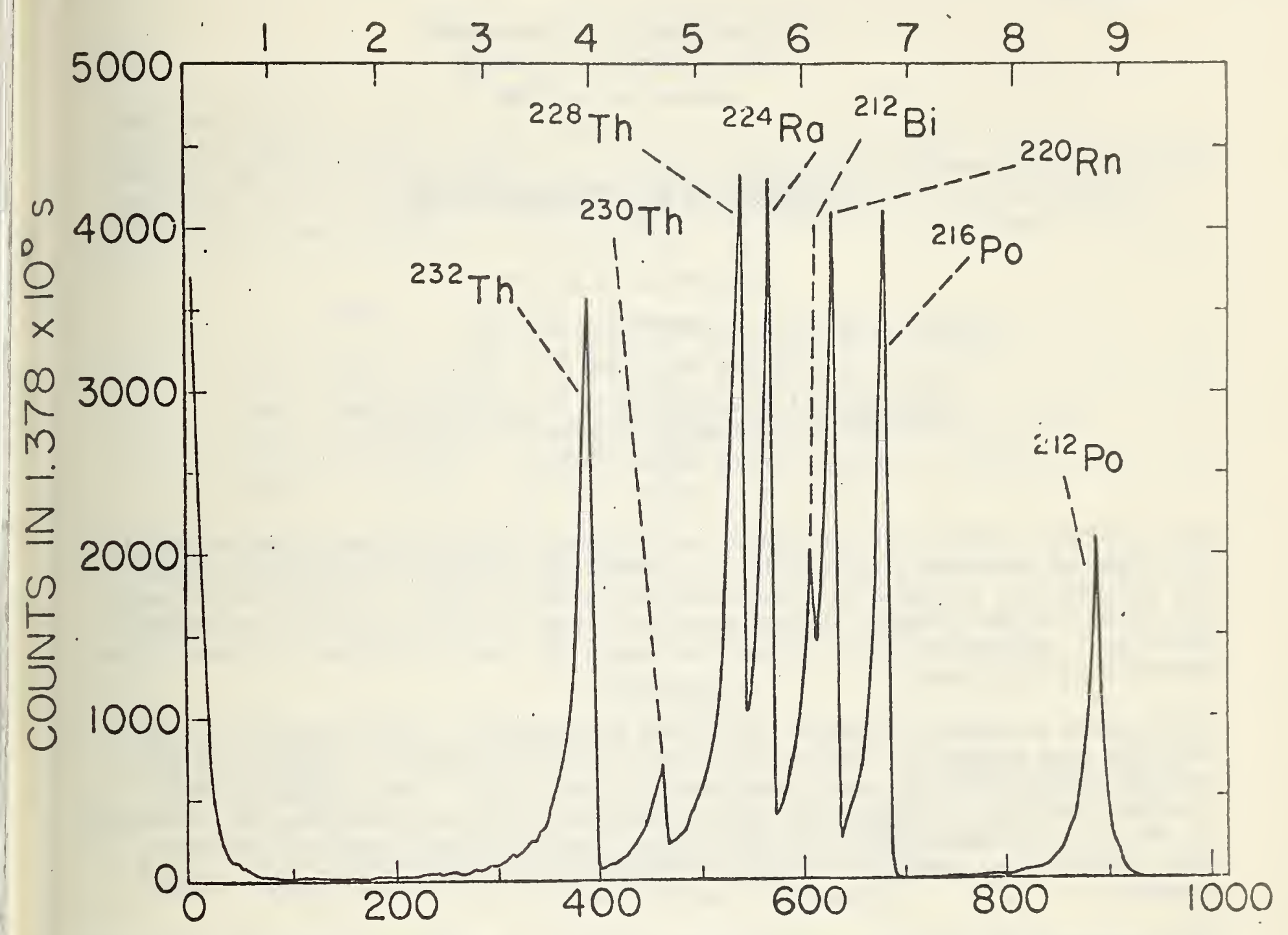

CHANNEL NUMBER

Figure 8. ALPHA PARTICLE ENERGY, MeV 


\author{
U.S. DEPARTMENT OF COMMERCE \\ NATIONAL BUREAU OF STANDARDS \\ WASHINGTON, D.C. 20294
}

\title{
REPORT OF CALIBRATION
}

\author{
THORIUM-232 SOLUTION \\ prepared for the \\ Quality Assurance Division \\ Environmental Monitoring and Support Laboratory \\ U.S. Environmental Protection Agency \\ Las Vegas, Nevada
}

This solution consists of thorium-232 and progeny in equilibrium in approximately 4.0 grams of one-molar nitric acid in a flame-sealed borosilicate-glass ampoule. The solution was prepared by dissolving old thorium nitrate in dilute nitric acid and filtering the solution through a membrane-type filter having 0.3 -micrometer openings. Information from the supplier about the thorium nitrate is given in the appendix to this Report.

Two hundred and twenty ampoules containing approximately equal portions of this solution were prepared. Six of these ampoules were assayed for thorium concentration by isotope-dilution mass spectrometry in May 1979. These assays by the Inorganic Analytical Research Division, National Bureau of Standards, are described in the attached memorandum. The mean value from the assays is $0.04738 \mathrm{gram}$ of total thorium per gram of solution. The total uncertainty in this value is 0.5 percent.

The radioactivity concentration of thorium- 232 in the solution, which was calculated from the mass-spectrometric value and the conversion factors and constants in Table 1 , is

$$
\star 1.92 \times 10^{2} \mathrm{~s}^{-1} \mathrm{~g}^{-1} \pm 1.8 \% * \text {. }
$$

In making this conversion, all of the thorium was assumed to be thorium-232, on a mass basis.

The uncertainty in the radioactivity concentration, 1.9 percent, is the linear sum of the total uncertainty in the mass-spectrometric value and three times the uncertainty in the thorium-232 half life.

The thorium nitrate from which this solution was prepared contains radium-226 and thorium-230 impurities. An ampoule of the solution was assayed for thorium-232 and radium-226 by measuring the emission rates of gamma rays from the decay of their respective progeny with a $\mathrm{Ge}(\mathrm{L} i)$-detector spectrometer. The radioactivity concentraof thorium-232 obtained from this assay agreed, to within four percent, with the value derived from the mass-spectrometric assays. The ratio of the radioactivity concentration of radium-226 to that of thorium-232 was $0.010 \pm 0.003$ in July 1979 . 
The thorium-230 to thorium-232 activity ratio of a dried deposit of this solution was calculated from the results of silicon surface-barrier-spectrometer measurements to be $0.15 \pm 0.01$. The radioactivity concentration of uranium-238 was estimated from the alpha-particle spectra to be less than 0.5 percent of that of thorium-232, and likewise for uranium-234.

\section{TABLE 1. RELEVANT CONVERSION FACTORS AND CONSTANTS}

Half life of $232 \mathrm{Th}=(1.405 \pm 0.006) \times 10^{10} \mathrm{y}$

Atomic mass of $232 \mathrm{Th}=(232.038053805 \pm 0.000002515) \mathrm{amu}$ (2)

Avogadro constant $\quad=(6.0220978 \pm 0.0000010) \times 10^{23}(3)$

Length of tropical year $=3.155693 \times 10^{7} \mathrm{~s}(\mathrm{a})$

(a) Based on the ephemeris second.

\section{REFERENCES}

1) M. R. Schmorak, "Nuclear Data Sheets for $A=232,236,240 "$, Nucl. Data Sheets, 20, 165 (1977).

2) A. H. Wapstra and K. Bos, "The 1977 Atomic Mass Evaluation, Part I. Atomic Mass Table", Atomic and Nucl. Data Tables, 19, 177 (1977).

3) Recent, unpublished value, National Bureau of Standards.

For the Director,

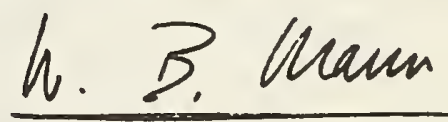

W. B. Mann, Principal Scientist

Radioactivity Group

Center for Radiation Research

March 12, 1980 


\section{APPENDIX TO REPORT OF CALIBRATION \\ THORIUM-232 SOLUTION}

The following set of data about the thorium nitrate used for this solution was provided by the supplier, and is given here for information only.

The thorium in the compound, $\operatorname{Th}\left(\mathrm{NO}_{3}\right)_{4} \cdot 6 \mathrm{H}_{2} \mathrm{O}$, was purified in 1906. Spectrophotometric assays of the compound in 1954 gave these results for some impurity metals.

\begin{tabular}{cc} 
Element & Content, ppm \\
\cline { 1 - 2 } Li & $<5$ \\
$\mathrm{Na}$ & $<25$ \\
$\mathrm{~K}$ & $<5$ \\
$\mathrm{Ca}$ & 3 \\
$\mathrm{Rb}$ & $<5$ \\
$\mathrm{Sr}$ & $<3$ \\
$\mathrm{Cs}$ & $<25$ \\
$\mathrm{Ba}$ & 3
\end{tabular}




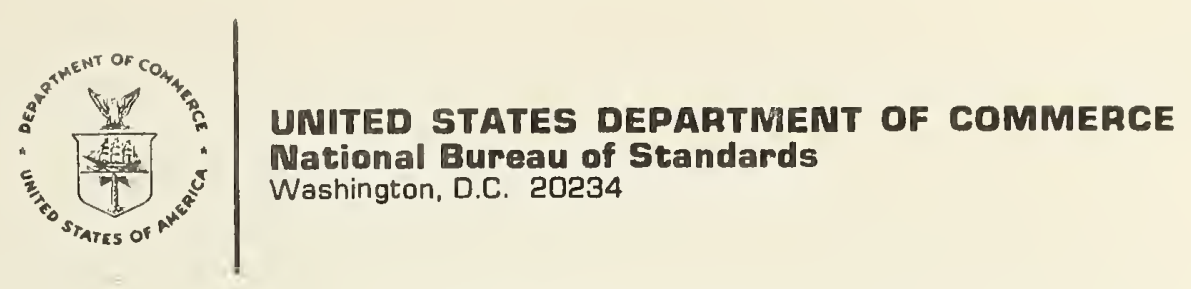

June 11,1979

MEMORANDUM FOR Jim Noyce

Through: E. L. Garner

From: J. W. Gram1ich and L. A. Machlan lywg

Subject: Mass Spectrometric Analysis of Thorium Samples Submitted by Jim Noyce

The concentration of thorium has been determined in six ampoules of thorium nitrate solution by isotope dilution mass spectrometry. Approximately $1 \mathrm{~g}$ of solution was withdrawn from each of the six ampoules and transferred by weight to $200 \mathrm{~mL}$ flasks. These solutions were diluted by weight to approximately $180 \mathrm{~g}$ with $0.5 \mathrm{~N} \mathrm{HNO}_{3}$. After thoroughly mixing, approximately $1 \mathrm{~g}$ of aliquotes were withdrawn and transferred by weight to Teflon beakers. A second aliquot was withdrawn from two of the flasks for duplicate assay and a second aliquot was withdrawn from two other flasks for determination of the isotopic composition. A weighed aliquote of a $230 \mathrm{Th}$ solution of known concentration was added to each of the eight samples for isotope dilution analysis, and after mixing the solutions were evaporated to dryness.

The samples were analyzed by thermal ionization mass spectrometry using a triple filament rhenium source with a zone refined rhenium ionizing filament. Three blanks processed with the samples averaged less than $0.2 \mathrm{ng}$ of thorium. The two isotopic composition samples yielded a $23^{32} \mathrm{Th} /{ }^{230} \mathrm{Th}$ ratio of greater than $1 \times 10^{5}$. The results of the isotope dilution analysis is reported in the accompanying table. 
Concentration of Thorium in Thorium Nitrate Solutions

Sample No.

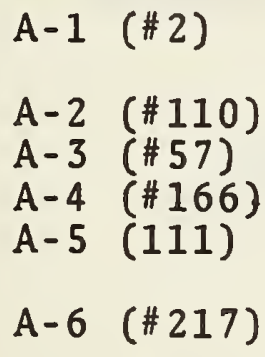

Aliquot

1

2

1

1

1

1

2

1
Percent Th

4.738

4.745

4.730

4.746

4.736

4.742

4.740

4.728

Average $=4.738$

$s=0.0066$

The total uncertainty in the average percent thorium by weight, 0.5 percent, is the linear sum of $0.2_{5}$ percent, which is the uncertainty (at the 95-percent confidence limits) in the concentration of the thorium-230 spike, and $0.2_{5}$ percent, which is the uncertainty (at the 95-percent confidence limits) in the mass-spectrometric measurements for thorium-232. 


\title{
U.S. DEPARTMENT OF COMMERCE
}

NATIONAL BUREAU OF STANDARDS

WASHINGTON, D.C. 20224

\section{REPORT OF CALIBRATION}

\author{
MIXED 'RADIONUCLIDE CALIBRATED SOLUTION M2 \\ prepared for the \\ QUALITY ASSURANCE DIVISION \\ U.S. ENVIRONMENTAL PROTECTION AGENCY \\ LAS VEGAS, NEVADA
}

This calibrated solution consists of hydrogen- 3 , strontium-90-yttrium-90, cesium134, radium-226 and its progeny, radium-228 and its progeny, and uranium of natural isotopic composition and its progenies in approximately $4.2 \mathrm{~g}$ of carrier solution in a flame-sealed, borosilicate-glass ampoule. The carrier solution is $1 \mathrm{M} \mathrm{HNO}_{3}$ containing approximately $10 \mu \mathrm{g}$ each of stable strontium, yttrium, cesium, and barium per gram of solution, and is approximately $0.1 \mathrm{M}$ in $\mathrm{HCl}$. The ampoule contains part of a master solution which was prepared by the gravimetric addition of a quantitative mixture of individually calibrated solutions of the radionuclides to a known mass of the carrier solution.

The radioactivity concentrations on January 1, 1980, the total uranium concentration by mass, their uncertainties, and the half lives used in the decay calculations are given in the attached table.

The hydrogen-3 used in the preparation of this mixture was Standard Reference Material (SRM) 4927-B. The strontium-90-yttrium-90 was a portion of the solution from which SRM 4919-D was quantitatively prepared. The activity of the cesium-134 solution was measured using the National Bureau of Standards (NBS) pressurized " $4 \pi$ " $\gamma$ ionization chamber that had previously been calibrated with a cesium-134 standard in terms of a radium-226 reference source. The radium-226 used in the preparation of this mixture was a portion of the solution from which SRM 4953-B was quantitatively prepared. The radium-228 was the NBS calibrated solution of July 1977. The uranium in the mixture came from the NBS calibrated solution of natural uranium of November 1978, plus part of the more concentrated solution from which the 1978 uranium solution was quantitatively prepared.

The total uncertainty in the radioactivity concentration of each radionuclide and in the total uranium concentration by mass is the linear sum of the 99-percent confidence limits for the mean and the estimated upper limits of conceivable systematic errors in the calibration, the decay calculation (where applicable), and the preparation of this solution. 
Each radioactive component of the mixture was examined for gamma-ray-emitting impurities using germanium-spectrometer systems that covered the energy region from 90 to $1900 \mathrm{keV}$. Radium-226 and its progeny were observed in the radium-228 and uranium solutions. Radium-228 and its progeny were observed in the uranium solution. The radium-226 and radium-228 impurities are included in the total radioactivity concentrations reported for these nuclides. No other gamm-ray-emitting impurities were identified.

Attachment

March 1980
For the Director,

$$
\text { h. 3. Maun }
$$

W.B. Mannn, Principal Scientist Radioactivity Group Center for Radiation Research 
MIXED RADIONUCLIDE CALIBRATED SOLUTION M2

\begin{tabular}{|c|c|c|c|c|c|}
\hline \multirow{2}{*}{$\begin{array}{l}\text { PARENT } \\
\text { RADIO- } \\
\text { NUCLIDE }\end{array}$} & \multirow{2}{*}{$\begin{array}{l}\mathrm{s}^{-1} \mathrm{~g}^{-1} \text { ON } \\
\text { JAN. } 1,1980\end{array}$} & \multicolumn{3}{|c|}{ UNCERTAINTY, \% } & \multirow[b]{2}{*}{$\begin{array}{l}\text { HALF LIFE, } \\
\text { YEARS }\end{array}$} \\
\hline & & $\begin{array}{l}\text { RANDOM } \\
\text { (99\% C.L.) }\end{array}$ & $\begin{array}{l}\text { SYSTEM- } \\
\text { ATIC }\end{array}$ & TOTAL & \\
\hline $3_{H}$ & 236.1 & 0.2 & 0.6 & 0.8 & $12.35 \pm 0.01(b)$ \\
\hline $90 s r$ & 1.45 & 1.3 & 0.9 & 2.2 & $29.12 \pm 0.24(b)$ \\
\hline${ }^{134} \mathrm{cs}$ & 10.47 & 0.4 & 1.3 & 1.7 & $2.062 \pm 0.005(b)$ \\
\hline${ }^{226} \mathrm{Ra}$ & 0.891 & 0.1 & 1.7 & 1.8 & $1600 \pm 7(b)$ \\
\hline${ }^{228} \mathrm{Ra}$ & 0.731 & 1.4 & 2.0 & 3.4 & $5.75 \pm 0.03(c)$ \\
\hline $234 U$ & 1.22 & 3.9 & 1.4 & 5.3 & $\begin{array}{l}(2.445 q \pm 0.010) \\
\times 105(c)\end{array}$ \\
\hline $235_{U}$ & 0.05872 & 0.1 & 0.4 & 0.5 & $\begin{array}{l}(7.038 \pm 0.005) \\
\times 10^{8}(d)\end{array}$ \\
\hline $238_{U}$ & 1.272 & 0.1 & 0.4 & 0.5 & $\begin{array}{l}(4.468+0.003) \\
\times \quad 109(c)\end{array}$ \\
\hline TOTAL U & $0.1031(a)$ & 0.1 & 0.2 & 0.3 & - - \\
\hline
\end{tabular}

(a) Milligrams of $U$ per gram of solution, by isotope-dilution mass spectrometry. The radioactivity concentrations of the uranium isotopes were determined from this concentration, the isotopic abundances measured by mass spectrometry, and the respective uranium half lives.

(b) NCRP Report No. 58, App. A, National Council on Radiation Protection and Measurements, Washington (1978).

(c)D.C. Kocher, ORNL/NUREG/TM-102, Oak Ridge National Laboratory, Oak Ridge, TN (1977).

(d)M.R. Schmorak, Nucl. Data Sheets, 21, 91 (1977). 


\section{a. Overview}

A major emphasis in the recent water pollution legislation is upon protecting human health and natural resources by controlling toxic and hazardous substances. With our advancing industrial economy and the emerging of new technologies, thousands of substances are being used which did not exist until the last few decades. As a result, to control pollutants in our Nation's waters we must be able to predict their behavior and effects in such an environment. The development of testing procedures is necessary to provide this predictive capability. The activities in this task involve the development of new and improved methodologies and instrumentation needed to identify, characterize, and quantify constituents in aqueous effluents.

\section{Objective}

Develop new and improved measurement methods and associated instrumentation for the measurement of water pollutants and to assess the performance of special field studies and analyses related to characterizing the levels of water pollutants injected into the environment by new technologies and energy developments; and provide verified data on the chemical and physical properties of water pollutants and the relationship of these properties to concentration of pollutants in effluents with concentrations of pollutants in natural bodies of water.

\section{Interaction}

Strong interactions are maintained with other units of the NBS community - Center for Analytical Chemistry, Office of Standard Reference Materials, Office of Standard Reference Data, Center for Radiation Research, Center for Thermodynamics and Molecular Science, Center for Materials Science, Center for Mechanical Engineering and Process Technology, Center for Applied Mathematics and with other Federal agencies - (EPA, DoE, NOAA, DOI). The activities in this task also involve interactions with industry, state and local governments, research laboratories, universities, as well as with other organizations (e.g., Council on Environmental Quality, ASTM) engaged in related activities which impact the development of standards for water pollution measurements.

\section{Task Justification}

The national effort in water pollution abatement involve municipal and state government agencies, industries, water suppliers and nearly every department of the Federal government. In response to water pollution legislation (P.L. 92-500; P.L. 93-523; P.L. 92-516; etc.), there has been an increase in water monitoring. The number of water pollutants requiring surveillance is growing rapidly as a result of new technologies, and energy development effluent limitations call for monitoring of 40 different parameters for 40 industrial categories. Effluent limitations for $65 \mathrm{classes}$ of toxic substances for 21 industrial classes 
are being issued by the Environmental Protection Agency. Measurement techniques are either inadequate or do not exist to characterize and/ or determine the low concentration levels of pollutants in effluents.

b. List of Projects

\section{Project Title}

(1) Methods Development for Organic Analys is

(2) Methods Development for Inorganic Analysis
Source of

Funding

E/E-EPA

E/E-EPA

and

EPA Chesapeake Bay 
(1) Methods Development for Organic Analysis

Participating Staff: Center for Analytical Chemistry and Center for Thermodynamics and Molecular Science

Background/Objectives

In order to evaluate the environmental impact of expanded or new technologies, measurement techniques and monitoring systems are required in the development of a compatible data base to evaluate the total effects of energy development upon our environment and to make rational policy decisions. Measurement techniques are either inadequate or do not exist to characterize and/or determine the low concentration levels of organic pollutants. Advanced measurement methods and instrumentation need to be developed to enable accurate measurement of energy related organic pollutants, as well as, data quality assurance of special field studies and analyses related to levels of pollutants injected into the environment.

The activities in this project focus on the development of measurement methods and instrumentation to enable the analys is of trace organic pollutants. Research efforts also focus on developing and applying Liquid Chromatography - Electrochemical Detection (LC-EC) to the characterization of effluents for trace organometals. Research efforts in this project compliment research at EPA to achieve a means of sampling and analyzing pollutants in water from energy related technologies.

FY-1980 Milestones

- Support the ERL/Athens laboratory in its major project-development of a master analytical scheme for the analysis of all gas chromatographicalle organic compounds in waters associated with effluents from energy-related industries.

- Continue method development/refinement using LC-MS and GC-MS technology to enable analysis of trace volatile and non-volatile organic pollutants in water.

- Development of advanced measurement and/or field monitoring techniques for non-volatile organic pollutants in effluents.

\section{Selected Technical Highlights}

1. Synthesis, Isotopic and Purity Analyses, and Preparation of Solutions of Stable Isotope Labeled Marker Compounds for Priority Pollutant Analysis

S.N. Chesler, B. Coxon, P. Konash, W. E. May, R. M. Parris, L. T. Sniegoski, M. J. Welch, E. White V

A "Master Analytical Scheme" being developed for the Environmental Protection Agency by the Research Triangle Institute and Gulf South Research Institute is intended to allow qualitative and quantitative analys is of organic chemicals in water by gas chromatography/mass spectrometry. The "Master Analytical Scheme" requires the use of 
internal standards to be developed by NBS. The standards each consist of seven to eleven deuterated compounds in a solvent. Three different mixtures for "purgeable," extractable," and "intractable" organic compounds, each at two concentration levels (ranging from $0.25 \mathrm{\mu g} / \mathrm{L}$ to $2500 \mu \mathrm{g} / \mathrm{L}$ per component), are needed.

of the 25 deuterated compounds required, two 2-aphthalenesulfonic acid- $d_{7}$ and dodecylphosphate $-d_{25}$, were synthesized at NBS because they were not commercially available. The reaction sequences used are as follows:

2-Naphthalenesulfonic Acid-d

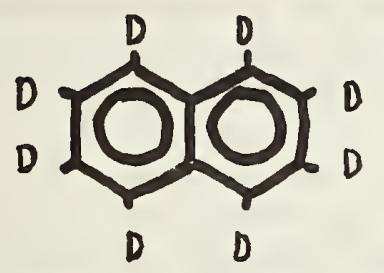

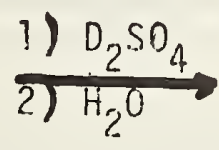

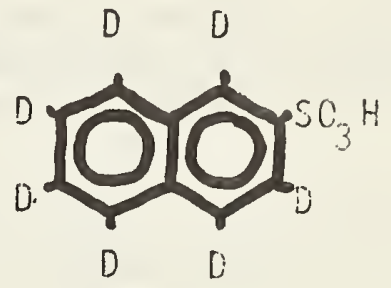

Dodecyl Phosphate- $\mathrm{d}_{25}$

$$
\begin{aligned}
& \mathrm{CD}_{3}\left(\mathrm{CD}_{2}\right)_{17} \mathrm{OD} \frac{\left(\mathrm{C}_{6} \mathrm{H}_{5} \mathrm{O}\right)_{2} \mathrm{POCl}}{\text { pyridine }} \mathrm{CO}_{3}\left(\mathrm{CD}_{2}\right)_{17} \stackrel{0}{\mathrm{H}} \mathrm{OP}\left(\mathrm{OC}_{6} \mathrm{H}_{5}\right)_{2} \\
& \frac{\mathrm{H}_{2} / \mathrm{PC}}{\mathrm{HOAC}} \rightarrow \mathrm{CD}_{3}\left(\mathrm{CD}_{2}\right)_{17} \stackrel{\stackrel{\mathrm{O}}{\mathrm{O}}(\mathrm{OH})_{2}}{2}
\end{aligned}
$$

All dehydrated compounds were checked for chemical purity by mass spectrometry and either gas or liquid chromatography, and for isotopic purity by mass spectrometry.

Since some of the compounds were potentially unstable when stored in mixtures, several test mixtures similar to the internal standards, but of undeuterated compounds in deuterated solvents, were prepared. These were subjected to conditions to accelerate aging, and examined by nuclear magnetic resonance spectrometry. Although some decomposition was observed, the rates were such that properly stored mixtures are expected to be usable for the required several months of evaluation of the "Master Analytical Scheme."

Approximately 600 individual sealed vials of the various internal standard mixtures were prepared, and the 400 required for initial tests of the "Master Analytical Scheme" have been delivered. The remaining vials have been retained at NBS for stability tests, which are in progress. 
2. Fluoroimmunoassay Procedure Developed for Dinitrophenols in Water

\section{P. Cooper, D. J. Reeder, R. Schaffer}

Detection of trace amounts of the toxic pollutant 2,4-dinitrophenol in water is an analytical problem that presents many challenges to the analytical chemist. Laboratory investigations have shown that gas chromatography and high-performance liquid chromatography, while suited for analytical determinations of most of the toxic pollutants, are not as efficient in the separation and identification of the nitrophenols. The development of an immunochemical procedure for detection and quantitation of dinitrophenols in water has been the focus of efforts during 1980.

Development of suitable reagents for the assay entailed the preparation of a suitable material (immunogen) to be injected into rabbits for production of antibodies. Highly purified bovine albumin was combined with dinitrophenol and characterized. The immunogen most successful in eliciting antibodies had 23 dinitrophenyl groups per mole of albumin. The antiserum was produced under contract by a commercial supplier who injected rabbits with the prepared immunogen and bled them periodically for determining antibody development. A procedure that used double diffusion of antiserum versus the immunogen in a gel matrix was the preferred method for following antibody development.

Specific immunoglobulins ( $I g G$ ) were separated from serum by use of an ion-exchange gel preparation. Purity of the IgG was ascertained by use of cellulose acetate strip electrophoresis. The IgG was then covalently bound to solid-phase polymeric beads by coupling with a carbondiimide reagent.

The antibody, thus attached to the beads, was able to bind 2,4dinitrophenol. However, in order to use this capability in a quantitative assay, a compound that could act as a tracer needed to be synthesized. In order to be suitable, the tracer requires dual functionality, i.e., dinitrophenol reactivity as well as physical properties that can be measured, such as radioactivity or fluorescence. A dinitrophenolated fluorescent tracer with dichlorotriazinylaminofluorescein as the fluorescent moiety is currently being synthesized. Alternatively, the use of other fluorescein derivatives, such as fluorescein isothiocyanate, are being considered as substrates for dinitrophenylation. 
The first tracer that we have had success in using in a quantitative competitive assay is a double-labeled rabbit albumin molecule to which both dinitrophenol groups and fluorescein isothiocyanate chromophores are covalently bound. In the competitive assay, the tracer and free dinitrophenol compete for antibody sites on the specific antibody solidphase beads. Maximum sensitivity of the assay is determined by the amount of antibody-bound beads added, the quantity of the competing tracer in the mixture, and the time and temperature of assay incubation.

The solid-phase beads are separated by centrifugation, washed, and then counted in an instrument designed for photon counting of fluorescence at $510 \mathrm{~nm}$ with excitation at $490 \mathrm{~nm}$. Binding curves are then generated from the resulting counts. Typical specific counts range from 30,000 to 600,000 in a two-second counting period, with background and control counts ranging from 15,000 to 25,000 . Plots of the binding versus the amount of dinitrophenol added are linear over two orders of magnitude. Work is now focused on extending the sensitivity range of the assay.

By combining the specificity of the assay with capabilities of concentrating dinitrophenols with an affinity "accumulator" column, detection of ultra-trace amounts of dinitrophenol pollutants in water should be achieved. This will be of importance in the monitoring of industrial effluents.

3. Correlation of Liquid Chromatographic Retention Data with Molecular Shapes for Polycyclic Aromatic Hydrocarbons

W. J. Bonnett, F. R. Guenther, W. E. May, S. A. Wise

Reverse-phase liquid chromatography (LC) on chemically-bonded $C_{1}$ stationary phases is presently the most popular Liquid chromatographlo separation mode. The mechanism of separation on these $C_{18}$ materials has not been established and is a topic of much discussion and research. 
The study of the reverse-phase retention characteristics of polycyclic aromatic hydrocarbons (PAH's) provides valuable information because (1) PAH's are ideal model compounds for investigating the mechanism of retention due to their nonpolarity, and (2) the separation and identification of PAH's are of environmental interest because of the production of PAH's during combustion of fuel and their suspected carcinogenic properties.

Reverse-phase $L C$ on $C_{18}$ phases provides unique selectivity for the separation of isomeric PAH's and particularly methyl-substituted PAH's. A relationship was observed between the shape of PAH solutes, particularly the length-to-breadth ratios and the reverse-phase LC retention on $C_{18}$ bonded phases. In nearly all cases, this ratio was successful in predicting the LC elution order of isomeric PAH's for more than 40 unsubstituted and 40 methyl-substituted PAH's, i.e., the retention increases with increasing length-to-breadth ratio. These ratios were found to be particularly useful in predicting the selectivity of reverse-phase LC for methyl-substituted PAH's. The linear correlation for the LC retention vs the length-to-breadth ratio for 23 methylsubstituted isomeric benzo[c]phenanthrenes, benz[a]anthracenes, and chrysenes is illustrated in figure 9 (correlation coefficient $=0.936)$.

This relationship suggests the importance of solute shape in the LC mechanism. In GC, a similar relationship exists between shape and retention on liquid crystal phases, suggesting the possibility of a liquid crystal effect or "ordering" of the $C_{18}$ phase in LC. The retention characteristics on polymeric $C_{18}$ phases exhibit greater selectivity and a greater correlation with the shape data than on the monomeric phases. This observation indicates that polymeric phases may be more "ordered" than the monomeric phases.

\section{Liquid Chromatography with Electrochemical Detection (LC-EC)}

\section{W. A. MacCrehan}

Research emphasis in this past year has been focused on the development of new chromatographic separations and the optimization of the electrochemical detector response.

Following a literature survey by $A$. Cohen (NBS/CAC), the electroactivity of over 30 currently used organic pesticides was investigated using current-sampled polarography to provide a basis for their electrochemical detection. A chromatographic separation of six frequently encountered pesticides (including parathion and guthion) has been developed (see figure 10).

Many possible separation conditions have been evaluated for the separation of the cationic organotin species, employing such methods as ion exchange and reverse-phase chromatography in the presence of complexing agents. Little success has been obtained in this laboratory, but Brinckman and Jewett have developed a separation for the monocationic species, and this separation was used to evaluate the electrochemical detection approach. The amperometric detection of triphenyltin 


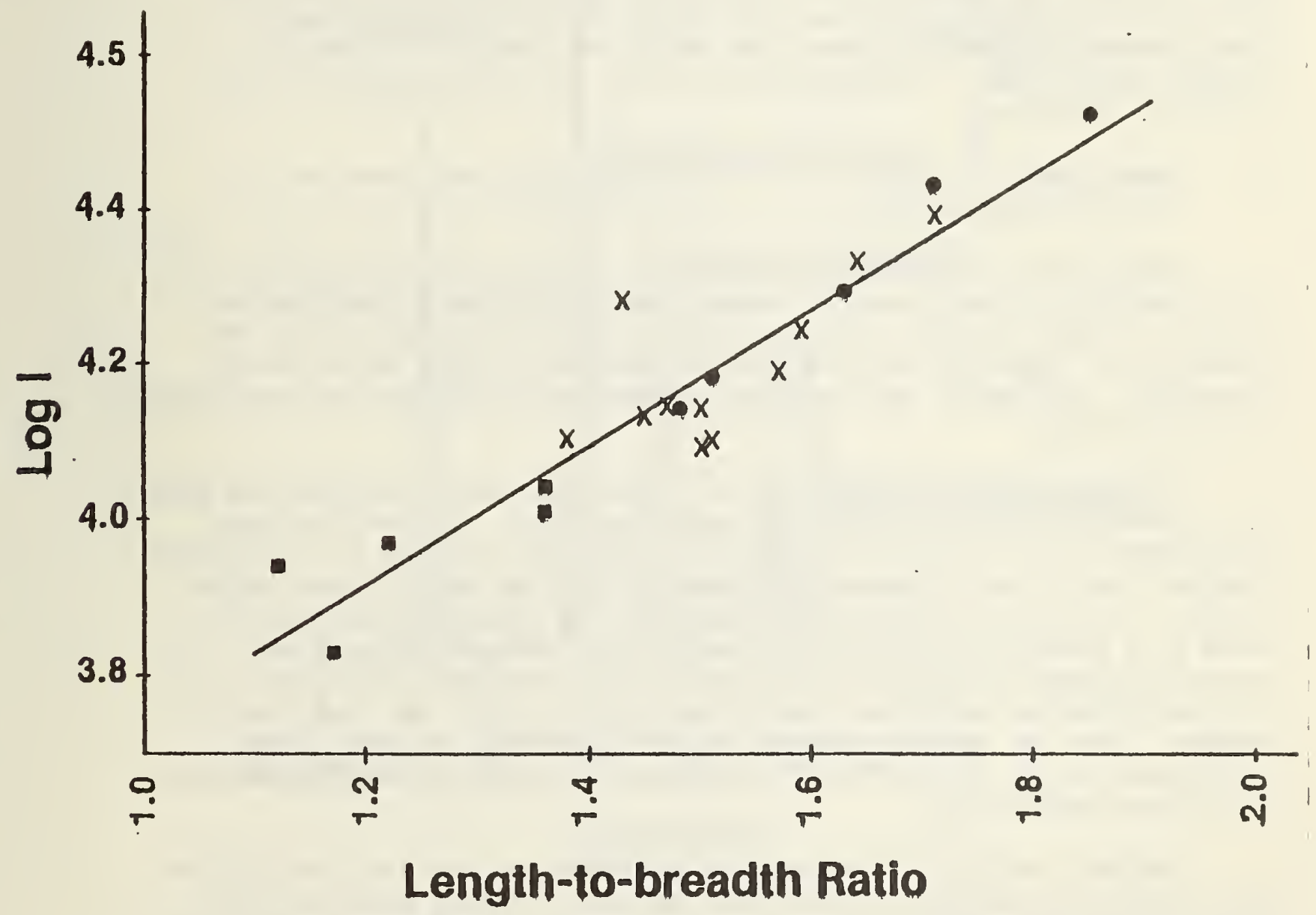

Figure 9. Linear correlation of LC retention data (Log I) and the length-tobreadth ratios for methyl benzo[c]phenanthrenes ( $\infty$ ), methyl benz[a]anthracenes $(X)$, and methyl chrysenes $(\bullet)$. 
cation was complicated by the formation of an electrode deactivating film of hexaphenylditin. This problem was overcome using pulsed detection wave-forms which take advantage of the electrochemical reversibility of the reduction (see figure 11 ).

A comparison has been made of several materials for the working electrode of the detector cell. A dropping and hanging mercury electrode cell, as well as several solid-electrode thin-layer cells (based on mercury-coated gold, platinum, and glassy carbon), were evaluated for their negative potential range and detection limits for reducible organic pesticides. A manuscript on LC-EC has been submitted to Analytical Chemistry.

\section{a. Development of a High-Sensitivity Dual-Electrode Detector for Liquid Chromatography}

Reductive electrochemical detection in liquid chromatography (LC) is potentially a very powerful measurement technique in the determination of trace organic pollutants by virtue of its sensitivity and selectivity. However, for the best sensitivity, oxygen exclusion is required. This makes the technique cumbersome to use and has restricted its application.

A new approach for reductive LC detection that eliminates the need for complete oxygen exclusion has been developed. A two-electrode, thin-layer cell is used in a manner conceptually similar to a ring-disc system. Two closely spaced electrodes are poised at different potentials. The first electrode encountered by the flowing electrolyte is held at a potential sufficiently negative to reduce the analyte. The second detector electrode is held at a potential sufficiently positive to reoxidize the generated species and also to avoid the reduction of oxygen. The low residual current at this potential assures a low background noise, upon which the analytical current is easily measured with high sensitivity.

The best analytical response is obtained for analytes that either are reversibly reduced or that have chemical followup reactions that generate easily oxidized products. The reductive/oxidative behavior of three important industrial/environmental pollutants - nitrobenzene, dichlone, and tributyltin - have been studied by reverse pulse voltammetry.

The sensitivity and linearity of the dual electrode technique has been evaluated by comparison to direct reductive detection. Two chromatograms with equal concentrations are pictured in figure 12. The baseline noise of direct reductive detection without use of an inert atmosphere box is apparent (upper figure). The lower chromatogram shows the very low baseline noise of the dual electrode approach achieved with a minimum of difficulty.

Current experiments involve the evaluation of the technique's selectivity by application to the measurement of organic analytes in environmental samples. Also, investigation is in progress on the use of modulated waveforms and phase selective detection to improve selectivity. 


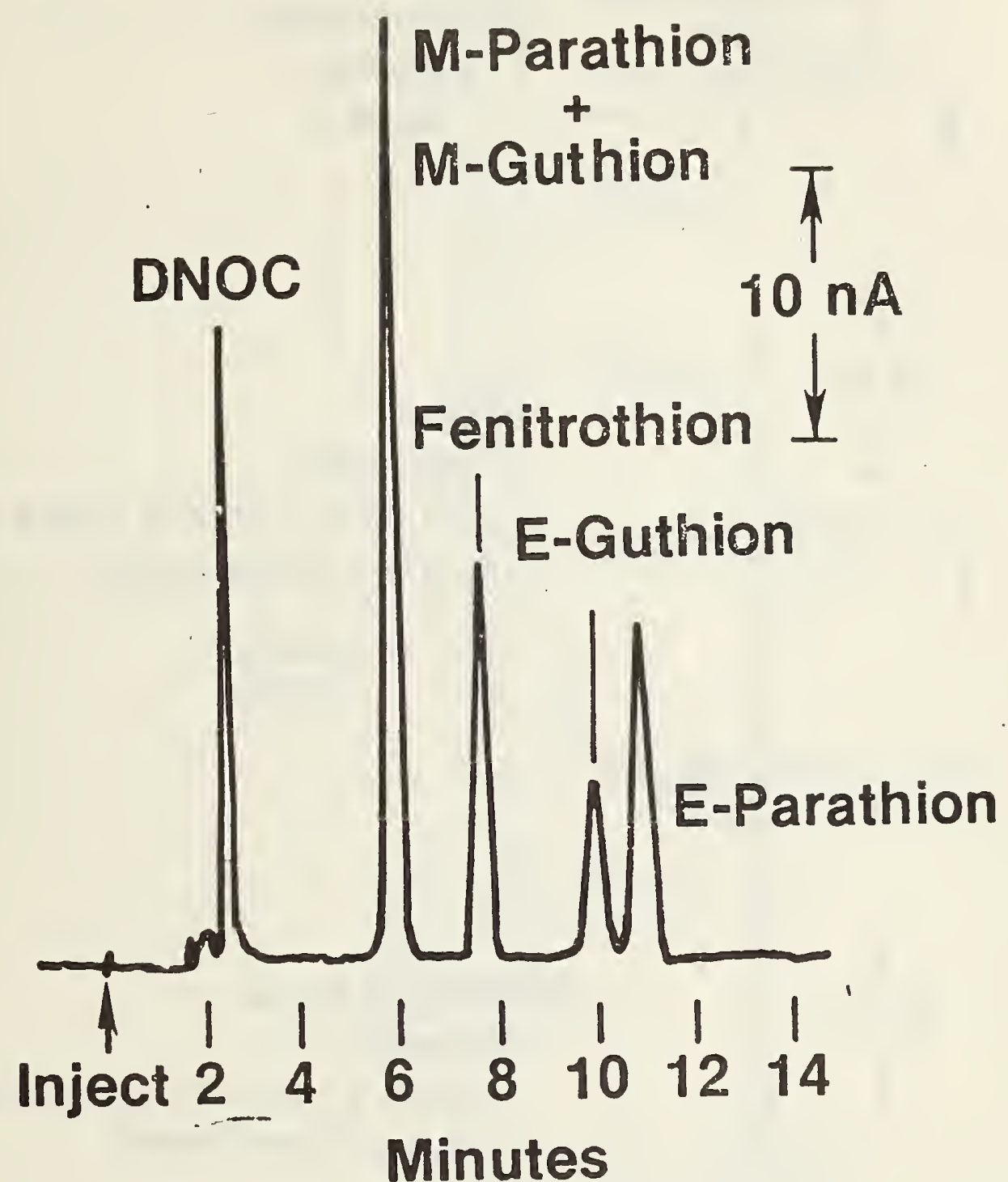

SEPARATION OF SOME INSECTICIDES

Column: Spherisorb ODS, $5 \mu \mathrm{m}, 4.6 \times 250 \mathrm{~mm}$

Soluent: $60 \% \mathrm{MeOH} / \mathrm{H}_{2} \mathrm{O}, 0.12 \mathrm{~mol} / \mathrm{L} \mathrm{NH}_{4} \mathrm{OAc}$

$1.5 \mathrm{~mL} / \mathrm{min}^{2}$

Detector: Amperometry $-0.85 \mathrm{~V}$, HMDE

Sample: $20 \mu \mathrm{L}$ of $10^{-5} \mathrm{~mol} / \mathrm{L}$ (about $3 \mathrm{ppm}$ ) of each

Figure 10. Reverse-phase insecticide separation employing amperometric detection. 

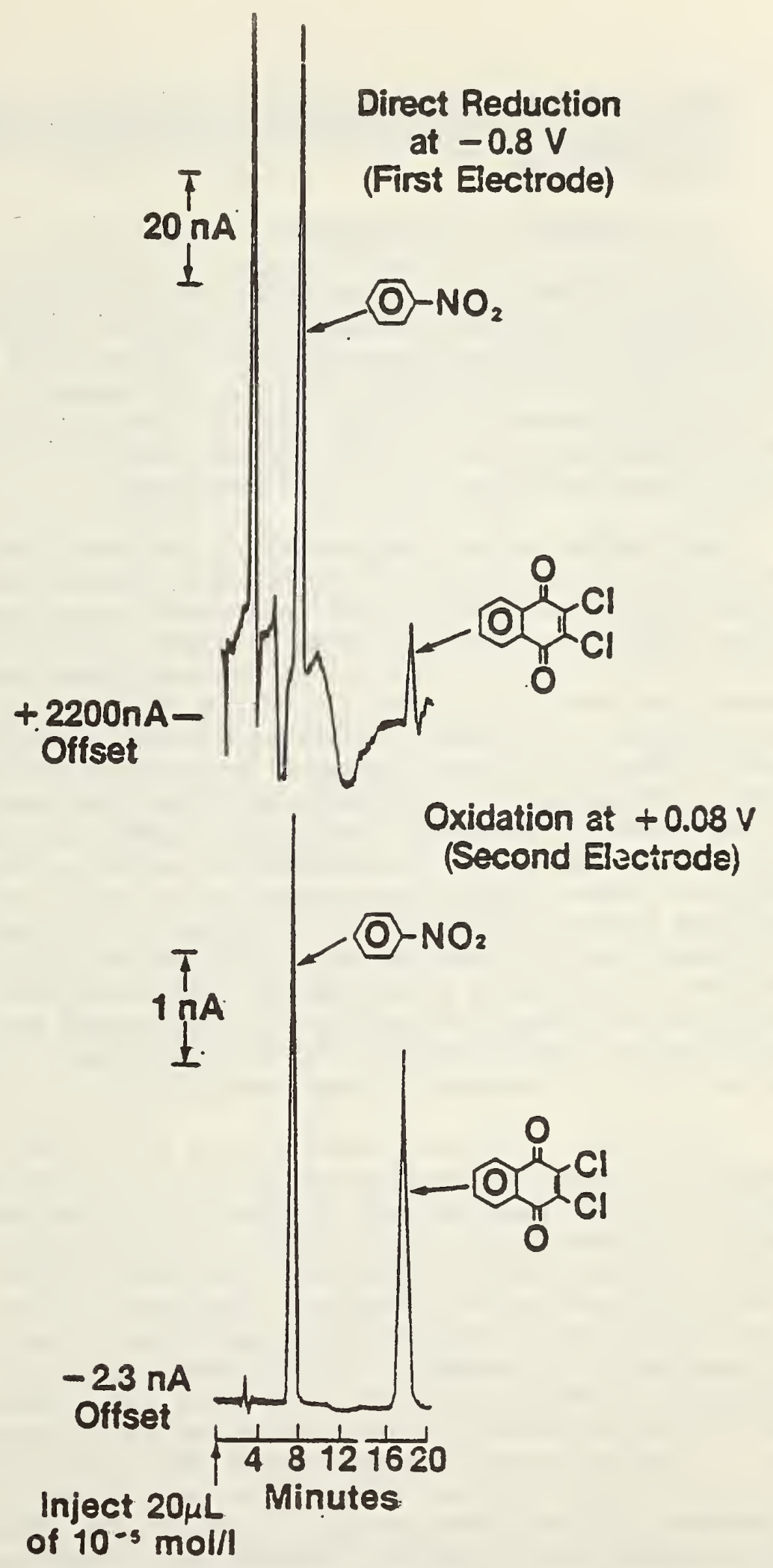

Figure 12. Different Reductive Detection Modes. Conditions: Analytes-$1.0 \times 10^{-5} \mathrm{~mol} / \mathrm{L}$ of nitrobenzene and dichlone; Solvent--60 percent $\mathrm{MeOH} / \mathrm{H}_{2} \mathrm{O}$, 0. $1 \mathrm{~mol} / \mathrm{L} \mathrm{NH} \mathrm{NH}_{4} \mathrm{OA}$ pH 5.2 at $1.0 \mathrm{~mL} /$ minute; Column--Whatman Partisil PXS $5 \mu \mathrm{m}$. Upper figure--direct reductive detection at $-0.8 \mathrm{~V}$ without enclosure of the LC in an inert atmosphere box. Lower figure--dual electrode detection, $-0.8 \mathrm{~V}$ on generator electrode, $+0.08 \mathrm{~V}$ on detector electrode. 
5. Development of a Fourier Transform Infrared Spectrometer-Gas Chromatograph Interface and Its Application to Pollutant Identification

\section{S. N. Chesler, F. R. Guenther}

Installation of a Nicolet 7000 Fourier transform infrared (FTIR) spectrometer was begun in September 1979, and essentailly completed by January 1980. The instrument was found to meet all design 'specifications and even exceed some, such as resolution and signal-to-noise ratio. The instrument is being used for four distinct research and/or service functions: high-resolution low-pressure gas work, liquid samples, liquid chromatography, and gas chromatography.

The studies of gas chromatographic applications have progressed through the utilization of packed column chromatography to the construction and testing of an interface for wall coated open tubular gas chromatographic columns (WCOT), which will allow real-time FTIR analys is of compounds eluting from the WCOT. The use of packed columns was not pursued because they were not found to have the resolving power required for the separation of the complex samples we wished to analyze. The WCOT columns used in this project were chosen to maximize sample capacity of the column, since the FTIR instrument requires $2700 \mathrm{ng}$ of component for the collection of a reasonably identifiable spectrum. Also, an on-column injector was fabricated to facilitate injection of a maximum amount of sample onto the column without undo loss of efficiency. The first interface design consisted of a Pt/Ir tube which connected the output of the thermal conductivity detector (TCD) to the FTIR light pipe. This interface was found to be unsuitable because too much dead volume (located mostly in the TCD and connecting fitting) caused unacceptably peak broadening. In addition, the Pt/Ir tube thermally trapped out and/or reacted with all solutes boiling above $300^{\circ} \mathrm{C}$.

The present interface consists of a $50 \mathrm{~cm}$ length of deactivated, methy 1 silicone-coated quartz capillary of $0.15 \mathrm{~mm}$ internal diameter. The TCD has been bypassed and no other gas chromatographic detectors are used. This interface is connected to a glass WCOT by use of AgCl cement; if a quartz WCOT were used, then the last $50 \mathrm{~cm}$ of the capillary itself could act as the interface. The quartz interface does not appear to react with or retard eluting compounds and spectra have been obtained for compounds such as methyl dibenzanthracene (b.p. $<500{ }^{\circ} \mathrm{C}$ ). Reconstructed gas chromatograms (from IR data) still show large losses in efficiency over those obtained from normal gas chromatographic detectors. This loss is most likely due to the large volume of the FTIR light pipe, and a new low volume light pipe is being procured. Even though the FTIR data does not fully reflect the high-efficiency separations obtained on the WCOT columns, very recognizable and identifiable spectra are being obtained from environmental samples being analyzed (e.g., phenols in shale oil, PCB's, etc.). This is, to a large extent, being made possible by some newly developed manipulative computer software which numerically regains some of this lost column efficiency. 
J. Stephenson, D. King, M. Jacox

Scientists in the NBS Center for Thermodynamics and Molecular Science (CTMS) have designed and completed the first stage of testing of a molecule specific measurement technique for the analysis of complex environmental samples for evaluation or compliance with EPA standards. In the past several years it have been recognized that realistic legislation impacting environmental quality must be targeted at a small number of potentially harmful chemicals, rather than gross regulation of, for instance, total organic carbon. Of the existing techniques, the computer controlled gas chromatograph-mass spectrometer system is the most powerful in terms of sensitivity and specificity. A high degree of sensitivity is required since many of these regulated chemicals have toxic or carcinogenic effects at levels in the ppt level; specificity is required since in many cases the toxicity of a given chemical species may vary as much as several orders of magnitude between isomers. This is particularly true for the dioxins.

The laser technique offers a quantiative sensitivity comparable to that obtained by state-of-the-art GC-MS systems, a much higher degree of selectivity, and should, when fully implemented, be suitable for certification of environmental SRM's and performance of referee-type measurements. This technique involves laser Raman scattering from molecules isolated in a cryogenic matrix. Raman spectroscopy provides a valuable fingerprint of the vibrational modes of the molecules present allowing for identification based on comparison to a library file of known spectra. Since the molecules are physically trapped in an inert matrix, there is no interference in the Raman spectrum of the first species due to the presence of a second or other species, the signals from a complex mixture such as expected from real environmental samples is simply the sum of the individual components.

To date, NBS scientists have successfully demonstrated the potential sensitivity and selectivity of this sytem in the analys is of laboratory samples of complex mixtures of volatile organic species at the sub nanogram level (corresponding to recapture of environmental samples at the ppt level). In these studies isomeric species were readily resolved with a $0.3 \mathrm{~cm}^{-1}$ resolution (isomeric peaks often show Raman shifts of $10-50 \mathrm{~cm}^{-1}$ ). The group recently acquired an LSI-11 controlled optical multichannel analyzer (OMA) and commercial gas chromatograph and have refabricated the sampling system to provide for higher sensitivity and sample thru-put and to allow analysis of real world samples.

The status of this work is now at the point that within 6 months our operative system should be ready for the acquisition, separation and analysis of environmental samples. Considerable help was received from colleagues at Argonne Laboratory. The initial tests of this system will include analysis of laboratory and real world samples of the tetrachlorodibenzo-dioxin isomers which cannot currently be distinguished by any existing technique at any concentration. 
(2) Methods Development for Inorganic Analysis

Participating Staff: Center for Analytical Chemistry

Background/Objective

In order to evaluate the environmental impact of expanded or new technologies, measurement techniques and monitoring systems are required in the development of a compatible data base to evalute the total effects of energy development upon our environment and to make rational policy decisons. Measurement techniques are either inadequate or do not exist to characterize and/or determine the low concentration levels of inorganic pollutants. Advanced measurement methods and instrumentation need to be developed to enable accurate measurement of energy related pollutants, as well as, data quality assurance of special field studies and analyses related to levels of pollutants injected into the environment.

The activities in this project focus on the development of measurement methods and instrumentation to enable the analysis of trace inorganic pollutants. Research efforts are also directed toward the development of measurement methods to distinguish forms (speciation) of inorganic pollutants in effluents in order to assess the transport, transformation and distribution of inorganic pollutants in the aquatic environment.

FY-1980 Milestones

- Complete trace elemental study of the 51 sampled sites throughout the Chesapeake Bay (top and bottom water column).

- Develop measurement methods to distinquish forms (speciation) of inorganic pollutants associated with energy related effluents.

\section{Selected Technical Highlights}

\section{Trace and Toxic Elemental Study of the Chesapeake Bay}

H. M. Kingston, R. R. Greenberg, E. S. Beary, T. C. Rains

As part of a multidisciplinary study of the Chesapeake Bay, the National Bureau of Standards (NBS) was asked to develop the techniques and procedures necessary to measure the trace and toxic element concentrations within the water column throught the entire length of the Bay. As part of this program, NBS collected 102 separate water samples and is currently completing the analys is for selected elements ( $\mathrm{Cd}, \mathrm{Ce}, \mathrm{Co}, \mathrm{Cr}$, $\mathrm{Cu}, \mathrm{Fe}, \mathrm{Mn}, \mathrm{Mo}, \mathrm{Ni}, \mathrm{Pb}, \mathrm{Sc}, \mathrm{Sn}, \mathrm{Th},{ }^{238} \mathrm{U},{ }^{35} \mathrm{U}$, and $\mathrm{Zn}$ ), including some elements at concentrations consistently below one picogram per milliliter (part per trillion). The collection of the samples was described in the 1979 Center for Analytical Chemistry Technical Activities, however, the list of elements to be analyzed was upgraded this year to its present complement with the addition of $\mathrm{Ce}, \mathrm{Sc}, \mathrm{Th}$, and $U$.

The literature on marine water analysis reflects the considerable difficulty in establishing an accurate and precise method of analysis for trace metals. An estuarine or sea water matrix defies a simplified approach. The extremely low trace concentrations cause the sample and 
analytical blank to be of paramount importance. The integrity of the sample can be compromised by just a brief exposure to normal laboratory air. In addition, the extremely high concentrations of alkali, alkaline earth, and halogen elements in the marine water matrix make direct analysis difficult or impossible for most analytical techniques.

To circumvent these problems, special chemical and instrumental procedures were developed. While the chemical separation and preconcentration was completed in late 1979, it was recently optimized for neutron activation analysis (NAA). The basic chemical procedure was developed and published in open literature in 1978 and 1979. The chemical procedure was substantially unchanged as reported for use with thermal device atomic absorption (TDAA). However, the elements were not eluted from the resin when used in conjunction with NAA. Both the modification and the established procedure proved to be extremely advantageous when applied to estuarine or seawater analysis.

The elimination of matrix elements allows the determination of many elements which could not otherwise be analyzed and enhances the sensitivity of other elements of interest. The control of the blank in this procedure has enabled its contribution to be sufficiently low that it does not limit the measurement of pristine samples. The particular fraction of each sample was prepared in the field by splitting a twoliter sample into two one-liter filter samples, one for TDAA and a second for NAA to eliminate handling and dividing a single particulate sample.

Both the samples and the blanks have undergone rigorous statistical scrutiny and are being reported after correction for blank contribution (where necessary) at the 95 percent confidence limit.

It was necessary to extend the capabilities of the chemical and instrumental techniques to obtain state-of-the-art concentration data for the samples. All of the elements of interest cannot be analyzed by a single instrumental technique. The methods necessary to analyze the estuarine and seawater using NAA have been developed this past year. The combined use of NAA and TDAA provides the capability to determine a wide range of important trace elements in estuarine samples. It was found that by drying the resin in the column after chemical separation and analyzing it directly in heat sealed polyethylene film that NAA could simultaneously analyze many elements at the ultratrace level with a high degree of accuracy. This detailed technique will be published in the open literature in the near future.

These concentration measurements will be used as the basis for several studies. Because the samples were taken concurrently with sediment, pore water, and benthic studies, both the water-to-sediment equilibrium and aquatic population will be correlated. Beyond this, a "state-of-the-Bay" is being established which includes an estimate of the impact of the population area impinging on it. The ultimate goal of the program is to produce an informative study to guide the management of the Bay. To meet these demands, it is necessary to compare the results respective of their location throughout the Bay. The interpretation of the concentration data obtained at the different locations 
throughout the Bay is extremely difficult. At a single location the concentration can vary with time, tide, current, wind conditions, temperature, biota, etc. With these perturbations of concentration at a single location, interpretation of the differences found at separate locations is formidable. One approach which has proven useful in minimizing fluctuations is the comparison of elemental ratios rather than absolute concentrations. For this technique to be successful, however, the elemental concentration must be determined with a high degree of accuracy. Reference elements must be chosen which are ubiquitous and essentially nonanthropogenic. An increase in the total mass loading will increase the absolute concentrations, but will not affect the elemental ratios. A significant addition of a single element or several elements to the environment from either natural or anthropogenic sources will increase the ratios of this (these) element(s) with respect to the reference element. Increases in concentration, however, may not be obvious over the normal fluctuations of the absolute elemental concentrations. This technique has been applied to the elemental concentrations in the estuarine samples with dramatic results. The elemental concentrations in the particulate fractions were found to vary over two orders of magnitude between locations as indicated in Table 15 . The ratios for $\mathrm{Fe}$, Th, and $\mathrm{Ce}$ are essentially constant, differing by only 20 percent, while the Co to Sc ratio is four times greater in sample 11,032 than in either 11,005 or 11,007.

Table 15. Concentration on Particulate Phase and Ratios to Scandium. Sample Number

Element 11,005 11,007 11,032

$\mathrm{Fe}$

Co

Sc

Th

Ce e Concentration $=-n \mathrm{~g} / \mathrm{mL}$

$\begin{array}{cll}18 & \pm 1 \\ 0.028 & \pm 0.003 \\ 0.0049 & \pm 0.0002 \\ 0.0038 & \pm 0.0005 \\ 0.039 & \pm 0.003\end{array}$

\section{Ratio to Scandium, $[X] /[\mathrm{Sc}]$}

$\mathrm{Fe}$

$3.2 \times 10^{3}$

$3.2 \times 10^{3}$

$3.7 \times 10^{3}$

Co

$$
1.1
$$

1.3

5.7

Sc

$\equiv 1$

$\equiv 1$

$\equiv 1$

Th

$\mathrm{Ce}$

6.7 
This technique provides a means for identifying the similarities and differences between the sample locations. A comparison of ratios throughout the Bay may be useful in identifying anthropogenic and/or naturally occurring sources to the Chesapeake Bay. If the concentrations of toxic elements are found to be excessively high, it is necessary to identify their sources before any attempt can be undertaken to reduce these levels.

\section{Sulfur Speciation in Coal}

\section{S. Hanamura}

This methodology is based on the measurement of the characteristic vaporization temperatures at atmospheric pressure of the various forms of sulfur, using plasma emission spectrometry to monitor each vaporized sulfur species. The resulting spectrometric thermogram consists of a series of relatively broad peaks, each peak being a different sulfur species.

For this experiment, the $1900.27 \&$ sulfur atomic emission line was used with a HTV R-919 photomultiplier tube and a one-meter monochromator with air purging. This system, using the 1900.27 \& line can detect 100 $\mu \mathrm{g}$ of sulfur. If air is purged and the $1826.25 \AA 1$ ine is used, the detection limit can be lowered to $5 \mu \mathrm{g}$. Previously, three sulfur species in coal have been reported in the literature, pyritic sulfur $\left(\mathrm{FeS}_{2}\right)$, sulfate sulfur $\left(\mathrm{FeSO}_{4}\right)$, and an "organic" sulfur. In the present experiments, sulfur and iron emission intensities were monitored as a function of temperature.

From these experiments, four sulfur species have been found, $\mathrm{FeS}_{2}, \mathrm{FeSO}_{4}$, organic sulfur, and a low evaporation temperature sulfur. This low evaporation temperature sulfur starts to evaporate at $28{ }^{\circ} \mathrm{C}$. SRM 1635 Western Coal has been found to contain about 18 times more of this species than SRM 1632a Penn Coal. Also, organic sulfur has been found to be linked with iron. In a previously described analytical technique, organic sulfur was calculated by substracting pyritic and sulfate sulfur from the total. Therefore, the value obtained from the "organic" sulfur species included the low evaporation temperature sulfur. In the future, an attempt will be made to identify the chemical forms of these species using thermal separation and a cold trap collection technique. Also, an investigation will be undertaken in which two monochromators are used simultaneously to detect both sulfur and iron or any other elements which may be bound with sulfur.

Sulfur species analysis still needs work on the development of a standardization procedure, but it is a simple technique and has potential for the direct determination of each species present. 


\section{Ionic Chromium Speciation Measurements}

J. D. Messman, T. C. Rains

An anion-exchange method has been developed for the separation of trivalent and hexavalent forms of chromium in synthetic aqueous solutions. The aqueous sample is made $\mathrm{IN}$ in $\mathrm{HCl}$ and is then introduced onto the anion-exchange column. In a $1 \mathrm{~N} \mathrm{HCl}$ medium, $\mathrm{Cr}$ (III) elutes from the column while $\mathrm{Cr}$ (VI) is adsorbed by the active sites of the anionexchange resin. After $\mathrm{Cr}$ (III) has eluted from the column, a solution of $1 \mathrm{~N}$ ascorbic acid in $1 \mathrm{~N} \mathrm{HCl}$ is added to the column which then reduces the $\mathrm{Cr}$ (VI) species to $\mathrm{Cr}$ (III) species directly on the column. The newly-formed $\mathrm{Cr}$ (III) species are then eluted from the column as in the first part of the procedure. Atomic absorption spectrometry is used for on-line detection of the chromium species as they elute from the column. The aspiration uptake capillary of the AAS nebulizer is replaced by a Teflon microsampling cup. The anion-exchange column is positioned so that the eluent drops directly into the microsampling cup for continuous nebulization and atomization of the sample. A chromatogram which illustrates the direct speciation of trivalent and hexavalent chromium by ion exchange-atomic absorption spectrometry (IE-AAS) is shown in Figure 13 . 


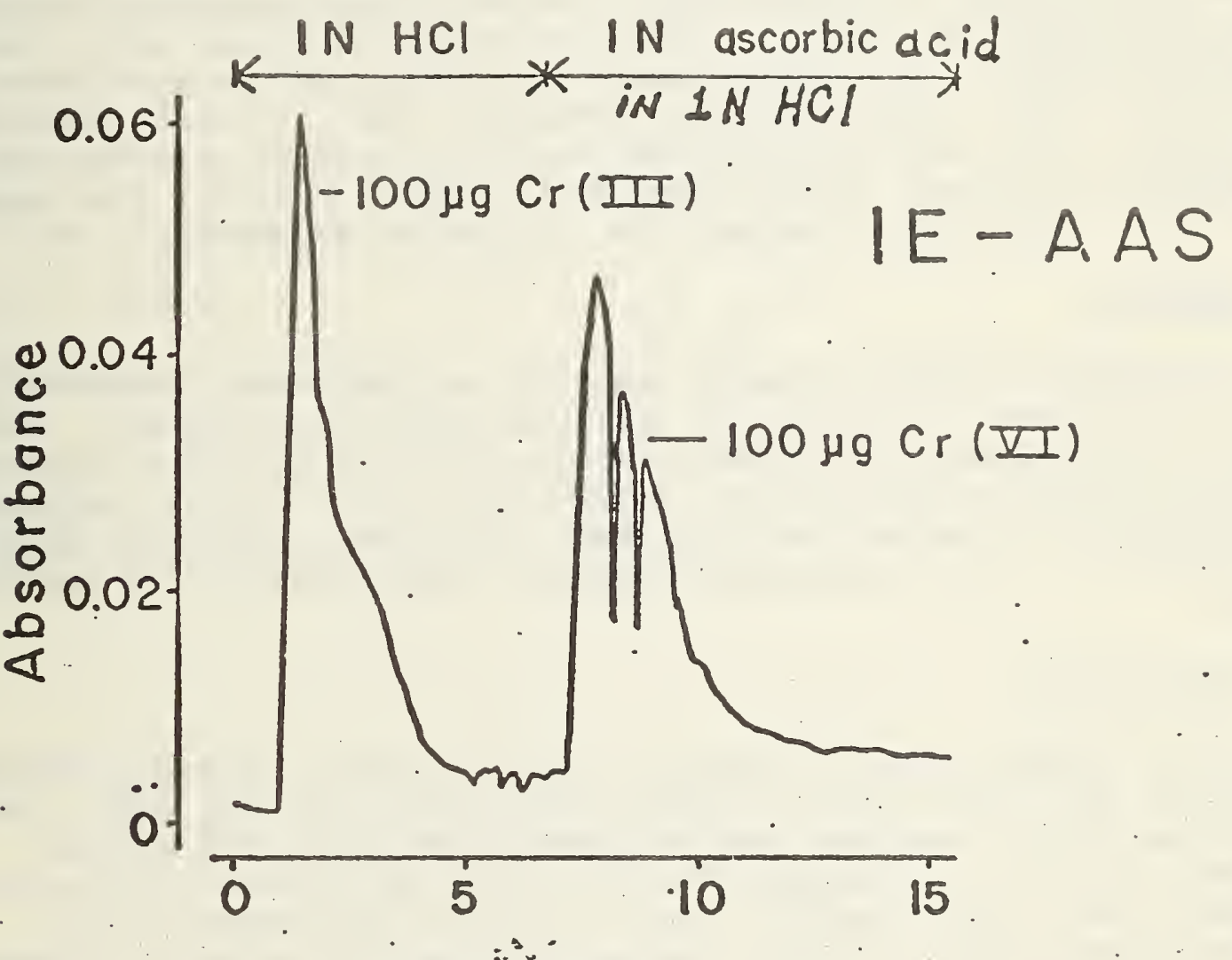

\section{Minutes}

Figure 13 


\section{a. Overview}

The national program for assessment and abatement of pollutant emissions to the atmosphere is predicated on concerns for the known or anticipated detrimental effects of such emissions on human health and the environment. National ?egislation mandates that emission assessments be performed by monitoring air pollutant concentrations from stationary and mobile sources. Such activities involve a broad spectrum of measurements carried out by municipal, state and federal agencies, and by the industrial sector. In order to assure the quality and intercomparability of data over this broad measurement conmunity, highly reliable standards are needed. This Task addresses the development of high priority research standards and Standard Reference Materials (SRII's) to assist attainment of national program data quality in air pollution monitoring.

\section{Objective}

To develop priority research materials and SRM's that provide data quality assurance in national air pollution monitoring programs. This Task covers the development of national standards for air pollution assessments required by current legislation. Activities in this Task are represented by topics such as ambient and stationary source gases, asbestos, urban air particulates, trace inorganic and organics in particulates, and organic gases.

\section{Interactions}

This program strongly interacts with the Office of Standard Reference Materials, OSRD, with various Centers (CAC, CTMS, CRR, CMS, CAM). The activities within this Task involve direct interactions between the involved technical and managerial staff of NBS and relevant staff complements within other agencies, such as the U.S. Environmental Protection Agency (under EPA/NBS Interagency Energy/Environ-mental Agreements). Strong interactions also exist with industry, state and local government agencies, research laboratories and universities. Related NBS R\&D activities regarding the improvement or innovation of methods having potential or actuai application to the establishment of Ril's or SRM's for data quality assurance of air pollutant measurements are closely followed.

\section{Task Justification}

Development of quality assurance reference standards is essential for valid air quality monitoring data to address the attainment of national goals for the public health and welfare, and for the concomitant assurance of a viable ecological environment. This program directly impacts the meaningful assessment of airborne pollutants and national air quality. The activities of this program are required, provided under, or have been recommeded by the. Clean Air Act, its 1977 Amendments, The Standing Air Monitoring Working Group, the Council on Environmental Quality, the Interagency Task Group on Environmental Data and Monitoring, the Toxic Substances Control Act, and the NBS Organic Act. 
b. List of Projects

Project Title Source of Funding*

A sbestos in Air

Toxic Organics - Air

Acid Precipitation Stds.

A ir Generator Columns

0 zone Photometers

Gas Standards

Particulate Standards
$P / T-E P A$

$P / T-E P A$

$P / T-E P A$

$P / T-E P A$

$P / T$ - EPA

$E / E-E P A$

$E / E-E P A$

*P/T: Pass Through - E/E: Energy/Environment 
(1) STANDARD REFERENCE MATERIAL AND REFERENCE METHOD FOR ASBESTOS IN AIR

Participants: John Sma11, Eric Steel, Patricia Johnson, Patrick Sheridan

Goa 1

To assist EPA in improving the reliability of measurements of asbestos fibers in air by providing standards for calibration and measurement and reference measurement technology.

\section{Objectives}

- Provide calibration standards to be used for the determination of relative sources of error in EPA's Analytical Electron Microscopy (AEM) provisional reference method, for laboratory and field evaluation of the reference method, and for interlaboratory collaborative testing.

- Provide a laboratory evaluation of key procedural variables in the provisional reference AEM method, and recommended optimized procedural parameters.

\section{Technical Approach}

The major objective of this program will be to develop and provide calibration standards consisting of asbestos fibers dispersed in an appropriate matrix and deposited on a filter substrated. These standards will be designed for use in a quality assurance program to improve and maintain the reliability of measurements of asbestos fibers in the ambient atmosphere. A secondary objective will be to utilize the competence and facilities at NBS to evaluate key procedural variables in EPA's provisional reference method.

The development of an asbestos standard of the quality and quantity required for an NBS certified Standard Reference Material (SRM) would require a considerable length of time. Research materials will be provided in a limited quantity in order to satisfy the interim requirements for conducting methods evaluation, field testing, and interlaboratory comparisons. The interim research materials, by definition, will not be NBS SRM's certified in terms of fiber count or homogeneity on the filter, but the fiber count and homogeneity data provided with the research material will be the best attainable within the time frame of the milestones in Part F. Limits of error will be provided by counting statistics for fiber count and degree of sample homogeneity. The research materials provided in FY-79 and early FY-80 will be assayed for fiber count only. Information on size distribution, and identification of fiber mineralogical group will be provided at a later date. The work performed in developing these research materials will provide the basis for the ultimate provision of NBS asbestos fiber SRM's. 
The limited amount of research materials provided during FY-79 will be developed by borrowing time on an AEM. An NBS dedicated AEM will be required in order to undertake a variety of activities associated with this program. Optimization of the AEM would require modification not permissible on a non-NBS system. The NBS AEM will be used to provide:

0

the inclusion of amphibole fibers with chrysotile, with quantitative information on both fiber types.

0 quantitative information on fiber size distribution.

${ }^{0}$ more substantial amounts of research material.

0 the ability to evaluated key parameters in the EPA AEM provisional method.

- a detailed examination of urban airborne particulate as a filter matrix.

${ }^{0}$ support analyses of filter samples for EPA.

The FY-79 program is summarized as follows:

- Counting grid standards for the AEM will be prepared and analyzed. These will be electron microscope grids on which NIEHS short chrysotile fibers have been dispersed in an appropriate matrix. These grids will be useful in evaluating the statistical counting portion of the EPA provisional method, and will provide a means for experimentally analyzing counting statistics and the ability of operators to distinguish fibrous versus non-fibrous material.

- In order to evaluate the complete procedure (including sample preparation), chrysotile fibers dispersed in a suitable matrix, preferably urban air particulate (UAP), will be deposited on Millipore or Nuclepore filter paper. Mechanisms will have to be developed to attain homogeneous dispersal and deposition of the fibers and the matrix on the filter. A statistically-designed plan will be developed in order to define the level of counting required for the determination of fiber density and homogeneity on the filter.

- NBS, in collaboration with EPA, will define and prioritize key procedural parameters in EPA's AEM provisional method that require laboratory evaluation and optimization. 
Description of Initial Project Activities

- Investigation of Mechanisms for Fiber and Matrix Mixing Dispersal, and Filter Deposition

The filter research material and the filter calibration material will requie a uniform deposit of chrysotile fibers and matrix material on a filter. A liquid suspension will be used, and a variety of liquids, surfactants, and agitation techniques will be investigated to determine the best combination for uniform deposition.

Two approaches to mixing the NIEHS chrysotile fibers with the matrix material will be tested. One method is to combine the two in a liquid suspension before filtering; another approach is to use a two-step filtration--first filtering a suspension of chrysotile, then filtering a suspension of matrix material, onto the same filter. Other parameters that may have adverse effects on the homogeneity of the deposit (such as erratic or high background levels of chrysotile from blank filters) will also be studied.

- Preparation of Asbestos/Carbon Grid Sample

The operator counting error needs to be determined statistically before a reference filter can be fully characterized. In order to do this, some of the preliminary filters developed in $A$. above wi11 be mounted on a TEM finder grid using a carbon coating and the Jaffe wick filter dissolution technique. Three concentrations will be used to mimic the range of conditions found in typical samples.

The loadings will be approximately 5-10 fibers per grid-square, 25-50 fibers per grid-square, and over 100 fibers per gridsquare. These grids will be counted by several operators, and the fiber count for each marked grid-square will be recorded. Statistical evaluation of these data will be used to determine operator variance.

- Preparation of Asbestos/Matrix Research Material

The knowledge gained in the previous steps will be combined to prepare a series of filter research materials with low, medium, and high loadings, having a defined distribution of fibers deposited onthe filters.

O Evaluation of the EPA AEM Provision Method

In collaboration with EPA personnel, we will identify critical variables in EPA's provisional AEM method which need laboratory evaluation. This evaluation will begin once the NBS AEM is operational. 


\section{Project Milestones and Estimated Completion Dates}

1. Complete counting grids research materials: NIEHS short fiber chrysotile in unambiguous synthetic matrix $\left(\mathrm{CaF}_{2}\right)$. (Completed)

2. Initiate identification of key parameters in AEM provisional method for detailed evaluation. (This milestone was initiated $6 / 79$ and will continue throughout program).

3. Install and accept NBS AEM system. (Completed for lst phase of program on chrysotile).

4. Complete counting grids research material: NIEHS short fiber chrysotile in a particulate matrix (UAP) - fiber count. (Completed - additional grids will be delivered to RTP as they become available).

5. Complete NBS AEM analysis of Urban Air Particulate (UAP) for use as a certified matrix. (Completed for phase 1 (chrysotile) of program; characterization of amphibole and other fibers is continuing).

6. Analyze up to a maximum of 50 filter samples (1ight to medium loading) for EPA using EPA and/or NBS analysts.

To be determined

$9 / 80$

$10 / 80$

9. Complete two Nuclepore "analyzed" filters with a light loading of chrysotile in UAP matrix (20-40 fibers/grid opening).

10. Complete two Nuclepore "analyzed" filters with a medium loading of chrysotile in UAP matrix (40-70 fibers/grid opening).

11. Report on summary of recommendations developed from NBS/EPA Asbestos Standards Workshop (held at NBS, 10/80).

$2 / 81$

12. Complete NBS AEM analysis of Urban Air Particulate for use as a matrix for the Characterization of amphibole fibers. 
13. In cooperation with EPA conduct an intermediate round-robin study making use of the "analyzed" filters from milestones 9 and 10 .

14. Initiate a proposal to Office Standard Reference Materials for the issuance of asbestos Research (RM) or Standard Reference Materials (SRM). RM/SRM to contain chrysotile in UAP matrix.

15. Develop the set of materials to be used for the RM/SRM.

16. Perform measurements leading to certification of RM/SRM.

17. Complete two Nuclepore "analyzed" filters with a light loading of a mixture of amphibole and chrysotile asbestos in UAP matrix.

18. Complete two Nuclepore "analyzed" filters with a medium loading of a mixture of amphibole and chrysotile asbestos in UAP matrix.

19. Complete counting frid research material. NIEHS short-fiber chrysotile in a particulate matrix (UAP) - fiber count. Deliver up to 10 additional grids.

20. Complete amphibole counting grid research material, crocidolite or Amosite asbestos in UAP matrix, to be used for fiber count and identification. Up to 10 grids to be delivered.

21. Issue special publication as a result of NBS/EPA Asbestos Standards Meeting.

22. Issue RM/SRM Nuclepore filters containing chrysotile in UAP matrix.

23. In cooperation with EPA conduct an intermediate round-robin study making use of the filters from milestones 17 and 18 .

24. Initiate a proposal to Office of Standards

Reference Materials for issuance of asbestos

RM/SRM. (amphibole/chrysotile mixture)

25. Develop the set of materials to be used for RM/SRM. (amphibole/chrysotile mixture) 
26. Perform measurements leading to certification of RM/SRM mixture.

27. Issue RM/SRM Nuclepore filters (mixture).

28. Perform work necessary to issue heavy loading filters greater than 70 fibers/grid opening.

29. Complete evaluation of EPA provisional method.

30. Conduct round-robin evaluation of EPA provisional method making use of the certified materials.

\section{Project Progress - FY-1980}

\section{(a) Project Synopsis:}

Initial work in FY-1980 focused on chrysotile asbestos fibers on filters, evaluation of the NBS Urban Air Particulates SRM (\#1648) as a useable urban air particulate matrix, and to various aspects of progress working with fiters. In corrdination with the NBS Center for Applied Mathematics, we analyzed counting data, representing approximately 150 grid squares at about 40 fibers per grid square. With this information, assessments were made of the operator error associated with measurements of asbestos on filter samples. This assesssment is critical to determining the level of accuracy with which a fiber concentration on a research material filter can be reported. In addition, fiber counting is a major parameter of the EPA Provisional Method for asbestos in air measurements under collaborative evaluation with EMSL/RTP. Preliminary studies of the potential loss of small fibers using the Provisional Method's $0.4 \mu \mathrm{m}$ filter relative to a $0.1 \mathrm{~m}$ filter, and homogeneity evaluation of fiber deposition on Nuclepore filters, were also carried out.

Work has progressed well on the preparation and counting of chrysotile asbestos fibers in an urban particulate matrix. It was determined that the NBS Urban Particulates SRM (SRM \#1648) was a usable air particulate sample for this purpose. In addition, counting grids of chrysotile asbestos in a fluorite matrix were forwarded to EMSL/RTP for distribution to non-EPA laboratories for evaluation. Further, deposition homogeneity of chrysotile asbestos in an urban air particulate matrix on Nucleopore filters was evaluated. Finally, a workshop, titled "NBS/EPA Asbestos Standards Workshop," was held at NBS October 1-3, 1980, jointly sponsored by EPA and NBS. Announcement letters were sent out to approximately thirty-five individuals involved in asbestos analysis from a list jointly compiled by EPA and NBS.

Data were collected on operator error and filter homogeneity for prepared samples of chrysotile asbestos in the Urban Particulates SRM (\#1648) deposited on a $0.4 \mu \mathrm{m}$ nuclepore filter. Histograms of asbestos fiber counts appears to fall between a normal and a uniform distribution for a given filter. A geographic analysis of the fiber distribution on 
the filters was carried out. The errors of five operators at NBS are now reasonably well defined, and usually fell in the $\pm 5-10 \%$ relative error range. Further definition of this error was possible using a device that allows the position of each fiber to be determined. Three counting grids were delivered to M. Beard (EMSL/RTP) in April. Interlaboratory error studies were initiated using these counting grids. Characterization of particulates in SRM 1648 continued. Speakers for specific review papers were invited to the asbestos standards workshop (see $(c)$, below).

A manual $x-y$ coordinate system employing digital voltmeters for mapping fiber location within a grid square was completed. The data collected with this system are used to plot locations of fibers counted by various operators. Operator countings are then correlated with respect to fiber locations and verification as chrysotile. The values are used to determine a verified count for a given grid opening. Plots are used to quantitatively and accurately compare operator counting abilities. Fiber counts on 38 grid openings, representing areas selected from the major portion of a filter surface, are under analysis by the NBS Center for Applied Mathematics. Preliminary results have indicated fiber distribution can be described by a Poisson distribution. Final planning is underway for distribution of Research Material filter segments to several laboratories for analysis. A round-robin analys is involving a number of principal asbestos analysis laboratories has also been planned.

(b) Evaluation of Counter Error in the Development of Asbestos Standards for the Electron Microscope - Interim Report

The Environmental Protection Agency has been monitoring the concentration of asbestos in ambient air, because of its potential adverse health effects, using an electron-microscopic anal of filtered air particulates. During a number of interlaboratory studies, EPA found that the reported asbestos concentrations varied among laboratories by up to five orders of magnitude on the same filter. Many laboratories consistently reported relatively high or low results, in part due to operator bias or error, filter inhomogeneity and counting statistics. Generally, because of the high magnifications used during analysis, only approximately $1 / 100,000$ of the total filter area was observed. The data from this small area were then extrapolated to the whole filter to determine the relative concentration.

Because of these problems with asbestos analysis, EPA requested NBS to prepare filters with known asbestos fiber concentrations that are homogeneous on the level of a $\mu^{2}$. In attempting to approach as closely as possible the true asbestos fiber concentration on the filter NBS has used up to five microscopists to analyze the same areas of filters. These areas were then sent to other experienced laboratories for analysis. The results are shown in Table 16. The variability of these counts both within NBS and at other laboratories was considered excellent by EPA. 
But these results could not be correlated to determine where the errors in analysis occurred. Also, it was felt that an average or mean value as reported in column 1 of this Table for the five NBS operators was not necessarily an accurate description of the number of fibers on the areas counted. For these reasons a digital specimen positioner was designed at NBS so that individual fibers could be uniquely identified by their $X-Y$ coordinates. Three to five NBS operators counted the same $75 \mu \mathrm{m} \times$ $75 \mu \mathrm{m}$ area, recording each fiber's $X-Y$ position. The counts were then correlated, and finally the area was reanalyzed to verify all fibers. In this way it was felt that the verified fiber count was as close as possible to the true concentration. Table 17 shows some of the data recently collected using this method. This Table shows each operator's error as number of fibers counted and how many of these were incorrect counts, how many fibers were missed, and the verified concentration.

Knowing the error associated with the five NBS operators and using the verified fiber counts has allowed work to proceed in characterizing fiber distribution and concentration towards a final product, an asbestos filter standard.

\section{(c) NBS/EPA Asbestos Standards Workshop}

Following is a listing of the Workshop program. A questionnaire has been developed on the Workshop and sent to participants in order to develop the final Workshop recommendations. These finalized recommendations will be submitted to EPA for review and consideration. A published Proceedings of the Workshop is planned, covering the full manuscripts of the twenty-three talks presented.

\section{Introduction:}

"Quality Assurance for Airborne Asbestos Measurements" M. Beard (EPA)

Session I - Bulk Materials for Preparation of Asbestos Standards:

"Asbestos Reference Materials: Sources and Characterization" -- R. Draftz (IITRI)

"Glass as a Material for Asbestos Standards"

D. Cronin (NBS)

"NBS Standards Program"

K. Kirby (NBS)

"Analysis of NBS SRM \#1648 for Use as a Matrix Material

in the Development of Asbestos Filter Standards"

J. Smal1 (NBS)

"Bulk Standards and Their Relationship to Polarized

Light Microscopic Analysis of Asbestos Containing

Materials" -- R. Virta (BOM)

"Preparation of Asbestos Standards for Methods Vertification and Laboratory Evaluation" -- D. Lentzen (RTI) 
Session II - Standards Preparation:

"Sample Preparation for Quantitative Electron

Microscope Analysis of Asbestos Fiber Concentrations"

P. Cook (EPA-Duluth)

"Development of a Waterborne Asbestos Reference Material"

R. Feldman (EPA-Cincinnati/University of Virginia)

"Preparation of Air Standards"

D. Jones (IITRI)

"Concentration and Separation of Chrysotile by Two Phase Liquid Separation"

C. Melton (Battelle)

Session III - Asbestos Analysis for Standard Certification:

"Analytical Procedures and Standardization for Asbestos Fiber Counting in Air, Water, and Solid Samples"

E. Chatfield (ORF)

"Automated Particulate Measurements by Image Analysis" R. Clark (MSHA)

"Development of a Rapid Survey Technique for the Detection of Asbestos Fibers in Water Samples"

P. Ri is (ORF)

"Interlaboratory Measurements of Amphibole and Chrysotile Fibers in Water"

K. Chopra (Union Carbide)

"Low Fiber Concentration Counting Difficulties"

R. Lee (U.S. Steel)

Session IV - Error Analysis and Statistics:

"Membrane Filter Method: Statistical Consideration"

G. Chase (Johns-Manville)

"Simulation of the EPA Provisional Method for Airborne Asbestos Concentrations"

T. Fitz-Simons (EPA)

"Minimization of Operator Error in AEM Analysis of Asbestos" -- E. Steel (NBS) 
"Asbestos Filter Counting and Homogeneity Error Analysis and Statistics" - S. Leigh (NBS)

Session V - EPA Provisional Method:

"Refinements in the EPA Provisional Methodology" G. Yamate (IITRI)

"Fiber Identification and Blank Contamination Problems in the EPA Provisional Method for Asbestos Analysis"

S. Ring (Minn. Dept. of Health)

"MSHA Standard Method for Fiber Identification by Electron Microscopy"

R. Clark (MSHA) 
Table 16. A Comparison of Asbestos Fiber Counts from Four Laboratories for Nine Areas. (Number of Fibers per $\sim 75 \mu \mathrm{m} \times 75 \mu \mathrm{m}$ area).

$\begin{array}{ccccc}\text { Area } & \begin{array}{c}\text { Laboratory (NBS) } \\ \text { (Five Operators) }\end{array} & \underline{(2)} & \underline{(3)} & \underline{(4)} \\ 1 & 32.6 \pm 2.5 & 23 & 42 & 22 \\ 2 & 31.8 \pm 1.6 & 32 & 36 & 33 \\ 3 & 27.6 \pm 1.5 & 22 & 23 & 22 \\ 4 & 15.8 \pm 1.5 & 16 & 13 & 15 \\ 5 & 49.8 \pm 4.4 & 33 & 32 & 40 \\ 6 & 51.0 \pm 2.3 & 37 & 40 & 45 \\ 7 & 53.2 \pm 3.7 & 35 & 39 & 44 \\ 8 & 53.0 \pm 3.5 & 33 & 38 & 42 \\ 9 & 39.0 \pm 1.6 & 22 & 30 & 40\end{array}$

Table 17. Operator Error Data for a $75 \mu \mathrm{m} \times 75 \mu \mathrm{m}$ Area Using a Verified Counting Technique Based Upon an NBS Designed Speciman Position Digital Readout.

Operator

Total Fibers Counted

31

24

20

25
Fibers Not
Counted

2

2

8

1
Fibers Incorrectly
Counted

7

Verified Fibers Counted

26

26

26

26 
(2) STANDARD REFERENCE MATERIALS (SRM'S) FOR TOXIC ORGANICS IN AIR

Participants: W. Schmidt, W. Cuthrel1

Background

The Toxic Substances Control Act of 1976 focused attention on the need for a better understanding of the effects of chemicals on human health and the environment. It has been estimated that 70,000 chemicals are currently in use. Although only a small number of these are toxic, the risk to human health and the effect on the environment will be the subject of intensive study during the next five years. Many classes of compounds will be scrutinized by the EPA. One of the largest classes of compounds will be organics, which are used widely in industry as precursors in the manufacture of synthetics and as solvents in various commercial operations. To identify and measure the concentration of organics present in the atmosphere, the Environmental Protection Agency is involved in monitoring programs for organic compounds. It is expected that the number of such projects will increase.

Essential to a successful monitoring activity is availability of certified standards materials in the measurement range of interest. These standards are needed for calibration of the measurement technique and in quality assurance activities necessary to determine the precision and accuracy of the monitoring data gathered. Organic standard materials having certified concentrations of organics are not available for use with air monitoring methodology. Since the need for standard materials will increase with additional monitoring activity, development for organic Standard Reference Materials should be initiated. The logical area for this effort is the National Bureau of Standards (NBS). NBS already produces Standard Reference Materials (SRMs) of many and varied types, and has the facilities and expertise needed. SRM's produced by NBS are characterized over long periods of time before they are added to the catalog of materials available. Research materials (RM's) are also available from NBS, which have been carefully analyzed, but are not as extensively characterized as SRM's. New organic RM's would meet the more immediate monitoring needs of EPA. 
The immediate purpose of this Task is the development of organic RM's by NBS for use by EPA in their monitoring and quality assurance programs. NBS development of SRM's rather than RM's will be considered separately for each case as needs become identified during the course of this multi-year program, following EPA and NBS concurrence. These materials will include toxic organic compounds in compressed gas cylinders, and in permeation tubes. Analysis is needed on special high priority materials, such as permeation tubes, cylinders gases, substrates with volatile organics deposited on them, and for flow measurement. In conjunction with the preparation of toxic gas RM's, toxic gas analytical methods research will be carried out by NBS to evaluate appropriate methods or devices for toxic gas standards. NBS will also pursue an initially modest effort in the characterization and quantification of polynuclear aromatic hydrocarbons on urban particulate dust.

\section{Selection of Organic Compounds}

Lists of organic chemicals to be tested for toxicity and impact on the environment are being updated by EPA based on new information as it is gathered. Three EPA lists of toxic chemicals are presently available: one from the Office of Toxic Substances; one from the Office of Air Quality Planning and Standards; and one based on ambient air organic screening projects carried out by EPA. A number of gaseous toxic organic compounds are common to all lists. Of this number, the following are considered by EPA as most compatible with development as NBS RM's in cylinders or permeation tubes: toluene, chloroform, trichloroethylene, carbon tetrachloride, ethylene dibromide, ethylene dichloride, benzene, and perchloroethylene. Research materials containing lower volatility materials, including benzo[a]pyrene, biphenyl, and anthracene, are also of interest to the EPA.

\section{Research Materials (RM's)}

Research Materials of the permeation tube or compressed gas cylinder type will be provided for generation of known gas concentrations in the range of interest. A teflon permeation tube filled with an organic compound allows for generation of a range of gas concentrations if the permeation rate of the tube is known. The permeation rate of the tube is dependent on a number of factors, including the physical properties of the permeant, the temperature of the permeation tube, and the thickness of the tube. Permeation tubes have been successfully prepared by EPA for perchloroethylene, ethylene dichloride and benzene. It is likely that permeation tubes can also be sucessfully prepared for the other volatile compounds listed in the preceding section. Permeation rates of interest to the EPA are in the range of 100 to $1000 \mathrm{ng} / \mathrm{min}$ at $25^{\circ} \mathrm{C}$. A determination whether permeation tubes can be prepared for each compound, and subsequent tests for their stability are necessary initial steps in their certification as RM's. If permeation tubes cannot be successfully prepared for some of the organic compounds, other approaches wi11 be considered. 
Compressed gas cylinders also provide a convenient method for providing gases in concentrations needed for calibration. The success of this approach is dependent on, among other factors, the reactivity of the gas with the cylinder wall, regulators and makeup gas. Concentrations of some gases in cylinders listed in the above section have been successfully prepared in the range of $10 \mathrm{ppm}$, which is the upper concentration range of interest. The long-term stability of these organic compounds in cylinders at the $10 \mathrm{ppm}$ range, and below, has not been determined conclusively. It is important to ascertain the stability of such organic gases at concentrations in the 5 to $10 \mathrm{ppm}$ range, as well as the feasibility of preparing stable compresseed gas cylinders in the $0.5 \mathrm{ppm}$ to $1 \mathrm{ppm}$ range. Some data exists to indicate that benzene and perchloroethylene have at least short-term stability in cylinders. The first two volatile organics to be examined as to feasibility will be benzene and perchloroethylene, since these are representative organic compounds of the volatile aromatic and halogenated organic toxic materials, respectively, of immediate interest in this Task.

Gaseous organic compounds deposited on air sampling substrates such as Tenax or charcoal will be necessary for use as quality control blind audit samples for EPA field monitoring studies. The long-term stability of these spiked tubes is doubtful. EPA may request NBS to serve as a reference laboratory to analyze spiked samples prepared by EPA. Such special analyses would fall under NBS Project (a) (Section C, below). Percent recovery of organics spiked on substrates has not been clearly defined for most of the organics listed and this problem will require resolution.

\section{Measurement Capability}

A measurement technique will be necessary for each organic compound listed. Fortunately, gas-liquid chromatography using a flame ionization (FID) or electron capture (EC) detector may be used to quantitate many organics. This analysis capability along with more sophisticated techniques not applicable as field methods will be used by NBS to determine the concentrations of organics in Research Materials. Analysis for organic compound impurities could be provided by gas chromatography coupled to mass spectrometry.

The Quality Assurance Divison of EMSL is setting up a gas chromatograph with FID and EC detectors. This chromatograph will be used in preparation and analysis of blind audit samples for use in field monitoring projects. Once this EPA project and the NBS effort becomes operational, an exchange of spiked samples and working standards may be made. 
Project Milestones and Estimated Completion Dates

(a) Analysis of Special Materials

- Evaluate current NBS analytical methodology for toluene chloroform, trichlorethylene, carbon tetrachloride, ethylene dibromide, ethylene dichloride, benzene, and perchloroethylene and report to EPA. (Completed)

0 Procure organics, analyze for purity and prepare primary standards and standard mixtures. (Completed) 7/79

- Analyze volatile organics in liquid and gas samples; check analysis method for linearity of response, precision, and accuracy. (Completed)

${ }^{0}$ Analyses of test samples provided by EPA. (On-going) FY 79/80/81

- Conduct reference analysis of special samples containing toxic organic gases in cylinders; as requested. (On-going)

(b) Development of Toxic Gas Standards

- Complete evaluation of permeation tubes and gas cylinders for benzene and perchloroethylene. (Completed) 5/80

0 Obtain permeation tubes for 6 remaining compounds . (Completed)

- Prepare $\mathrm{CCl}_{4}$ and toluene gas cylinders. (Completed) 6/80

- Initiate study of feasibility of permeation tube and gas cylinder standards for acrylonitrile. (Completed) $7 / 80$ 
- Complete ruggedness testing on permeation tubes and cylinders for long-term stability of benzene and perchloroethylene. (Completed) 9/80

- Complete analytical certification of benzene and perchloroethylene. (Completed) 9/80

- Initiate final analysis of benzene and perchloroethylene as RM's. (Completed)

$10 / 80$

- Complete evaluation of permeation tubes and gas cylinders for $\mathrm{CCl}_{4}$ and toluene for stability as RM's.

$12 / 80$

- Issuance of benzene and perchloroethylene as RM's. 9/81

- Complete evaluation of permeation tubes and gas cylinders for acrylonitrile and chlorobenzene.

$10 / 81$

(c) Toxic Gas Analytical Research

- Investigate absolute UV photometry for the determination of benzene in primary gas standards. (Completed)

$9 / 79$

- Initiate evaluation of Tenax/charcoal for benzene and perchloroethylene (adsorption efficiency, break-through rates, recovery, stability).

(Completed)

$6 / 80$

- Complete evaluation of UV photometric method for the analysis of trace benzene in air $/ N_{2}$.

- Report on initial experiments involving Tenax/ charcoal for benzene and perchloroethylene.

$9 / 81$

(d) Organics on Particulates

- Extensively characterize St. Louis Urban Dust SRM for polynuclear aromatic hydrocarbons. (Completed) 7/79

- Examine different extraction methods for quantitative removal of organic compounds sorbed on particulates. (Completed) 
Project Progress - FY-1980

\section{General Progress Synopsis:}

A set of six benzene and six perchloroethylene permeation tubes were equilibrated and calibrated gravimetrically at $25^{\circ} \mathrm{C}$. The benzene tubes were also intercompared using gas chromatography (GC) with flame ionization detection. A GC comparison of an EPA benzene tube with a calibrated benzene tube indicated the feasibility of this comparative technique for short term calibrations of benzene tubes submitted to NBS by EPA. Preparation of initial benzene and perchloroethylene gas mixtures in cylinders was also been completed. A report on the characterization of polycylic aromatic hydrocarbons on urban particulates and on evaluation of extraction procedures was sent to EPA.

A set of four gas cylinder research standards of benzene in air was prepared gravimetrically. These standards were compared with a benzene in air gravimetric standard prepared in May 1979, using gas chromatography with flame ionization detection (CG-FID). A least squares fit of the data yielded the following:

$\begin{aligned} & \text { Cylinder } \\ & \text { Number }\end{aligned}$
FF6976 (May 1979)
$X-207055$
$X-207094$
$X-207070$
$X-207099$

Gravimetric Concentration

$46.3 \mathrm{ppm}$

10.4

10.2

5.50

4.87
GC-FID

Analyzed

Concentration

$46.3 \mathrm{ppm}$

10.4

10.2

5.47

4.93

In addition, six permeation tubes of each of six of the other organics identified by EPA for this project were obtained. Gravimetric calibration of these permeation tubes at $40^{\circ} \mathrm{C}$ was also undertaken.

Evaluation of permeation tubes for benzene and perchloroethylene was completed, as well as evaluation of benzene gravimetric (gas cylinder) standards for linearity of response. Perchloroethylene gravimetric standards were prepared and evaluated. In addition, specially-treated aluminum cylinders were obtained and evaluated for their ability to maintain sample integrity. A special inlet unit was obtained for flash heating adsorption tubes for gas chromatographic analyses and a permeation tube dilution system was developed for generating known concentrations of organics. Also, gravimetric standards were prepared and are under evaluation for the evaluation of four mulitcomponent mixtures, as requested by EMSL/RTP. 
A sample of acrylonitrile has been obtained and planning for preparation of gas cylinder standard is underway. Long-term stability of benzene and perchloroethylene gas cylinders (6 mos) and permeation tubes $(1 \mathrm{yr})$ has been demonstrated. Temperature change effects will be examined as part of the ruggedness testing of these standards. Comparison of permeation tubes with gas cylinder standards for benzene and perchloroethylene indicate a correlation with less than $1 \%$ disagreement. Intercomparisons of the gravimetric cylinder standards shows an uncertainty of $20.5 \%$ relative. Multicomponent mixtures have been prepared gravimetrically and are being used to develop G.C. separation procedures as well as to determine the accuracy of the gravimetric standards procedure. A detailed FY-1980 progress report on this project is being prepared for EPA.

\section{Standards for Measurement of Benzene and Perchloroethylene in Ambient Atmospheres}

The first set of stable gas mixtures containing 0.2 to $20 \mathrm{ppm}$ benzene has been made and a GC procedure has been developed for analys is with a precision of better than one percent. The concentrations of these standard mixtures are currently being verified by comparison to benzene permeation tubes which have been calibrated gravimetrically. This will ultimately lead to a benzene in air Standard Reference Material (SRM) which has its accuracy derived from two independent bases. The procedure developed for benzene will serve as a prototype for the development of other high priority toxic organic gas standards.

Methods for preparation of accurate and stable standards of benzene and perchloroethylene in air have been studied. These particularly difficult gas systems have required the development of special techniques both for preparation of mixtures of predictable concentrations and for their subsequent analysis.

The increasing concern with the potential hazards associated with certain organic compounds in the ambient and industrial workplace atmospheres have empahsized the need to solve the difficult problem of standardization of the measurement of low concentrations of intermediate molecular weight volatile organic compounds. The compounds of interest include benzene, toluene, perchloroethylene, chloroform, carbon tetrachloride, ethylene dichloride, and tetrachloroethylene. Work completed several years ago at NBS indicated that mixtures of these compounds in air at high pressure in steel cylinders could not be prepared with predictable stability. However, aluminum cylinders, which have reduced adsorptive and absorptive properties of the inner surface compared to steel cylinders, appeared to be capable of containing organic mixtures with reduced losses.

Consequently, mixtures of benzene in air and perchloroethylene in air were prepared by a micro-gravimetric technique developed at NBS and the concentrations were determined over a period of several months. The 
benzene mixtures which ranged in concentration from 0.2 to $10 \mathrm{ppm}$ were found to have the same relative concentration, within the uncertainty of the analytical technique, over this period. The ususal difficulties associated with the analysis of sub-ppm concentrations of benzene in air samples were encountered during these determinations. The effects of the non-linearity of response to concentration ratios, the imprecision of integration for small peaks and the drift of the flame ionization detector response due to slow changes in instrument and sampling conditions were moderated by using multiple-standard calibration curves and careful sampling techniques. The elimination of the interference caused by a cylinder contaminant (probably residual trichloroethylene) was accomplished by the careful selection of the column parameter (i.e., length, material, temperature), and carrier-gas flow rates.

The gravimetric method used to prepare the air mixtures of benzene or perchloroethylene was devised to overcome the problem of transferring a vapor at low pressure into a cylinder without contamination by atmospheric air. The organic liquid was introduced into a previously weighed capillary tube which was then sealed and reweighed to determine the weight of the organic. The capillary tube was then placed in a short length of flexible tubing which in turn was connected to the weighed sample cylinder. The capillary was broken inside the flexible tube and the contents were flushed totally into the sample cylinder. The sample cylinder was then filled with the diluent gas whose mass was determined by difference after weighing the filled sample cylinder. Gravimetric standards with uncertainties in their concentrations of less than \pm 1 percent relative can be thus prepared.

Alternatively standards may be generated by permeation tubes containing the substance of interest. However, the accuracy of mixtures generated with permeation tubes depend to a great extent on the stability of the permeation rate. Tubes containing a number of different organic compounds were studied over a period exceeding one year. The results showed that tubes containing benzene required as much as one month to reach equilibrium at constant temperature. Tubes containing other organic compounds required greater or lesser times depending on the particular organic involved. The rates after equilibrium, however, were found to have uncertainties of less than \pm 1 percent relative.

It appears that permeation tubes may serve as adequate standards providing the conditions under which they are kept are carefully controlled and if frequent measurements of the permeation rate are made. 
(3) ACID PRECIPITATION QUALITY ASSURANCE SAMPLES

Participants: W. Koch, E. Garner

Background:

NBS had earlier prepared and analyzed three series of simulated rainwater reference solutions. The 1975 series consisted of four multicomponent solutions analyzed for metals, anions, conductivity, pH and acidity. In 1977, three multicomponent solution types (analyzed for all of the aforementioned) and one single component solution types (analyzed only for $\mathrm{pH}$, acidity and conductivity) were prepared. In 1979, two multicomponent and two single component solution types were prepared and analyzed. With the increase in interest in acid rain, the single component solutions (essentially dilute sulfuric acid) are becoming more important and are comming under closer scrutiny. At present, there is an insufficient data base to adequately address the problems at hand, most notably stability of these unbuffered solutions toward $\mathrm{pH}$ and acidity, and and an accurate protocol for the measurement of $\mathrm{pH}$ in unbuffered solutions. In addition, there is some concern that the existing solutions are too few in number and variety and that they do not cover the entire range expected in natural rainwater.

The basic components of the multi-year NBS research plan are:

1. Check the existing solutions for stability, especially $\mathrm{pH}$ and acidity over a two-year span.

2. Evaluate glass electrodes and reference electrodes for response time, stability, and accuracy in low conductivity unbuffered solutions. Develop a method for accurate and precise measurement of $\mathrm{pH}$ and acidity in such solutions.

3. Conduct research toward devising a stable chemical system having the following characteristics:
$0 \mathrm{pH} 3.0$ to 5.0
0 acidity $3 \mathrm{meq} / \mathrm{L}$ to $10 \mathrm{meq} / \mathrm{L}$
${ }^{0}$ conductivity 30 to $100 \mu \mathrm{S} / \mathrm{cm}$

Project Milestones

1. $F Y-1980$ :

0 Research and identify the problems and present limitations in the measurement of acidity and $\mathrm{pH}$ in rainwater. (Completed)

- Initiate a program to check the stability of existing reference solutions (Simulated Precipitation Reference Materials III) with regard to $\mathrm{pH}$ and acidity using potentiometric and coulometric techniques. (Completed) 
2. $F Y-1981$ :

${ }^{0}$ Continue stability check program.

- Evaluate various types and configurations of $\mathrm{pH}$ electrodes (glass and reference) in terms of response time, stability, and accuracy in low conductivity, unbuffered solutions.

${ }^{0}$ Evaluate the use of spectrophotometric methods for measuring $\mathrm{pH}$ in low conductivity, unbuffered solutions.

- Initiate research toward devising a stable chemical system having the following characteristics: $\mathrm{pH} 3.0$ to 5.0 ; acidity 3 to $10 \mathrm{meq} / \mathrm{L}$; conductivity 30 to $100 \mu \mathrm{S} / \mathrm{cm}$.

3. FY-1982:

- Conclude stability check program on Simulated Precipitation Reference Materials III.

- Continue research in a stable chemical system for acid rain reference material and produce a limited set of solutions for long-term stability testing.

${ }^{\circ}$ Develop protocol for $\mathrm{pH}$ and acidity measurements in rainwater.

\section{Project Progress-FY-1980}

1. Project Synopsis

A multi-year project concerned with acid rainfall was initiated. The scope of this project is to investigate and identify the problems and present limitations in the measurement of acidity and $\mathrm{pH}$ in rainwater. Based on the findings, research will be conducted to develop stable acid conductivity measurements in low ionic strength unbuffered solutions. This will require NBS competences in $\mathrm{pH}$, potentiometry, and conductimetry.

Work was initiated on checking the stability of existing reference solutions (Simulated Precipitation Reference rials I, II, and III) prepared by NBS for EPA audit programs. There appeared to be a stability problem with some of these solutions. In addition, a literature survey was initiated to identify problems and limitations in the measurement of acidity and $\mathrm{pH}$ in rainwater. This survey revealed serious deficiencies in acid rain measurements and the need for an adequate measuremient protoco1. A more detailed examination of the literature was undertaken.

Further investigation of stability on existing reference solutions contained in glass ampoules confirmed the presence of instability with some of these solutions. Further work is in progress to quantify the instability and its source. In addition, a small set of dilute sulfuric 
acid solutions (similar to Simulated Precipitation Reference Materials III, seried 940000 , but contained in C.P.E. bottles) was prepared. The stability of these solutions is being monitored with respect to $\mathrm{pH}$ and conductivity.

\section{Related NBS Activities}

During the past year, a programmable computing integrator was added to the ion-chromatograph which greatly expands its usefulness as an analytical tool. It is hoped that similar data aquisition and data management devices can be acquired for voltammetry, coulometry, and potentiometry to improve measurement capabilities and to handle routine mathematical operations.

In the coming year, in addition to an anticipated intense sampleanalysis effort, the Electroanalytical Chemistry Group will engage in research to develop a species-selective and sensitive anion detector for ion-chromatography based on amperometric principles. Investigations will be conducted as to the sources of and remedy for possible systematic errors in high-precision coulometric measurements which may adversely affect the accuracy of the method. 
Participants: W. E. May, W. J. Sonnefeld

Background:

Dilute solutions of polycyclic aromatic hydrocarbons (PAH's) in air or an inert gas matrix may be produced by adapting and modifying existing water generator column methodology. Studies undertaken to date seem to indicate that such gas solutions are saturated, and thus, thermodynamic constants can be determined. For example, the heat of vaporization of naphthalene was determined to be $16.5 \pm 0.1$ $\mathrm{kcal} / \mathrm{mole}$ using this method. This value compares favorably with the literature value of $15.6 \mathrm{kcal} / \mathrm{mole}$ ( $\mathrm{Zh}$. Fix Khim., 42,1814 (1968)). The gas solutions produceable by air generator columns are, by nature of being saturated at the generation temperature, standard PAH in air solutions, and may be utilized for measurement calibration purposes. However, the methodology required needs to be refined and application needs to be made to other PAH calibration compounds of higher ring size, including biologically important $\mathrm{PAH}$ such as benzo[a]pyrene. Initial work on this project will focus on anthracene, since reliable data exist for this $\mathrm{PAH}$ in the literature (API Monograph).

\section{Project Progress-FY-1980}

1. Project Synopsis

Work was initiated on this exploratory project to determine the feasibility of developing air generator columns as standards for generator columns as standards for generating accurate concentrations of PAH components in air. The technical approach was based on the evaluation of an air generator column containing a given PAH for measurement of $i$ ts vapor pressure as a function of column temperature by use of method, PAH effluent trapping, and HPLC analysis.

\section{Project Detail}

The feasibility of developing a Standard Reference Material for organic compounds in air is being investigated. A gas-saturation technique is being used to produce a saturated vapor solution of an organic compound at its thermodynamic equilibrium vapor pressure. The vapor is cryogenically trapped and then analyzed by coupled-column high-performance liquid chromatography.

The gas saturation method for the determination of vapor pressures dates back to 1845 . However, due to the lack of sufficiently sensitive analytical techniques, this method has not been extensively used until recently. Cliath and Spencer (Report to NAS-NRC Evaluation Panel, 141, (1978)), in a series of experiments, utilized the method to study desorption isotherms of pesticides. Pella (Anal. Chem., 48, 1632 (1976)), coupled a gas-saturator to a gas dilution system to produce a calibration system for exploring vapor detectors. May (Environ. Sci. Technol., $\underline{3}$, $670(1969))$, used the dynamic-coupled-column HPLC technique to determine the vapor pressure of naphthalene. Comparison with published data showed that a systematic error was present in this work. 
This study has been concerned with eliminating the systematic errors discovered earlier and extending the technique to vapor-phase studies of the larger polynuclear aromatic hydrocarbons. The dynamiccoupled-column HPLC system has been modified to include dual 6-port valves which allow for purging of the collection assembly by an inert gas. The techniques of column conditioning used for the water SRM generator columns have been applied to this work in order to properly remove any impurities from the starting material. The system is designed to permit continuous flow through all channels so as to eliminate sorption effects. Thus, the system is dynamic in nature. The generator columns and all connecting flow lines are constantly submerged in an isothermal bath. The vapor is concentrated on either an octadecyl silane-packed column (C-18) or via a cold trap assembly. The initial work has used an anthracene generator column. The concentration at $25^{\circ} \mathrm{C}$ has been determined as $60.2 \mathrm{ng} / \mathrm{L}$ with a precision of 1.4 percent relative.

Studies of the dependence of the flow rate upon the vapor density have shown that the vapor density remains constant for flow rates below $14 \mathrm{~cm}^{3} / \mathrm{min}$.

Initial temperature studies (Table 18) have shown excellent agreement with extrapolated data obtained by other techniques.

Future work will include studies of several columns containing varying concentrations of anthracene-coated $\mathrm{SiO}_{2}$. The effects due to the presence of other compounds and that due to the water vapor concentration will also be studied. Finally, a systematic check will also be used to determine if any systematic errors are present. 
Table 18. Preliminary Determination of the Polycyclic Aromatic Hydrocarbon Vapor Pressures as a Function of Temperature.

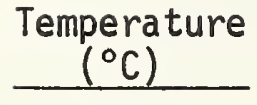

15.3

20.4

25.0

30.1

34.9

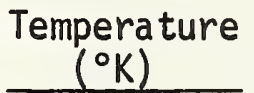

293.6

298.2

303.3

308.1
6.29

6.39

6.19

\section{Vapor \\ Pressure $\left(\right.$ torr $\left.\times 10^{6}\right)$}

2.68

2.57

2.75

4.51

4.71

4.59

11.4

11.6

11.1

20.3

19.9

19.4

As soon as the technique has been completely verified, it will be used to obtain the vapor pressure curves at ambient temperature for a series of polycyclic aromatic compounds. These data are presently available only by extrapolation of data obtained at higher temperatures.

Further applications will involve the use of the generator columns for calibration of high-volume atmospheric sampling systems. 
(5) OZONE PHOTOMETERS

Participants: A. Bass

\section{Background:}

The ozone layer in the stratosphere protects 211 forms of life at the surface of the earth from excessive exposure to solar ultraviolet radiation. There is great concern that the emission of substances such as nitrgoen oxides and halocarbons into the atmosphere may modify the ozone layer and cause possible harm to life on earth. Measurement of ozone concentrations in the upper atmosphere of the earth is essential in order to detect changes that may be caused by man's activities. Total ozone column density and vertical profiles are determined mainly by means of ultraviolet (UV) absorption spectrophotometry. A specially designed NBS research spectrometer had been designed for accurate ozone measurements. EPA, under its authority in the Clean Air Act, is required to assure accurate measurements of atmospheric ozone as a pollutant, as well to assess stratospheric ozone protection. EPA has requested that NBS design a standard ozone photometer based on NBS experience with the research instrument that, upon replication can be placed in EPA Regions for national ozone measurement quality assurance.

\section{Project Milestones and Estimated Completion Dates}

1. FY-1980:

- Develop instrument performance specifications relative to accuracy and precision. (Completed)

$5 / 80$

- Initiate prototype instrument design and component testing. (Initiated)

$6 / 80$

2. FY-1981:

- Design of prototype instrument and test components of the prototype in combination with one another.

- Complete preliminary prototype instrument design.

- Fabricate prototype photometer.

$9 / 81$

3. FY-1982:

- Performance testing and verification of prototype photometer relative to design specifications (EMSL/RTP and NBS).

- Complete final design of photometer and develop revised cost estimate, if any.

- Fabricate, test, and deliver three photmeters to EPA. 6/82 


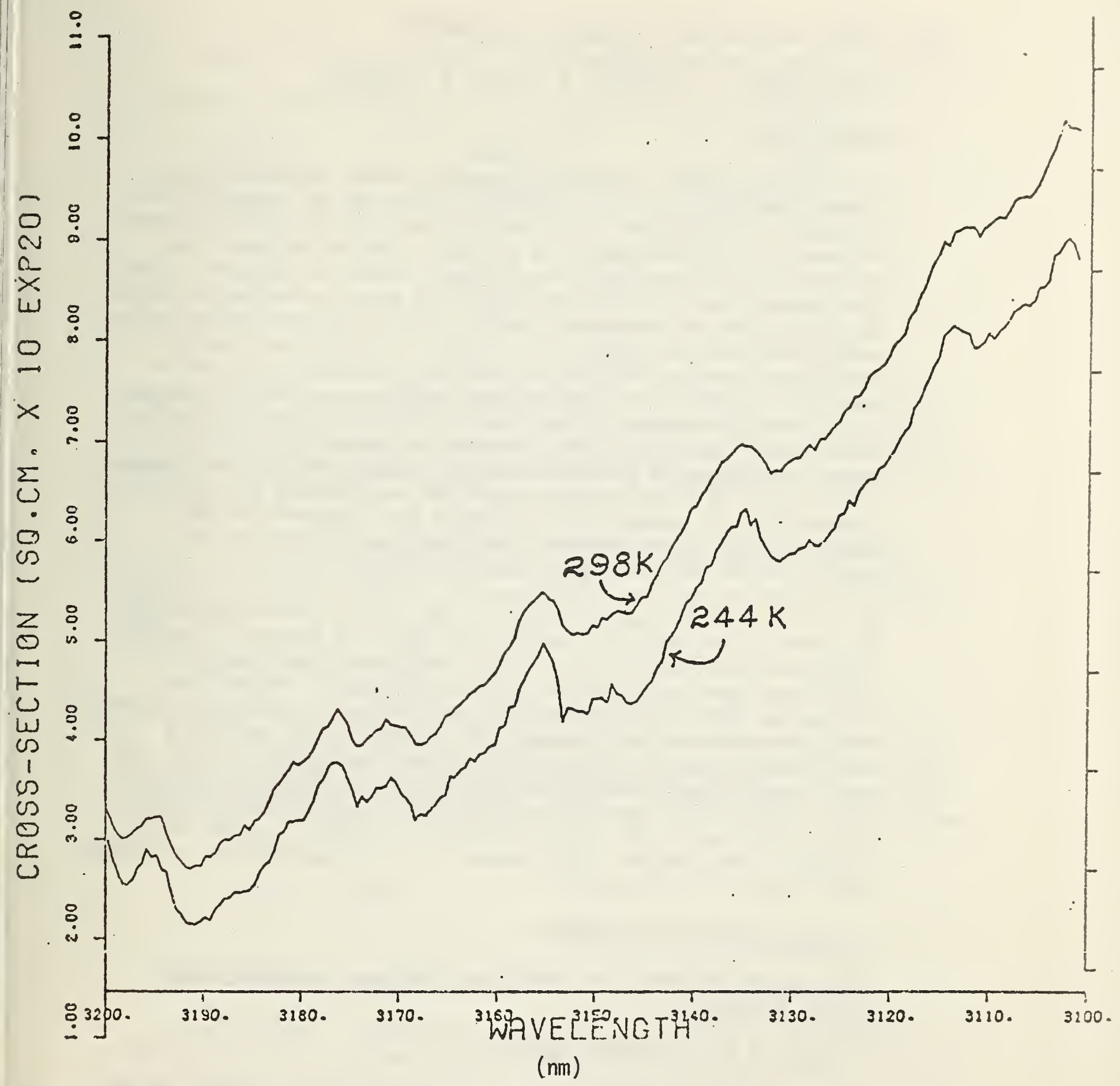

Figure 14. Absorption spectrum of ozone at $298 \mathrm{~K}$ and $244 \mathrm{~K}$. Spectral slit width $0.02 \mathrm{~nm}$; wavelength scanned in steps of $0.05 \mathrm{~nm}$. 
Participants: E. Hughes, W. Dorko, G. Sleater

Background

Due to the Nation's need for increased energy production, the abundance of major natural resources that may be used for this purpose in the Western U.S., and the anticipated marked increase in power plant development in the West, EPA established the Western Air Quality Monitoring Project under its Western Energy/ Environment Monitoring Study (WEEMS) to provide monitoring data to assess the environmental impact produced by present/projected energy-related development activities. In addition, the conversion of power plants to coal-firing is on the increase in the U.S. In order to provide data quality assurance in quantitating energy-related air pollutant emissions associated with conventional and fossil fuel conversion technologies, EPA recognized the need for the development and provision of gaseous SRM's by NBS and the need to establish a mechanism to insure traceability of commercially-prepared gas standards to NBS SRM's for quality assurance in national activities for the monitoring of pollutant gas concentrations in air.

As in the past the major output of the NBS Gas Metrology Group has been in the analysis and certification of gaseous Standard Reference Materials (SRM's). Over 3400 individual samples of SRM's have been analyzed during the past year. In addition, 85 gaseous SRM's sold by the Office of Standard Reference Materials have been returned by the users for reanalysis as part of a continuing study of the stability of gas standards. It is anticipated that seventeen new SRM's will be issued by the end of FY 1981. These new SRM's include low concentrations of sulfur dioxide in air, nitric oxide in nitrogen, oxygen in nitrogen, and propane in nitrogen, all at concentrations extending the range of existing SRM's.

\section{Project Progress - FY-1980}

1. Development of SRM's for Stationary Sources Associated with Energy Production

(a) $\mathrm{NO}_{2}$ in Air Gas Blend SRM's

The four levels of gas mixtures, 250, 500, 1000 and $2500 \mathrm{ppm} \mathrm{NO}$ in air, were received and each batch was evaluated to determine homogeneity. The batches at 250 and $2500 \mathrm{ppm}$ were produced by one supplier and the batches at 500 and 1000 ppm were provided by another supplier. 
The results of the first set of intercomparisons are given as follows:

Intercomparisons of $\mathrm{NO}_{2}$ in Air Cylinders for Certification

Nominal

Concentration

(ppm)

Range of

Concentration

250

250
500

1000

(ppm)

s.d. of the

Concentration

2500

17

18

12

45

(ppm)

$$
\begin{array}{r}
5 \\
5 \\
3 \\
12
\end{array}
$$

The lesser standard deviation relative to the range of values reflects the fact that most samples had concentrations close to the average but that in each lot there were several outliers. A second analysis of all samples is essential to determine whether the outliers represent unstable samples or samples whose concentration fell at a lesser value at the time of preparation.

The results were encouraging, however, since a larger spread in the value was expected. A second analysis of the four sets was conducted in April 1980. The four SRM's are planned for completion by March 1981.

\section{(b) $\underline{\mathrm{SO}}_{2}$ in $\mathrm{N}_{2} \mathrm{SRM}^{\prime} \mathrm{s}$}

Orders were prepared for the purchase of 50 and $90 \mathrm{ppm} \mathrm{SO} \mathrm{S}_{2}$ in $\mathrm{N}_{2}$. The laboratory standards necessary for the certification of the 50 and $90 \mathrm{ppm} \mathrm{SO}{ }_{2} \mathrm{SRM}^{\prime} \mathrm{s}$ were prepared and evaluated. Both a chemical method (hydrogen peroxide method) and gas chromatography will be used in the certification. The SRM's are planned for completion by December 1980 .

\section{(c) NO in $\mathrm{N}_{2}$ SRM's $^{2}$}

SRM's have been issued for 1500 and $3000 \mathrm{ppm}$ NO in $\mathrm{N}_{2}$.

(d) $\underline{0}_{2}$ in $\mathrm{N}_{2}$ SRM's

Two new SRM's (SRM \#2657 and \#2658) consisting of two and ten mole percent $\mathrm{O}_{2}$ in $\mathrm{N}_{2}$ were completed.

(e) $\mathrm{CO}_{2}$ in Air SRM's

Development of standards for the accurate measurement of carbon dioxide have progressed to where excellent agreement with accepted international standards have been achieved. As a result, a large number of samples are being prepared commercially which will be used to study the feasibility of large scale production of highly accurate and stable $\mathrm{CO}_{2}$ gas standards. If production on a large scale is feasible then these standards, as SRM's, will be integrated into the existing measurement network for atmospheric carbon dioxide. 


\section{Automation of Gas Analysis}

A second Computer-Operated Gas Analysis System (COGAS-II) has been constructed, and is in use for certification analysis of SRM's. A third system (COGAS-III) has been constructed for the automation of permeation tube calibrations. Preliminary results indicate that the system will operate as intended.

Under a separate program with the Motor Vehicle Manufactures Association, ten new carbon monoxide in nitrogen mixtures were developed involving measurements which demonstrated the usefulness of high-pressure injection from large sample loops into a gas chromatograph to increase detector response for carbon monoxide mixtures at concentrations of less than $100 \mathrm{ppm}$. The technique allowed the intercomparison of samples with standards with a precision of \pm 0.2 percent. Certification of these SRM's involved the first test of the software used to control the combined gas chromatography NBS-developed computer operated-gas analys is system (COGAS). COGAS had previously been used only with continuous flow gas analyzers.

\section{Development of an NBS/EPA Traceability Procedure for Gas Standards}

Preliminary reviews of a procedure for establishing traceability of commercially prepared mixtures to certain gaseous SRM's were completed. A meeting with representatives of the gas industry was held to discuss the practicability of the NBS developed procedure relative to the stateof-the-art both of the preparatory processes and the analytical procedures.

The demand for gas standards is increasing at an accelerated rate. Improvement in instrumentation during the last two decades and mandated requirements for accuracy in gas measurements related to environmental concern have both contributed to the demand. Limitations imposed on the gaseous SRM program resulted in severe shortages of standards which on occasion has caused concern among regulatory agencies, users and producers. Since it is not realistic, either philosophically or physically, to consider expanding the gaseous SRM program beyond its present bounds, it has become essential to establish some alternative supply of accurate standards. As a result of this need a protocol has been developed which in essence transfers the responsibility for producing large numbers of accurate standards (Certified Reference Materials) to the specialty gas industry. The protocol makes provision for monitoring by the regulatory agencies and at the same time NBS retains the responsibility for assuring traceability of the Certified Reference Materials (CRM's) to NBS gaseous SRM's.

According to the protocol, the specialty gas producer will prepare CRM's at concentrations equal to or very close to the concentration of existing SRM's. These will be prepared in large homogeneous batches. The producer will analyze each CRM from the batch by comparison to an SRM. The regulatory agency will select samples from each batch for analysis. The analysis by the regulatory agency will be performed using 
SRM's as calibrants. Both the producer and the regulatory agency will furnish NBS with complete information concerning the production and analysis of the CRM's including the data for both calibration of the instruments used and the analysis of the CRM's. NBS will then statistically compare the results reported by the producer with those found by analysis by the regulatory agency. In addition, NBS will examine all the results reported by the producer to ascertain the homogeneity of the batch. From these data it will be possible to verify the total uncertainty of the concentration claimed by the producer. At this point the concerned parties will be notified that the particular batch of CRM's has been prepared in accordance with the protocol and that the stated concentration is correct within the defined limits of uncertainty.

The protocol in its present form has been extensively modified in response to comments from the gas industry, users of SRM's and the principal regulatory agency, the Environmental Protection Agency. It is not a "cook book" by which CRM's can be prepared. Rather, it recognizes the experience and expertise of the specialty gas industry while at the same time it recognizes the reservations which the user may have regarding the industry and which the industry may have regarding the regulatory agency. It is expected that the role of NBS as an independent overseer will inspire confidence not only in the quality of the CRM's but in the integrity of the entire procedure.

The availability of CRM's should moderate the demand for the corresponding SRM's, so that the latter would be used largely as primary standards, which was the purpose for which they were originally produced. A document has been prepared for publication under the Interagency Energy-Environment Program, entitled "A Procedure for Establishing Traceability of Gas Mixtures to Certain National Bureau of Standards Standard Reference Materials." 


\section{(7) ENERGY-RELATED PARTICULATE STANDARDS DEVELOPMENT}

Participants: P.A. Pella, W. Koch, E.C. Kuehner, J. Sieber, C. C. Gravatt

\section{Background:}

In concert with the needs to develop energy-related gas standards (q.v.), development of particulate standards is needed to provide data quality assurance in the monitoring of particulate emissions. This need is magnified by the anticipated increase in conventional fossil fuel combustion and the anticipated increased activities in converted fossil fules and oil shale to address the Nation's increasing energy needs. EPA has placed an empahsis on both total and fine particulate emissions monitoring.

The activities in the EPA/NBS E/E program for FY 1980 for particulate standards development have covered: (a) the evaluation of standards for x-ray fluorescence analysis; (b) development of $\mathrm{Pb}$ (in addition to sulfate and nitrate) on glass fiber filter standards; and $(c)$ work on the air particulate collection in the Washington, DC area.

\section{Project Progress-FY-1980}

1. Development of Thin Glass Films As Potential Standards for X-ray Spectrometric Calibration

$X$-ray fluorescence spectrometry has proven to be a versatile and rapid method for multielement analysis in many analytical applications. of particular interest are substances which can be analyzed in the form of a deposit on a filter, mesh or membrane, or as a thin pressed pellet. collected airborne particulate matter, particulates in waste water and geochemical samples are just a few examples of this type. In some respects such "thin samples" are ideal for x-ray fluorescence analysis because the detection limits are suitably low and interelement effects such as $x$-ray absorption and/or enhancement are negligible. The absence of any sizable interelement effects makes possible a linear instrument response to element mass per unit area. Spectrometer calibration for analysis of such samples is usually performed with thin samples containing known concentrations of the analyte(s) of interest. Some problems associated with the "thin specimen model" are $x$-ray self-absorption in particles (notably for the light elements such as Al, Si, P, and S) commonly referred to as particle-size effects, and self-absorption in the substrate. Both of these effects require the application of correction factors. Therefore, the basic premise is that accurate multielement analysis of thin samples can be performed based on the thin specimen model, together with empirical or theoretical correction factors. 
There are several important criteria which need to be met if thin samples are to be acceptable for calibration purposes. First, the uniformity of the sample as well as its homogeneity must be within an acceptable well-defined tolerance limit. If the sample contains particulate matter, both the size and distribution should be characterized. Secondly, the mass loading of the sample must be known to an acceptable degree of accuracy. And finally, the sample should possess sufficient durability and integrity.

The goal of a joint program between NBS and the U.S. Environmental Protection Agency (EPA) was to perform the research and development required to produce thin standard samples especially useful for calibration of x-ray fluorescence analysis of collected airborne particulate matter. For this purpose we have investigated several candidate methods for preparing such samples.

One method which shows great promise is the deposition of thin films of glass containing known concentrations of several elements of interest on polycarbonate substrates by a technique known as focused ion-beam sputtering. Ion-sputtering essentially involves the transfer of atoms and/or molecules from a target to a substrate by the action of an ion beam (usually argon) incident upon the target. The ion beam is accelerated at high potential (700-1100 volts) with a flux of about 0.5 $\mathrm{mA} / \mathrm{cm}^{2}$ toward the target at a $45^{\circ}$ angle. Since the substrate is not in the plasma environment, substrate damage does not occur. This is a distinct advantage of this particular sputtering technique. Other advantages to be gained are: (1) production of thin films from synthetic materials such as glasses of known elemental composition which can be varied as desired; (2) high adherence of the glass to the substrate which obviates the need for overcoating to maintain sample integrity; and (3) easy control of the glass mass loading on the substrate by variation of the sputtering time.

Several studies were performed with glass targets fabricated at NBS which allowed up to eight $47 \mathrm{~mm}$ substrates to be coated at one time. Instrumental parameters such as ion accleration voltage and ion current were systematically varied to determine their effect on film composition. It was found that rather severe changes in these parameters of ten do not affect the film composition by more than \pm 15 percent. Glass films sputtered from targets containing phosphorous and sulfur were also prepared to demonstrate the feasibility of preparing films containing elements of low atomic number.

In Table 19 are summarized the elemental compositions of three sets of films at mass loadings ranging from 167 to $185 \mu \mathrm{g} / \mathrm{cm}^{2}$ which were prepared at various times. These data demonstrate that the compositional reproducibility of thin films from set to set is satisfactory for the purposes of preparing standard films. 
Multi-element thin standards suitable for calibration purposes can be fabricated by the focused ion-beam sputtering technique. In order to include all the elements of interest in any particular film, several glass targets would need to be fabricated. It is believed that these films would be useful to monitoring agencies such as EPA for assessing environmental impact of airborne particulate matter. Work is now in progress to prepare several sets of films which will be certified by the Office of Standard Reference Materials as NBS Standard Reference Materials.

\section{Particulates on Glass Fiber Filters: Certification of} SRM's for $\mathrm{Pb}$, Sulfate, and Nitrate on Filter Media

Final certification of SRM 2673, Sulfate and Nitrate on Filter Media, and SRM 2674, Lead on Filter Media, has been completed. These materials are intended for use in the evaluation of apparatus and methods used in the determination of lead, sulfate, or nitrate in atmospheric particulates collected on filters.

Certification measurements for the anions were accomplished by ion chromatography. Lead for SRM 2674 was determined by both voltammetry and atomic absorption spectrometry (AAS). Statistical analysis of the data indicated no significant effects of biases in the AAS due to undissolved solids from the glass filter matrix.

Copies of the certificates of analysis are included for information.

\section{Urban Air Particulate, Washington, D. C. Sample}

In August 1979, the collection of the Washington, DC, urban particulate sample was terminated. The bags were vacuumed out using the same system as that described for the St. Louis material, and approximately 45 pounds of unsieved material was obtained. Several sieving strategies were evaluated in order to develop one that was effective in removing bag fibers, but did not excessively reduce the non-bag-fiber fraction of the sample. It appears that 30-35 $\mathrm{lb}$. of sieved material will be available for final analysis. Preliminary inorganic, organic, and particle size analysis has been performed on the Washington material. The analysis indicated that the Washington material can be used either as a replacement for the St. Louis material or as an organic certified sample. 


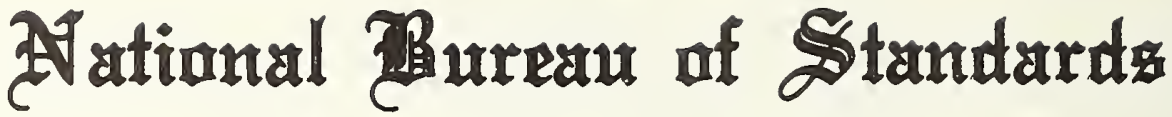

\section{Cilertifitate of Analygis}

\section{Standard Reference Material 2673}

\section{Sulfate and Nitrate on Filter Media}

This Standard Reference Material is intended for use in the evaluation of apparatus and methods used in the determination of atmospheric particulate sulfate and nitrate which have been collected on filters. It consists of a series of filter strips upon which sulfate and nitrate have been deposited in an essentially central location. The values certified correspond to the quantities of the substances leached from the filter strip without destruction of the filter matrix.

\begin{tabular}{|c|cc|cc|}
\hline \multirow{2}{*}{$\begin{array}{c}\text { Sample } \\
\text { Number }\end{array}$} & \multicolumn{2}{|c|}{ Sulfate content $\mu$ g/filter } & \multicolumn{2}{c|}{ Nitrate content $\mu \mathrm{g} /$ filter } \\
\cline { 2 - 5 } & Average value & Tolerance limits & Average value & Tolerance limits \\
\hline \multirow{2}{*}{ I } & 503 & $493-513$ & 100 & $98-102$ \\
II & 2002 & $1955-2049$ & 1002 & $978-1026$ \\
III & 6939 & $6635-7243$ & 2513 & $2404-2622$ \\
Blank & 2 & $0-6^{*}$ & 2 & $0-3^{*}$ \\
\hline
\end{tabular}

- Range of measured values

The average value is the mean based on the analysis of 24 or 28 filters randomly selected from the lot. The tolerance limits are determined so that at the $95 \%$ confidence level they will contain the central $95 \%$ of the population of filter values. Details of the preparation, analysis, and statistical treatment of the data are given on the reverse side of the certificate.

This Standard Reference Material was prepared by B. I. Diamondstone. The analytical measurements were made by W. F. Koch. Statistical analysis of the data was provided by J. Orban. The overall direction and coordination of the preparation and analytical measurements leading to certification were performed in the Center for Analytical Chemistry under the chairmanship of J. K. Taylor.

The technical and support aspects involved in the preparation, certification, and issuance of this SRM were coordinated through the Office of Standard Reference Materials by W. P. Reed.

Washington, D.C. 20234

June 25, 1979
George A. Uriano, Chief

Office of Standard Reference Materiak 


\section{Preparation}

This SRM consists of strips cut from glass fiber filters, such as are commonly used for measurements of atmospheric particulates, using high volume samplers. Solutions containing known amounts of potassium sulfate and potassium nitrate were prepared, gravimetrically, and multiple aliquots of $25 \mu \mathrm{L}$ were placed on the filter strips. Four aliquots were so transferred to each filter in the case of samples I and II, and five in the case of Sample III. The pipets were calibrated by weighing similar aliquots transferred into weighing bottles.

The filters were prepared in a clean room and allowed to air-dry before packaging in glassine envelopes. The filters were prepared in groups of 100 and the proper number of aliquots were dispensed into weighing bottles at the beginning and end of each sequence, to monitor the performance of the pipet.

\section{Analytical Measurements}

Twenty four samples were randomly selected from the production lot (28 in the case of sample III) for chemical measurement of their extractable sulfate and nitrate. Each filter strip was extracted with a standard eluent (see below) for 15 minutes in a $55^{\circ} \mathrm{C}$ ultrasonic bath. The extracts were analyzed for sulfate and nitrate by ion chromatography. The eluent was a solution of $0.003 \mathrm{M} \mathrm{NaHCO}_{3}$ and $0.0018 \mathrm{M} \mathrm{Na}_{2} \mathrm{CO}_{3}$. A $100 \mu \mathrm{L}$ sample loop was used. Peak heights were compared with those obtained from accurately prepared standard solutions. Three standards were prepared for each concentration level so as to bracket the extracts.

The randomly selected filters were also analyzed in random order. No significant systematic errors were observed related to the order of preparation. Average values and the overall standard deviation, $s_{0}$, of individual measurements were calculated. This standard deviation includes measurement error and filter content error.

The standard deviation, $\mathrm{s}_{\mathrm{f}}$, due to filter content variability was computed from a sample of 12 or 14 weighed quantities delivered by the pipets during the preparation of the filters. The resulting tolerance interval for the sulfate/nitrate contents is of the form

$$
\overline{\mathrm{X}} \pm \mathrm{ksf} \text {. }
$$

It is this tolerance interval that should be of greater interest to the participating laboratory since it gives practical bounds for the likely values of the sulfate or nitrate contents that might be found in a given filter.

The following table lists the values of $\mathrm{s}_{0}$ and $\mathrm{s}_{\mathrm{f}}$ for each set of filters. The measurement standard deviation, $\mathrm{s}_{\mathrm{m}}$, for NBS can be computed using the relation $\mathrm{s}_{0}^{2}=\mathrm{s}_{\mathrm{m}}{ }^{2}+\mathrm{s}_{\mathrm{f}}{ }^{2}$

\begin{tabular}{rrrr}
\multicolumn{1}{c}{ Set } & Average $(\mu \mathrm{g})$ & \multicolumn{1}{c}{ so } & \multicolumn{1}{c}{ sf } \\
\hline I $\mathrm{SO}_{4}$ & 502.6 & 8.89 & 3.14 \\
$\mathrm{NO}_{3}$ & 100.3 & 2.56 & .63 \\
$\mathrm{II} \mathrm{SO}$ & 2001.7 & 29.30 & 14.88 \\
$\mathrm{NO}_{3}$ & 1001.7 & 15.92 & 7.44 \\
III SO & 6939.0 & 109.35 & 100.75 \\
$\mathrm{NO}_{3}$ & 2513.0 & 43.70 & 36.30
\end{tabular}

The average values calculated from the composition of the solutions and the quantitites delivered by the pipets are in general agreement with the analytical values. However, the values certified are those obtained by analysis.

\section{Recommended Usage}

The material is not homogeneously distributed on the filter; hence the sample must be used in its entirety for analysis. It is recommended that the filter be extracted at $55^{\circ} \mathrm{C}$ in an ultrasonic bath, with water or other nonreactive solvents. The filter base should not be digested to put it into solution. 


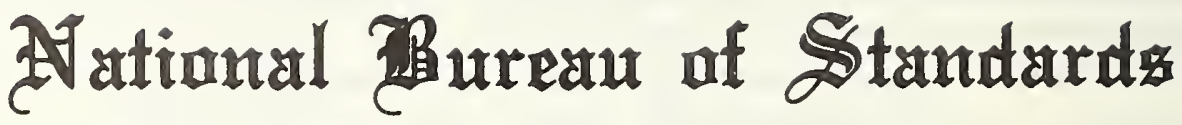 UTertifirate af Anallyo}

\section{Standard Reference Material 2674}

\section{Lead on Filter Media}

This Standard Reference Material is intended for use in the calibration of apparatus and the evaluation of methods used in the determination of atmospheric particulate lead which has been collected on filters. It consists of a series of filter strips upon which lead has been deposited in an essentially central location. The values certified correspond to the quantities of the substance leached from the filter strip without destruction of the filter matrix.

\begin{tabular}{|c|c|c|}
\hline \multirow{2}{*}{ Sample No. } & \multicolumn{2}{|c|}{ Lead Content, $\mu \mathrm{g} /$ filter } \\
\cline { 2 - 3 } & Average Value & Tolerance Limits \\
\hline I & 100 & $97-103$ \\
II & 303 & $294-312$ \\
III & 1505 & $1477-1533$ \\
Blank & 1.4 & $0.7-2.1^{*}$ \\
\hline
\end{tabular}

*Range of measured values

The average value is the mean based on the analysis of 26 or 28 filters randomly selected from the lot. The tolerance limits are determined so that at the $95 \%$ confidence level they will contain the central $95 \%$ of the population of filter values. Details of the preparation, analysis, and statistical treatment of the data are given on the reverse side of this certificate.

This Standard Reference Material was prepared by B. I. Diamondstone. The analytical measurements were made by E. J. Maienthal and M. S. Epstein of the Inorganic Analytical Research Division. Statistical analysis of the data was provided by J. Orban of the Statistical Engineering Division.

The overall direction and coordination of the preparation and analytical measurements leading to certification were performed in the Center for Analytical Chemistry under the charimanship of J. K. Taylor.

The technical and support aspects involved in the preparation, certification, and issuance of this SRM were coordinated through the Office of Standard Reference Materials by T. E. Gills.

Washington, D.C. 20234

December 18, 1979
George A. Uriano, Chief Office of Standard Reference Materials 


\section{Preparation}

This SRM consists of strips cut from glass fiber filters of the type normally used in high volume samplers for the measurement of atmospheric particulates. Solutions containing known amounts of lead nitrate were prepared gravimetrically, and aliquots of $25 \mu \mathrm{L}$ were placed on the filter strips using micropipets. Four aliquots were transferred to each filter in the case of samples I and II and five in the case of sample III. The pipets were calibrated by weighing similar aliquots transferred into weighing bottles.

The filters were prepared in a clean room and allowed to air-dry before packaging into glassine envelopes. The filters were prepared in groups of 100 and the proper number of aliquots were dispensed into weighing bottles at the beginning and end of each sequence, to monitor the performance of the pipet.

\section{Analytical Measurements}

Twenty-eight filters were randomly selected from the production lot (26 in the case of Sample I) for chemical measurement of extractable lead. Selected filters were extracted with dilute nitric acid, using ultrasonic vibration to assist in the extraction. After the nitric acid extraction, the filters were thoroughly rinsed with distilled water to ensure complete extraction. The extracts were quantitatively diluted and analyzed by linear sweep voltammetry which had been calibrated with solution standards prepared from high purity lead.

The selected filters were analyzed in random order. No significant systematic errors were observed related to order of preparation. Average values were calculated together with the overall standard deviation, $s_{0}$, of individual measurements. This standard deviation includes measurement error and filter content error. The resulting confidence interval for the mean is of the form

$$
\overline{\mathrm{X}} \pm \mathrm{t} \mathrm{s}_{\mathrm{o}} / \sqrt{\mathrm{n}}
$$

where $s_{o}$ is based on $n-1=27$ degrees of freedom.

A selected number of filters were also analyzed by atomic absorption. The results obtained were well within the tolerance limits given and were consistent with the certified values.

The standard deviation, $\mathbf{s}_{\mathrm{f}}$, due to filter content variability was computed from a sample of 12 or 14 weighed quantities delivered by the pipets during the preparation of the filters. The resulting tolerance interval for the lead content is of the form

$$
\overline{\mathrm{X}} \pm \mathrm{ksf} \text {. }
$$

It is the tolerance interval that should be of greater interest to the participating laboratory since it gives practical bounds for the likely values of the lead content that might be found in a given filter.

The following table lists the values of $s_{o}$ and $s_{f}$ for each set of filters. The measurement standard deviation, $s_{m}$, for NBS can be computed using the relation $\mathrm{s}_{\mathrm{o}}{ }^{2}=\mathrm{s}_{\mathrm{m}}{ }^{2}+\mathrm{s}_{\mathrm{f}}{ }^{2}$

\begin{tabular}{lrrr} 
Set & Average $(\mu \mathrm{g})$ & \multicolumn{1}{c}{$\mathrm{s}_{\mathrm{o}}$} & \multicolumn{1}{c}{$\mathrm{s}_{\mathrm{f}}$} \\
\hline I & 99.7 & 1.59 & 0.89 \\
II & 302.9 & 5.37 & 3.00 \\
III & 1505.2 & 12.73 & 9.24
\end{tabular}

The average values calculated from the composition of the solutions and the quantities delivered by the pipets are in general agreement with the analytical values. However, the values certified are those obtained by the analysis.

\section{Recommended Usage}

The material is not homogeneously distributed on the filter, hence the sample must be used in its entirety for analysis. It is recommended that the filter be extracted at $45-50^{\circ} \mathrm{C}$ in an ultrasonic bath, with dilute nitric acid ( 2 $\mathrm{mL} \mathrm{HNO}_{3}+15 \mathrm{~mL} \mathrm{H} \mathrm{O}$ ). After extraction the filters should be thoroughly rinsed with distilled water. The filter base should not be dissolved. 


\section{AIR MEASUREMENTS - METHODS DEVELOPMENT}

a. Overview

New and improved methods of analysis are necessary if advancements are to be made in measurement technology for the characterization and assessment of air pollutants. Existing methods do not always adequately address characterization and quantification of pollutants derived from diverse sources or adequately measure personal exposure of toxic materials. The matrix of pollutants of national interest has become substantially more complex, due to growing environmental concerns in such areas as toxic substances and developing energy technologies (coal conversion, shale 0i1). Hence, method development activities at NBS must focus not only on the development or improvement of methodology for certification of needed national standards (research materials and SRM's), but must attend the present and intermediate range needs for advanced measurement methods and instrumentation that insure a viable continuity in the development of measurement capabilities for air pollution measurements. In addition to the qualitative and quantitative aspects of new or improved measurement techniques, measurement uncertainties must be well characterized in order to insure the reliability of conclusions based upon the experimental data derived from field measurements.

\section{Objectives}

To develop new or improved methodologies and advanced instrumentation or devices that provide or enhance data quality assurance in national air quality evaluations related to the characterization and monitoring of air pollutants (e.g., from industrial and energy-producing sectors, automobile and jet-airplane emissions). The developed methodologies and instrumentation will serve to assist the establishment of national criteria in air pollution abatement and air quality improvement, and will provide relevant technology for reference methods, reliable field measurement calibrations, and the development of RM's and SRM's as national standards.

\section{Interactions}

This Task strongly interacts with various NBS centers (e.g., Center for Analytical Chemistry, Center for Thermodynamics and Molecular Science, Center for Radiation Research, Center for Applied Mathematics) and NBS Offices (OSRM, OSRD). Initiation, performance, and modifications to the activities of the prograni involve direct interactions between the involved technical and managerial staff of NBS and relevant staff complements within the U.S. Environmental Protection Agency, or with other governnient agencies unde" Interagency Agreements or Other Agency contracts. Strong interactions also exist with industry, State and local government agencies, research laboratories, and universities. Related NBS activities in the inprovement or development of methods, devices, or instruments having application to the measurement of air pollutants are close?y followed. 


\section{Task Justification}

Development of inproved methodologies and technologles for data quality assurance is essential for valid evaluations of air quality in areas of concerns for the protection of public heal th and the national ecology. Attainnent of these national goals is hence directly tied to the generation of reliable environmental measurements. The activities of this Task are required, provided under, or have been recommended by the Clean Air Act and its 1977 amendments, the Standing Air Monitoring Working Group, the Couricil on Enviornmental Quality, the Interagency Task Group on Environmental Data and Monitoring, the Toxic Substances Control Act, and the NBS Organic Act. This Task is designed to address national priority needs for measurement methods for air data quality assurance.

b. List of Task Projects

Project Title

Personal Monitors

UV Spectroradiometry

Gases

Particulates

Environmental Assessment
Source of Funding*

$$
\begin{aligned}
& P / T \text { - EPA } \\
& P / T \text { - EPA } \\
& E / E \text { - EPA } \\
& E / E \text { - EPA } \\
& B E P \star \star
\end{aligned}
$$

\footnotetext{
*P/T: Pass Through - E/E: Energy/Environment

* *Bureau of Engraving and Printing

Dept. of the Treasury
} 


\section{(1) PERSONAL EXPOSURE MONITORS}

Participants: Jimmie Hodgeson, Barry Cadoff, David Bright, Robert Fletcher, Alex Fatiadi

\section{Introduction}

Regulatory decisions on air pollution control which involve direct and indirect costs of billions of dollars, are often made without adequate knowledge of the health impacts of air pollution. The weakest link in health effects studies is our knowledge of individual exposures. The importance of population exposure estimates in air pollution health effects studies makes it imperative that future studies include exposure estimates more representative of what people breathe. Studies of air pollution health effects have usually relied on one or several fixed monitoring stations to provide data for an estimate of the exposure received by an entire neighborhood. Epidemiologists have begun to call for something better. Several recent meetings of specialists in the field of air pollution health effects have led to recommendations urging the prompt development of small, portable individual air pollution monitors.

The overall goal of the NBS program will be to develop principles, concepts, and actual devices for determining personal exposure to critical air pollutants. Both passive and active monitors will be developed to provide long-term integrated exposures and short term events. This narrative addresses the first year of the program, and the pollutants EPA has identified as having the highest priority - fine particulates, nitrogen dioxide, toxic organics, and ozone.

\section{Nitrogen Dioxide}

Our first approach will be to investigate passive samplers for $\mathrm{NO}_{2}$. Edward Palmes of New York University Medical Center presented a paper at a recent symposium in which he demonstrated indoor measurements of ambient levels of $\mathrm{NO}_{2}$ with a passive sampler. He used a simple diffusion tube, $7 \mathrm{~cm}$ in length and $1 \mathrm{~cm}$ in diameter. Forty by 40 mesh stainless steel screens coated with triethanolamine (TEA) are placed in the bottom of the tubes. TEA is a standard reagent for collection of $\mathrm{NO}_{2}$ as nitrite in solution. After exposure the nitrite is extracted and analyzed colorimetrically using standard GriessSaltzman reagent. Palmes used this approach to obtain indoor exposure data and measured average values ranging from 50 to $200 \mathrm{ppb}$. He has not determined sensitivity, accuracy and specificity, nor has he compared his results with continuous measurement of $\mathrm{NO}_{2}$. 
We propose as the first step, an evaluation of Palmes procedure for the parameters mentioned above using an exposure chamber which is monitored with a chemiluminescence $\mathrm{NO}_{x}$ analyzer. In addition, we propose to compare the diffusion tube with ${ }^{x}$ a larger area passive sampler consisting of a permeable membrane backed by the collecting substrate. TEA has been shown to have a poor collection efficiency for $\mathrm{NO}_{2}$ in solution (ca. 50\%). Investigators at EPA-RTP have shown that the addition of 0methoxy-phenol (guaiacol) increases the collection efficiency and this will be investigated. Finally, we propose to evaluate ion chromatography as the method for analysis of nitrite or nitrate (after oxidation in solution).

EPA has expressed an interest in continuous or semi-continuous personal monitors to obtain records of maximum hourly exposures. During the first year we propose to provide a research plan for the development of such a device. The most promising approach at present appears to be a voltammetric sensor which is similar in principle to the commerically available Ecolyzer personal monitor for $\mathrm{CO}$. In the case of $\mathrm{NO}_{2}$, the major problem to be overcome with this type of sensor will be interferences. Several common air pollutants are active at the redox potentials required for $\mathrm{NO}_{2}$. Thus we plan to research the feasibility of miniaturizing other Eypes of continuous monitors for $\mathrm{NO}_{2}$.

Some work has al ready been carried out regarding the development of an analytical procedure for a passive personal monitor for $0_{3}$. Unlike $\mathrm{NO}_{2}$, an acceptable analytical work-up does not exist for collected $\mathrm{O}_{3}$. A preliminary evaluation has been made of a promising fluorometric technique. Collected $\mathrm{O}_{3}$ reacts with non-fluorescent 9,10-dihydroacridine or 9,10-dihyroanthracene to form intensely fluorescent acridine or anthraquinone. The intensity of the fluorescene produced is a linear function for very low concentrations of $0_{3}$. The inherent sensitivity of this fluoremetric approach should provide a passive technique wi th short time resolution. Any further work on the ozone techniques will be aeferred, with emphasis placed on $\mathrm{NO}_{2}$.

\section{Fine Particulates}

Very little work has been done on personal exposure devices for fine particulates at ambient levels. We propose a dual and simultaneous approach. In one approach, research will be devoted to development of an active device consisting of a mininature filter, with a coarse and fine particle separator coupled to a miniature pump. The objective here will be to develop a system which will provide a 24-hour mass average, and provide a sample for chemical analysis for trace elements, sulfate, nitrate, etc. by techniques such as x-ray fluorescence and ion chromatography. The second approach will be to investigate the feasibility of developing a piezoelectric personal mass monitor. This latter approach has the potential of providing short term exposures to fine particles. 
The design of the filter sampling device will be of first priority. Current options include a simple filter with a head for coarse particle cut-off, a 90-degree type virtual impactor with a dead space (or slow moving air space) for collecting coarse particles, a cyclonic head and a tandem (series) filter arrangement for coarse and fine particles.

The major problem anticipated is in the mass determination. The most advanced miniature pumps are limited to $21 \mathrm{iter} / \mathrm{min}$ and the total volume sampled in 24-hours is likely to be in the order of 2 cubic meters or less. For a 24-hour mean loading of $25 \mu \mathrm{g} / \mathrm{m}^{3}$ (which would be typical for ambient levels), only roughly $50 \mu \mathrm{g}$ would be collected. A reasonable gravimetric precision attainable with a microbalance is 20-30 $\mathrm{\mu g}$ for a 37-47 mm diameter millipore or nucleopore (ungreased) filter. A $300 \mu \mathrm{g}$ loading would be required to obtain a precision in the order of $\pm 10 \%$. Longer sampling times would increase the loading, and trade-offs may be required between sampling time and precision.

One proposed resolution to this problem is the use of a small sampling area. If the loading normally deposited on a $37 \mathrm{~mm}$ diameter filter can be deposited on a much smaller area, such as $1 \mathrm{~cm}^{2}$; the loading (in terms of $\mathrm{mg} / \mathrm{cm}^{2}$ ) could be increased by factor of greater than 10. This may be done either with a small filter or by the use of particle collimation. The application of beta attenuation (beta gauge developed by Lawrence Berkeley) for mass measurement depends on the density of the loading, and this approach appears promising for the small filter area. A beta gauge precison of less than $5 \mu \mathrm{g}$ is predicted based on the precision attainable with the larger filters. $\mathrm{A} 1 \mathrm{~cm}^{2}$ area filter would also be compatible with $x$-ray fluorescence, for which the sensitivity also depends on the density of the loading. Lawrence Berkeley has also developed a promising technique for small masses on filters based on correlations of mass with optical attenuation of visible light.

The major research problem anticipated with the piezoelectric mass sensor is the collection efficiency for very fine particles. In any design conceivable now, there must be some air flow past the quartz crystal, which does not act as an absolute filter. Commerical piezoelectric mass monitors are available, and use electrostatic precipitators to obtain high collection efficiencies for small particles. However, it may not be feasible or practical to design a personal type:collector which uses a precipitator charge up to several kilovolts.

We propose to investigate the feasibility of two approaches. One approach would be to impact the particles through a high velocity nozzle onto a crystal coated with a sticky substrate. The objective would be to obtain a collection efficiency which can be reasonable well-defined. A second possible approach is to employ thermal precipitation techniques. A thermal precipi-tator would have the following advantages: much lower requirements than the electrostate precipitator, slow flow-rate requirements, and high collection efficiency for fine particles. 


\section{Toxic Organics}

Little work has been done to this date on detailed evaluation of personal exposure devices for organics at ambient levels. There is also considerable uncertainty on priorities for specific organics or classes of organics. For these reasons, work during FY-79 will be limited to preparation of a survey to determine the nature of the problems, the most promising analytical approaches, and the pollutants which should be of highest priority. This survey will provide a report that will serve as the basis for an FY-80 work plan for evaluation of personal exposure monitors for toxic organics. Work in this area will then commence in FY-80, based on the established milestones having EPA and NBS concurrence.

\section{Project Milestones and Estimated Completion Dates}

1. Nitrogen Dioxide

- Completion of sample manifold and static test chamber for evaluating $\mathrm{NO}_{2}$ devices. (Completed)

- Evaluation of the Palmes diffusion tube passive device for $\mathrm{NO}_{2}$. (Completed)

- Preliminary investigation of membrane-backed diffusion devices as passive sensors for $\mathrm{NO}_{2}$. (Completed) 8/79

- Comparison of the standard chemical method for collected $\mathrm{NO}_{2}$ with ion chromatography. (Completed)

- Complete evaluation of filter (Millipore) diffusion barrier samplers for $\mathrm{NO}_{2}$ (except for wind tunnel tests). (Completed)

- Complete wind tunnel evaluation of Palmes diffusion tube sampler, and draft reports and publications on evaluation of Palmes diffusion tube sampler and filter diffusion barrier samplers. 


\section{Particulates}

- Complete literature review of possible sampling heads and collection techniques, and laboratory visits.

(Completed)

- Design wind tunnel for personal sampler investigations. (Completed)

- Select and evaluate candidate sampling approaches. (Completed)

- Install and initiate calibration of test system (particle generators, wind tunnel, sizing instrumentation). (Completed)

- Evaluate and calibrate wind tunnel (system performance; velocity stability and profile; turbulence) without aerosol injection. (Completed)

- Complete wind tunnel and test system (particle generator, sizing instrumentation). (Completed)

- Evaluate commercially available pumps. (Completed)

- Design, construct and make preliminary tests of prototype filter head.

3. Toxic Organics

${ }^{0}$ Report on FY-79 survey and FY-80 work plan. (Completed) 9/79

- Set up generation system and sampler exposure chamber for high concentration range (10-1000 ppb) for toxic organics. (Completed)

- Obtain commercial samplers for evaluation and establish a priority for evaluation of toxic organics. (Completed)

- Develop optimum GC analytical procedure(s) and determine linearity, precision, and minimum detection limits (MDL) on liquid standards (benzene). (Completed)

- Initiate project to determine thermal desorption characteristics (e.g., desorption temperature, background, desorption efficiency) for alternate sorbents, such as Tenax-GC, Porapaks, Ambersorbs. 
- Establish definitive protocol for sampler evaluation (sample generation, exposure, extraction, calibration, and analysis).

0

Define problems and develop detailed plan for evaluation of samplers over low concentration range (0.01-10 ppb).

Project Progress-FY-1980

\section{(a) Project Synopsis:}

\section{Nitrogen Dioxide}

In work on $\mathrm{NO}_{2}$ personal exposure monitors (samplers), a detailed progress report on a static evaluation of the Palmes diffusion tube sampler and FY-1979 work on $\mathrm{NO}_{2}$ monitor evaluations was completed and submitted to the EPA Project Officers for this project. Initial work in FY-1980 concentrated on the design and evaluation of membrane diffusion barrier samplers. The Palmes device was shown to have marginal sensitivity for sampling periods of $24 \mathrm{hrs}$. or less, while membrane-type samplers were determined to have a potential for greater sampling rates. Specifically, the following activities were covered: (1) completed preliminary evaluation of West-type silicone membrane samplers; (2) since elution was found to be a problem with samplers in (1), two other membrane filter holders were designed; (3) relative sampling rates of the Palmes, West, and NBS (containing a Millipore filter diffusion barrier) samplers were tested and found to be approximately 1,8 , and $30 \mathrm{~cm}^{3} /$ min., respectively; (4) tests on the NBS sampler were initiated to determine reproducibility, linearity, precision, and other factors.

On $\mathrm{NO}_{2}$ personal exposure monitors, work efforts were next focused on evaluation of alternate sampler designs as a take-off from the Westtype silicone membrane sampler. Work on the latter type of sampler was discontined due to elution problems. In addition, erratic results with this type of sampler were observed recently by other workers. Using a Nuclepore filter holder containing either a Millipore cellulose-type or teflon filter diffusion barrier backed by a polyester mat impregnated with $\mathrm{NO}_{2}$ absorbent (triethanolamine) also produced erratic results. Millipore filters were evaluated due to their higher flow permeation rate. However, preliminary results using a Nuclepore polycarbonate filter backed by a perforated metal screen (coated with treithanolamine) were much more precise. Evaluation of this more promising sampler was continued. This sampler appears superior to both Palmes and West-type devices.

While work with Millipore filter samplers gave erratic results, the use of a Nucleopore filter backed by a perforated metal screen coated with triethanolamine gave more precise results. These findings can be illustrated by the tabular data given below and plotted in Figure 15. 
I. Millipore $(8 \mu \mathrm{m})$ :

\begin{tabular}{|c|c|c|c|}
\hline $\begin{array}{l}\mathrm{ppm} \\
\mathrm{NO}_{2}\end{array}$ & $\begin{array}{c}\text { Exposure } \\
(\mathrm{h} .)\end{array}$ & $\begin{array}{c}(\mathrm{ppm}-\mathrm{h}) \\
\text { Integrated } \\
\text { Exposure }(\mathrm{B})\end{array}$ & $\begin{array}{c}\text { Average Amount } \\
\text { Found }(A) \\
(\mu \mathrm{g})\end{array}$ \\
\hline $\begin{array}{l}0.252 \\
0.252 \\
0.134 \\
0.148 \\
0.095 \\
0.095 \\
0.095\end{array}$ & $\begin{array}{c}24.9 \\
26.9 \\
6.2 \\
4.0 \\
1.5 \\
2.42 \\
3.25\end{array}$ & $\begin{array}{l}6.27 \\
6.78 \\
0.831 \\
0.592 \\
0.143 \\
0.230 \\
0.309\end{array}$ & $\begin{array}{c}26.4 \\
24.3 \\
21.2 \\
17.6 \\
1.09 \\
2.10 \\
1.84\end{array}$ \\
\hline
\end{tabular}

II. Nucleopore $(0.8 \mu \mathrm{m})$ :

$\begin{array}{lcl}0.104 & 20.3 & 2.11 \\ 0.104 & 7.0 & 0.728 \\ 0.104 & 16.2 & 1.69 \\ 0.221 & 3.0 & 0.663 \\ 0.221 & 6.0 & 1.33 \\ 0.064 & 17.75 & 1.14 \\ 0.064 & 7.13 & 0.456\end{array}$

6.80

3.2

2.28

3.1

4.65

2.8

2.30

3.5

4.16

3.1

4.32

3.8

1.68

3.7

As can be seen from these results, the $A / B$ results were far more constant for Nucleopore than for Millipore. Results indicated that the Nucleopore sampler was more than thirty times more sensitive than the Palmes device. An alternate diffusion sampler for $\mathrm{NO}_{2}$ has also been devised that employs the DuPont Pro-Tek Organic Vapor device with the charcoal collector strip removed. A grid coated with triethanolamine was inserted in place of this strip. Preliminary data indicated this modified device to have a high sampling rate. Finally, an $\mathrm{NO}_{2}$ introduction system was constructed to allow $\mathrm{NO}_{2}$ to be introduced into the air stream of the wind tunnel to evaluate passive samplers under wind conditions. It employs a cylinder of $\mathrm{NO}_{2}$ and heated transfer lines and valves that permit $\mathrm{NO}_{2}$ to flow at a constant rate into a mixing chamber, where it is mixed with air and subsequently introduced into the wind tunnel. This $\mathrm{NO}_{2}$ introduction system will be used for final evaluation of the Palmes diffusion tube sampler.

Further testing was carried out using the Nucleopore sampler under static testing conditions. The final phase of work on the evaluation of personal exposure samplers for nitrogen dioxide will involve dynamic tests performed in the wind tunnel.

\section{Particulates}

In work on particulate personal samplers, a detailed NBS FY-1979 activities report ("Personal Ambient Aerosol Sampler") was prepared and submitted to the EPA Project Officers for this part of the project. In addition, the test system (wind tunnel and associated equipment) fabrication 
was completed, assembled, and calibrations were initiated. Specifically:

(1) various filters were calibrated for pressure drop and flow rates;

(2) a Spinning Disc Particle Generator has been calibrated; and (3)

calibration work was initiated on an optical particle counter and a

Berglund-Lui generator, using the NBS Particle Doppler Shift Spectrometer.

Ammonium fluoroscein was chosen from a number of possibilities as the most suitable aerosol material. This material is widely used, easy and safe to handle, and provides aerosols having desirable characteristics. Selection of an appropriate material for aerosol generation was a necessary prerequisite to series filter and inlet testing using aerosol injection. The wind tunnel was assembled, and preliminary flow calibration was completed. The wind tunnel blower location was changed, and a grid was added to improve the air flow profile. A dispersion of small particle size aerosol was tested in the tunnel using oil aerosol and an optical particle counter. A TSI piezobalance obtained from EPA was found to be insufficiently sensitive for these measurements. A preliminary cut test was run on the Tomb-Treaftis Impactor and series filters. Series filters were selected as the most promising avenue for further aerosol sampling testing. In addition, extensive flow tests were performed using various filter holders and filter materials to determine the combination having the lowest pressure drop.

Extensive weighing tests were made of clean filters. It was determined that relative humidity above $50 \%$ affected the filter weights. Use of a new weighing room for weighing of the filters (Zeflour [Ghia], Fluoropore [Millipore], and Nucleopore) gave precise weights to better than $\pm 10 \mu \mathrm{g}$. Series filter components were selected and cut tests were completed. Due to design factors, testing of the Harvard cyclone sampler cut point was found to be difficult, and testing was delayed until the absolute sampling efficiency of this sampler could be tested in the wind tunnel. In addition, extensive evaluations were performed on eight pumps (including Spectrex, Brailsford, DuPont, and Bendix) for electrical efficiency, power requirements, and flow fluctuations. These tests were performed for flow conditions that might reasonably occur for any given series filter or impactor unit under study. The Bendix BDX-60 (or BDX55) motor-pump unit was selected as the most compatable with the series filter chosen. An effective pulse dampener was developed and tested, since the commercial pump units (and the Harvard sampler pump) had unsatisfactory flow fluctuations. At this point, the project sampler pump and series filters were selected, and the wind tunnel made ready for fluoroscein aerosol dispersion testing.

Progress on the NBS personal dosimeter for particulates was presented at the 13th Aerosol Technology Meeting at the Harvard University School of Public Health in August 1980, with considerable interest shown by researchers and commerical firms alike. The demonstrated unit was essentially complete, including case, except for a characterized inlet or head which has been designed and placed under fabrication. Since flow rate was of a primary concern, the effects of flow rate were measured for various dosimeter characteristics, such as battery type, battery drain, filter lot, pressure drop, and filter loading. Options of battery type and voltage regulation schemes were examined for different measurement 
needs. It was determined that any anticipated filter loadings will not affect flow rate through the dosimeter, and it was confirmed that loading of the coarse filter does not affect the size cut characteristics. The present stacked filter appears as the most optimum sampling arrangement, owing to its simplicity, inexpensiveness, and its ability to provide samples for $x$-ray fluorescence analysis. Large particle (15 um) injection schemes were tested in the wind tunnel for losses and for test section. Particle and velocity profiles are being tested for two of these schemes. Next, inlets will be tested, including and screened filter faces, the $10 \mathrm{~mm}$ Harvard cyclone sampler, and designs by Walter John (Berkeley, CA) and Klaus Willeke (Cincinnati, OH). The pump and battery systems selected for the dosimeter are far more optimum than other sampling systems considered or used in practice, including the Harvard sampler, both in terms of flow constancy, extended sampling time, and compactness.

\section{Toxic Organics}

In the area of evaluating samplers for toxic organics, a detailed report ("A Survey on Research Needs on Personal Samplers for Toxic Organic Compounds") was Finalized and submitted together with a proposed FY-1980 work plan to the EPA Project Officers for this project. Work was then initiated in line with the developed detailed work plan, approved by the EPA Project Officers.

An exposure chamber was constructed to allow passive samplers to sample low concentrations of benzene (and other) organic vapors. The chamber design is similar to the one used for $\mathrm{NO}_{2}$ sampling. The main components included a cylinder con-taining the organic compound in air, a supply of pure air (for dilution of the organic), flow controllers and voltmeters, and a chamber in which the samplers could be placed.

Standard solutions of benzene in $\mathrm{CS}_{2}$ were prepared at concentrations of $12-100 \mu \mathrm{g} / \mathrm{ml}$. Analys is by gas chromatography (flame ionization detection) showed good linearity over this range. A benzene solution (ca. $2 \mu \mathrm{g} / \mathrm{ml}$ ) was prepared and found to produce adequate response with the flame ionization detector. Standards prepared at this concentration level $(2 \mu \mathrm{g} / \mathrm{ml})$ were deemed adequate for the analysis of commerical passive samplers (e.g. the DuPont sampler). Using a sampling rate of 86 $\mathrm{ml} / \mathrm{min}$ (DuPont value) it appeared possible that sufficient sample could be collected at 1 ppm level in 12 minutes; at the $100 \mathrm{ppb}$ level in 2 hours; and at the 10 ppb level in 20 hours. An electron capture detector was ordered for for work with halogenated organics identified for study (e.g. perchloroethylene).

The exposure chamber for organics provided a dilution capability of about 100:1 at flows up to 8 1iters/minute. It has been used with cylinders containing known concentrations of benzene in air to provide diluted mixtures for the testing of the DuPont Pro-Tek badge, a diffusioncontrolled sampling device. Results, measured in duplicate runs, are 
given in Table 20. The charcoal strip in each sampler was desorbed with $1 \mathrm{ml}$ carbon disulfide and the extracts compared with standards of benzene in carbon disulfide, using a gas chromatograph with flame ionization detection. A strip of charcoal adsorbent from an unexposed sampler was used as the blank. The data revealed good precision between duplicates, but larger discrepancies between runs. Comparison of the results obtained was made with results expected, based on data supplied by DuPont at a benzene sampling rate of $86 \mathrm{mI} / \mathrm{min}$. DuPont information indicated that these devices will give low results when the air velocity is less than $35 \mathrm{ft}$./minute. Calibration curves for benzene in carbon disulfide were prepared covering concentrations down to $0.7 \mu \mathrm{g} / \mathrm{ml}$ benzene. Since a survey of five different batches of carbon disulfide showed some batches to have a measurable interference peak at the benzene elution peak time, its purity must be examined prior to its use for measurements of low benzene concentrations.

Table 20. Collection Efficiency of Benzene on Commerical Personal Monitoring Badges

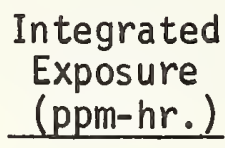

9.0

3.6

1.1

0.44
Amount Collected

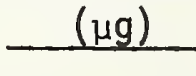

102

102

45.1

47.2

10.19

9.79

4.71

4.45
Amount

Expected

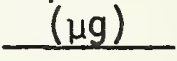

149

59.5

17.7

7.34
Collection Efficiency $(\%)$

69

78

56

62

(b) Low Velocity Wind Tunnel Facility for the Evaluation of Personnel Dosimeters

A low velocity wind tunnel was designed, constructed and characterized. The tunnel enables us to produce aerosol and gas samples of known component concentration and flow profiles for evaluation of the efficiencies of aerosol and gas sampling devices. The tunnel consists of a high efficiency absolute filter at the inlet, followed by a mixing grid to flatten the flow profile, and an injection section for the introduction of aerosol or gas samples. The test or sampling section of the tunnel (12 feet downwind of the injection section) has a 2-dimensional automated traverser, which enables us to accurately position and locate velocity or sample probes in order to measure the velocity and sample concentration profiles in the tunnel. The maximum velocity is $\simeq 3 \mathrm{~m} / \mathrm{s}$. The velocity profile is flat to better than 10 percent in the cross-section of the tunnel beyond the 2 inch wall boundary layer. There is very low mixing in the tunnel so that when a point-source injection system is used, we obtain sample profiles that are quite steeply parabolic. 
For multiple sampler testing we will need to use either multiple injection or more severe mixing to broaden the sample concentration profile.

We have used the wind tunnel to evaluate a personal ambient aerosol sampler (PAAS) that is currently under development at NBS. The two components of the PAAS--the aerosol sampling/ collection section and the sampling pump--have been evaluated separately. Eight different pumps have been evaluated for power requirements, pumping efficiency and flow stability. A pump has been selected that provides 5-6 1iters per minute for periods greater than the desired sampling period of 8 hours and has low power consumption (Bendix BDXX55). The pump was tested under load conditions on the sampler that exceed loading conditions normally found in field applications.

The aerosol sampler selected is a two stage series filter system using a $37 \mathrm{~mm}$ diameter, $8 \mu \mathrm{m}$ Nucleopore filter, followed by a high efficiency teflon filter. The sampler is to divide and collect ambient aerosol in two size fractions. The first filter ( $8 \mu \mathrm{m}$ Nucleopore) will collect respirable particles (about 2 to $15 \mu \mathrm{m}$ diameter), and the second filter (telfon) will collect the remaining smaller particles. The cutoff size (particle size at which the filter is 50 percent efficient) was determined for the Nucleopore filter using monodisperse aerosols generated by a vibrating orifice aerosol generator. Fluorescein aerosol was used to enable accurate spectrophotometric determination of low aerosol concentrations on the filters. The filter collection efficiency for the Nucleopore filter is presented in Fig. 16 as a function of particle aerodynamic diameter where aerodynamic diameter is a parameter that normalizes particles (regardless of shape) in terms of aerodynamic behavior for a unit density sphere. The collection efficiency for the first filter is defined as the aerosol concentration on the first filter divided by the sum of the concentrations on the first and second filter (total aerosol collected by the sampler). The sampler has the desired cut-off point of 2-3 um which does not vary significantly with flow rate in the range from 4 to 6 liters per minute. 


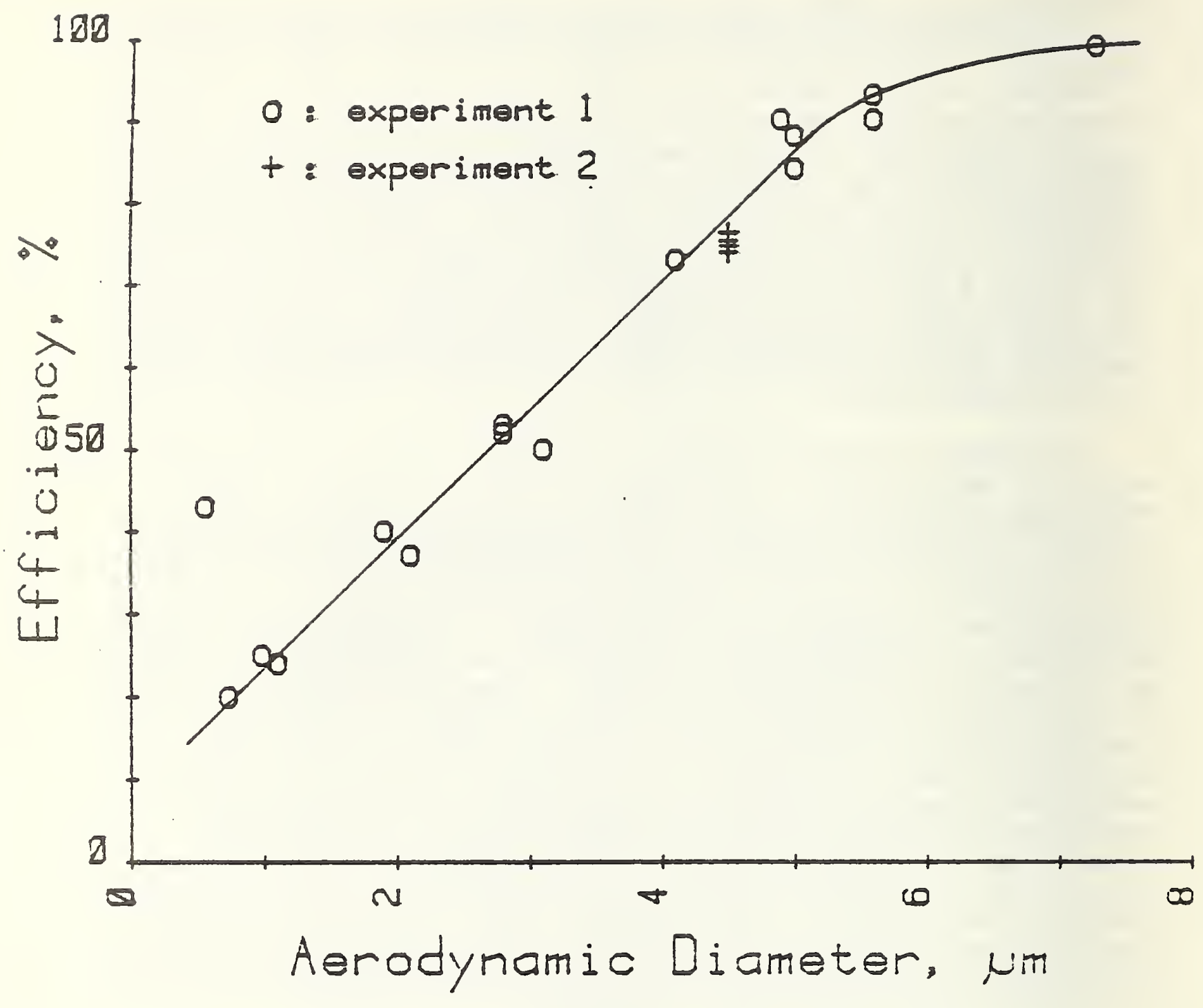

Figure 16. Coarse filter collection efficiency vs. particle size. 
(2) CALIBRATION AND MEASUREMENT TECHNIQUE DEVELOPMENT FOR UV RADIOMETRY

Participants: H. Kostkowski, R. Saunders

\section{Scope:}

The NBS will develop a high wavelength-accuracy spectroradiometer and a facility at NBS for characterizing and calibrating spectroradiometers, for the measurement of terrestrial irradiance from direct sunlight between 290 and $320 \mathrm{~nm}$ with an estimated uncertainty not exceeding $5 \%$. The NBS will use the above facility to develop techniques for determining the responsivity function for double monochromators in the 290 to $320 \mathrm{~nm}$ region required for the above stated solar measurements. A ring dye laser will be utilized for maximizing the $\mathrm{cw}$ power available in the 290 to $320 \mathrm{~nm}$ region, so that a data point in the extremely low level wings of the responsivity functions may be obtained with an accuracy of a few percent, with integration times considerably less than the one hour currently estimated. In addition, the facility will be set up in a separate laboratory dedicated to spectroradiometer characterizations, insuring greater flexibility and responsiveness to future needs of the EPA and the UV-B radiative research community.

The above accomplishments will make it possible for NBS to carry out the following:

0

Use the high wavelength-accuracy spectroradiometer in the summer of 1980 to determine the terrestrial irradiance from direct sunlight between 290 and $320 \mathrm{~nm}$ at Gainesville, Florida, in order to develop and illustrate optimum procedures for such measurements. These measurements will then be correlated with the amount of atmospheric ozone at the measurement site.

0

Characterize and calibrate two spectroradiometers selected by EPA to be used for direct or hemispherical terrestrial solar irradiance measurements or measurements of laboratory sources in the 290 to $320 \mathrm{~nm}$ region with an estimated uncertainty not exceeding $5 \%$, providing that the spectroradiometers and laboratory sources are sufficiently stable.

0

Complete first draft of a guide for performing accurate UV spectral irradiance measurements of the sun and of various laboratory sources, and provide consultation to EPA-supported UV $-B$ radiation effects researchers. 


\section{Project Milestones and Estimated Completion Dates}

1. Complete design and procurement of high wavelength accuracy spectroradiometer and laser systems. (Completed)

2. Set up test facility and initiate development of techniques for accurate determination of spectral responsivity functions in the spectral region from 290 to $320 \mathrm{~nm}$. (Completed)

3. Completion of UV-spectroradiometer and the facility at NBS for characterizing and calibrating spectroradiometers. (Completed)

4. Complete development and characterization of the new state-of-art spectroradiometer. (Completed)

5. Test the new instrument and measurement techniques and conduct filed measurements at Gainesville, Florida. (Completed)

6. Complete all data reduction and papers describing new techniques and results.

7. Perform additional experiments to address new information derived from the June 1980 field study; provide consultation and calibration services for EPA-designed spectroradiometers; finalize facility plan at NBS for solar measurements by the advanced spectroradiometer and measurement techniques.

FY-1981

\section{Project Progress-FY-1980}

\section{Project Synopsis:}

Initial FY-1980 activities focused on completion of the characterization laboratory and the computer-controlled automation system, and planning for field measurements at Alex Green's facility in Gainesville, Florida. A significant discovery was made having direct application to these measurements, whereby it was demonstrated that the wings of the slitscattering function of a spectroradiometer are significantly less when measuring coherent light. It should be possible to use this result in solar measurements by looking at the sun directly (no telescope) utilizing an entrance slit of about $20 \mu$ in width.

Corrections due to scattering should then be significantly reduced. Alignment and calibration procedures will be more difficult. Nevertheless, initial reaction to the potential benefit of this discovery was considered significant. 
The necessary equipment for the spectroradiometer was obtained, and the instrument was assembled and characterized.

The new spectroradiometer and measurement techniques were tested and used in very successful field measurements conducted at Gainesville, Florida during June 1980. Extensive and excellent data were obtained during the field tests. Reduction and analysis of these data was initiated in August 1980. In addition, NBS participated in the "UV Radiation Epidemiology Workshop" held at the University of Pittsburgh.

A paper was prepared on the field measurements study in Gainesville, Florida, entitled "New State of the Art in Solar Terrestrial Spectroradiometry below $300 \mathrm{~nm}$ ". Reduction of the extensive data taken in the field study is in progress.

\section{Description of the New Spectroradiometer and Characterization Facility}

A high-wavelength-accuracy, automated, portable, photon counting spectroradiometer has been developed for improving the accuracy of measurements of terrestrial solar irradiance below $300 \mathrm{~nm}$. The instrument was designed by Prof. W. Fastie of Johns Hopkins University and a number of people in the NBS Spectroradiometry Group and was manufactured by Research Support Instruments, Inc., and the NBS Instrument Shops. The major new feature of the instrument is a Fastie-Ebert double monochromator made of Invar, and containing two holographic gratings having 300 grooves per mm. The low temperature-coefficient and high dispersion make possible a high wavelength accuracy. In spite of its short focal length of $12.5 \mathrm{~cm}$, the wavelength readout is $0.001 \mathrm{~nm}$, setability 0.005 $\mathrm{nm}$ and accuracy $0.02 \mathrm{~nm}$. The detector is a selected, bialkali photomultiplier cooled to $-20^{\circ} \mathrm{C}$. The photon counting system has a $10 \mathrm{~ns}$ pulse pair resolution. Output signals in total counts and counts per second along with wavelength, ambient temperature, and time are printed out as we11 as stored on an 8" floppy disc. Wavelength settings and integration times are also computer controlled. A specially designed aperture/filter system makes it possible to replace filters, diffuser or aperture stops rapidly and with a high degree of freedom required for accurate pointing at the sun. The instrument has been used in extensive solar observations betwen 287 and $340 \mathrm{~nm}$ where the output signal varied from a few counts to $10^{7}$ counts per second.

Our dye laser UV spectroradiometer characterization facility has been completed. The heart of the system is a 12 watt argon laser, dye laser and a frequency doubling section with crystals and temperature control to provide outputs continuously tunable between 285 and $317 \mathrm{~nm}$ with output power varying from 1 to 4 milliwatts in this wavelength range. The power actually available is about 1/10 that specified and the contractor is trying to remedy this situation. Nevertheless, it still has been possible to determine the responsivity function of the special UV spectroradiometer described above in the vicinity of $290 \mathrm{~nm}$ 
to $10^{-7}$ of its peak. In addition we were able to detect a significant wavelength asymmetry in this responsivity function and find a set of operating conditions for which it was largely eliminated. This was quite important for terrestrial solar measurements in the very steep ozone cut-off region below $295 \mathrm{~nm}$. The characterization facility was also used in FY 1980 for determining the responsivity of BRH's UV Hazard Monitor and will be used during the next fiscal year for determining the spectal responsivity of the new NBS photoelectric pyrometer now being developed, for characterizing other UV spectroradiometers for the EPA and for further investigations of slit-scattering functions in the visible where the dye laser provides continuously tunable lines with over a watt of power.

The UV photon counting spectroradiometer will be used to establish a new state of the art for terrestrial solar spectral irradiance measurements below $300 \mathrm{~nm}$. This work will be done in collaboration with Alex Green at the University of Florida, Gainesville. Higher wavelength accuracy, measurement of the slit-scattering function, spectral deconvolution, a state-of-the-art photon counting system and application of the measurement equation with respect to all the radiation parameters are expected to significantly improve earlier efforts and establish standard procedures for terrestrial solar measurements. In addition, the Dobson method usually used for ozone determinations will be compared with the newer Green method. We are now in the process of reducing and analyzing the large amounts of data taken during June 1980. Several publications are expected from this effort, including a chapter in the NBS Self-Study Manual (a comprehensive treatise on radiometry, detailed enough for self-instruction, and is being published one to several chapters at a time as an NBS Technical Note in the 910 series). 


\section{Background}

Due to the anticipated marked increase in fossil fuel fired power plant development, it is necessary to plan effectively for the development of pollutant gas analysis methods to provide data quality assurance in pollutant gas emission measurements. During the past two decades the analysis of gaseous substances has become critically important. Toxic gases impact the health of the environment, industrial workers, and the general public. At the beginning of this period, few methods of analysis were available that provided the required accuracy at the usually low concentration levels of interest. Of the methods developed during this period, almost all have been relative, depending on gas standards of various types to achieve the required accuracy of measurement. Regulatory guidelines of the U.S. Environmental Protection Agency for ambient air quality monitoring, data reporting and surveillance provisions (FR 27558, May 10, 1979) specify that gaseous standards used must be working standards certified by comparison to NBS gaseous Standard Reference Materials (SRM's). It is clear that maintenance of NBS competences for gas analysis and gaseous SRM development represents a national need for environmental monitoring and research. While NBS has devoted extensive efforts towards the development and production of gas standards, it has spent insufficient time on investigations of new and absolute methods of gas analysis. It is imperative that the established NBS competences in gas analysis and gas standards production be continued and improved upon to insure that present and future national needs for data quality assurance in toxic gas monitoring and research are met. Activities related solely to replenishment of the inventory of existing NBS gaseous SRM's will, by itself, be insufficient to maintain the existing competences in this NBS activity.

An effort is needed to investigate existing absolute methods of chemical analysis in order to assess their applicability to gas analysis. In addition, an effort should be directed toward refining classical methods of gas analysis (such as volumetric and gravimetric techniques) in order to apply them to low concentration gas measurements. Further, some effort should be expended in investigating totally new measurement principles to determine their applicability to gas analysis.

The principal NBS activity under this project for FY-1980 has been an intercomparison of cylinder standards containing atmospheric concentrations of $\mathrm{CO}_{2}$. The methods used for these standards by NBS, Scripps Institute of Oceanography, and NOAA-Boulder are gravimetry, manometry, and volumetry, respectively. Accurate $\mathrm{CO}_{2}$ standars are needed to assess changes in atmospheric $\mathrm{CO}_{2}$ levels, which have been found to be on the increase over the last 20 years. 
There is a national concern for increasing atmospheric $\mathrm{CO}_{2}$ concentrations due to anticipated increase in house" effect of $\mathrm{CO}_{2}$ in altering global climate. Scripps has provided a broad $\mathrm{CO}_{2}$ standards service to the $\mathrm{CO}_{2}$ measurement community over the years. ${ }^{2}$ Scripps has recently requested that NBS assume the $\mathrm{CO}_{2}$ standards responsibility by development and maintenance of SRM's for $\mathrm{CO}_{2}$ in air at atmospheric concentrations. A discussion of the progress ih this intercomparison and a related activity is given below.

Selected Technical Activities

1. Standards for Measurement of Atmospheric Carbon Dioxide

E. Hughes, G. Sleater

Since the early 1950s internationally accepted standards for the measurement of atmospheric carbon dioxide have been traceable to a single set of manometrically analyzed standards prepared by Dr. David Keeling of Scripps Institute of Oceanography (SIO). Expansion of the monitoring network and the necessity for assuring a continuing supply of standards of high accuracy prompted the National Oceanic and Atmospheric Administration, the Department of Energy, and the Environmental Protection Agency to involve the NBS in a program to produce SRM's equal in accuracy to the standards maintained by Keeling. The production such standards requires a method of preparation of laboratory primary standards that are both accurate and reproducible. An uncertainity of $\pm 0.1 \mathrm{ppm}$ for a standard containing $330 \mathrm{ppm}$ was considered the maximum tolerable error.

A unique microgravimetric technique was developed and a set of 14 standards varying in concentration from 290 to $370 \mathrm{ppm}$ were prepared. The gravimetric technique involves weighing the carbon dioxide contained in a "micro cylinder" on a semi-micro balance and accurately transferring it to a large cylinder to which a weighed amount of air is added. The internal consistency of the set was evaluate by intercomparing samples in the set with each other using both gas chromatographic and non-dispersive infrared techniques. Painstaking care was taken to assure the highes degree of precision of the measurements. The results showed that the average of the deviations of the samples from the predicted value was $\pm 0.02 \mathrm{ppm}$ and the standard deviation of the observed value relative to the predicted value was $0.09 \mathrm{ppm}$. These standards were then used to analyze three working secondary standard mixtures of $346.7,340.0$ and $333.3 \mathrm{ppm}$, respectively.

The three secondary standards were taken to SIO and were analyzed by that laboratory in common with their own primary standards. A comparison of the NBS and SIO results is shown in the data below: 
Sample

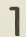

2

3
NBS Value

$346.7 \pm .17 \mathrm{ppm}$

$340.0 \pm .09 \mathrm{ppm}$

$333.3 \pm .10 \mathrm{ppm}$
Value Determined at SIO

$346.7 \mathrm{ppm}$

339.9 ppm

$333.2 \mathrm{ppm}$

The limits shown for the NBS value is an estimate of the uncertainty of the concentration of the secondary standard relative to the primary gravimetric standard. The uncertainty of the value measured by SIO is not known on an absolute basis but the repeatability of the measurement is estimated to be about $\pm 0.1 \mathrm{ppm}$.

The agreement is excellent considering the complete independence of approach of each laboratory. However, further work is planned to improve the precision of the measurements and thereby to determine whether the slight differences are random errors or biases between the methods. The most probable source of error in the NBS value would be in the analysis of the reagent gases while the most likely source of error at SIO would be associated with volume calibrations. Both of these error sources are being investigated.

Intercomparison of $\mathrm{O}_{3}, \mathrm{NO}$ and $\mathrm{NO}_{2}$ Measurement Standards by Gas Phase Titration

J. A. Hodgeson, A. Fried

Ozone $\left(\mathrm{O}_{3}\right)$, nitric oxide ( $\left.\mathrm{NO}\right)$, and nitrogen dioxide $\left(\mathrm{NO}_{2}\right)$ are critical constituents involved in the chemistry and physics of the troposphere and stratosphere and are subject to considerable monitoring requirements. For each of these gaseous constitutents, an individual measurement standard is currently employed for calibration. Ozone is measured on an absolute basis by absorption photometry using the known $\mathrm{O}_{3}$ cross section at the mercury $253.7 \mathrm{~nm}$ line. For $\mathrm{NO}$ and $\mathrm{NO}_{2}$ the measurement standards take the form of Standard Reference Materials (SRM's); $\mathrm{NO}$ is gravimetrically prepared in cylinders, while the $\mathrm{NO}_{2} \mathrm{SRM}$ is a permeation tube. This is the first concerted effort to intercompare gas standards by means of known chemical reactions, in this case employing the rapid, bimolecular reaction,

$$
\mathrm{NO} \text { (excess) }+\mathrm{O}_{3}=\mathrm{NO}_{2}+\mathrm{O}_{2} \text {. }
$$

At low concentrations of $\mathrm{O}_{3}$ (sub-ppm leve1s) and in the presence of excess $\mathrm{NO}$, the reaction is stoichiometric and the following relationship holds

$$
\Delta(\mathrm{NO})=\Delta\left(\mathrm{O}_{3}\right)=\Delta\left(\mathrm{NO}_{2}\right)
$$

Therefore a measurement of any one of the three components ( $\triangle(N O)$ = reacted, $\Delta\left(\mathrm{O}_{3}\right)=$ added, $\Delta\left(\mathrm{NO}_{2}\right)=$ produced) yields a measurement of the other two. 
In the experimental arrangement, NO from a standard cylinder (50 or $100 \mathrm{ppm}$ ) is diluted with clean air by a flow dilution ratio, $\mathrm{R}=30$ 100 , to obtain an initial NO concentration in the range of $0.5-1.5$ $\mathrm{ppm}$. The clean air stream passes through a variable $0_{3}$ source such that $0_{3}$ can be added in increments over the range, $0-1 \mathrm{ppm}$. The output of the reaction vessel passes to a sampling manifold where the concentrations are monitored with calibrated detectors.

Since the NO sample from the cylinder must be diluted, an accurate determination of the initial NO cylinder concentration requires an accurate determination of the flow dilution ratio, $R$. Considerable effort was expended in verifying the measurement of the NO and clean air flows. A wet test meter calibrated gravimetrically was employed for the air flow and the calibration of this meter was verifed at the Primary Flow Calibration Facility of the NBS Fluid Engineering Division. The low flow rate NO measurement was performed with a soap bubble meter, a mercury sealed piston device and a wet test meter, the accuracy of which was confirmed at the Primary Flow Calibration Facility. The estimated uncertainty in the measurement of $R$ is less than one percent.

Our initial objective was to provide an independent analysis of the NO SRM based on photometric $\mathrm{O}_{3}$ measurements. Our initial gas phase titration (GPT) measurements, however, gave NO cylinder analyses which were consistently 4 - 5 percent lower than the gravimetric analysis. This in turn indicates that the $\Delta\left(0_{3}\right)$ photometric measurement is less than $\Delta(N O)$ based on gravimetric calibration by the same amount. In an attempt to resolve this bias, a specially designed optoacoustic detector was constructed to measure the $\mathrm{NO}_{2}$ produced. This detector uses an argon-ion laser for excitation of the $\mathrm{NO}_{2}$ visible absorption band. The detector is calibrated with an $\mathrm{NO}_{2}$ permeation tube standard and has a minimum detection limit of .005 - .01 ppm. An important feature of the optoacoustic detector is that it does not respond to NO. Previous attempts to make similar measurements have used chemiluminescence NO detectors $\left(\mathrm{NO}_{\mathrm{X}}=\mathrm{NO}+\mathrm{NO}_{2}\right)$ which measure $\mathrm{NO}_{2}$ differentially, i.e. $\mathrm{NO}^{\mathrm{X}}$ $\mathrm{NO}=\mathrm{NO}_{2}$. Cồsiderable measurement uncertainty is introduced when $\mathrm{N} \delta$ is present ${ }^{2}$ at higher concentrations, as in the GPT experiment.

A limited number of measurements have been made thus far on $\mathrm{NO}_{2}$ produced, based on a permeation tube calibration, $\Delta\left(\mathrm{NO}_{2}\right) \mathrm{pt}$, but these data are revealing. A considerable amount of data have been obtained comparing $\Delta(\mathrm{NO})$ gravimetric, $\Delta(\mathrm{NO})$, and $\Delta\left(\mathrm{O}_{3}\right)$ by photometry $\left[\Delta\left(\mathrm{O}_{3}\right)_{\mathrm{ux}}\right]$ over the range $0-1 \mathrm{ppm}$. These data were obtained over a one year utime scale by two independent operators and comprise 15 different GPT experiments with 44 valid data pairs. These data sets can be fitted by linear-regression to yield the following relations:

$$
\begin{aligned}
& \Delta\left(\mathrm{O}_{3}\right)_{\mathrm{uv}}=(9.957 \pm 0.002) \Delta(\mathrm{NO})_{\mathrm{g}}+(.0002 \pm .001), n=44 \\
& \Delta\left(\mathrm{NO}_{2}\right)_{\mathrm{pt}}=(1.01 \pm 0.01) \Delta(\mathrm{NO})_{\mathrm{g}}-(.0052 \pm .0055), n=8
\end{aligned}
$$

The intercepts are negligible within the uncertainty of the fit. The precision of the GPT measurements is excellent and reflects the precision of the flow measurements as well as the stability of the 
various detectors. The apparent agreement between $\Delta(\mathrm{NO})$ and $\Delta\left(\mathrm{NO}_{2}\right)$ verifies the reaction stoichiometry and that the GPT apparatus is working properly. We are left with the implication that the $0_{3}$ ultraviolet measurement may be low by approximately 4 percent. This is a larger bias than can be explained by estimates of the uncertainty in the presently accepted value of the $\mathrm{O}_{3}$ cross-section. We are continuing the $\mathrm{NO}_{2}$ measurements for further verification of the above results. In addition, a new and improved $\mathrm{O}_{3}$ photometer is being constructed to replace our existing system and an independent $0_{3}$ calibration will be available soon. 
Background

In order to meaningfully address the national concerns for human health and environmenal impacts associated with assessments of energy-rellated air particulate emissions due to increased conventional and advanced fossil fuel technologies, improved and new methods technology is urgently needed to reliably characterize the chemical nature, particulate size, and anthropogenic contribution of air particulates. The EPA in its Research Outlook 1978 (EPA 600/9 78-001) identified aerosols as a high priority research area, second only to toxic substances. To illustrate, increased coal combustion is expected to contribute greatly to sulfur and nitrogen oxide emissions during the next 15 years. These gaseous emissions are precursors to higher toxicity secondary pollutants, such as sulfate and nitrate air particulates. In addition, human health impacts are of high concern since large size aerosols may be captured by sinus and throat mucus membranes and affect the digestive tract; while small size aerosols and fine particulates may be carried deep into the lungs with serious health consequences (e.g., emphysema, lung cancer). Further, increased particulate emissions are implicated in the deterioration of visibility and dramatic regional consequences of "acid rain," which episodes appear to be increasing in frequency. It is hence not suprising that increased attention is being given to methodology research to improve the characterization and quantitation of air particulate emissions.

\section{Projected Milestones}

In FY-1980, NBS activities under this Task Project (supported exclusively by the E/E EPA-NBS Program) have included:

- Development of the NBS Particle Doppler Shift Spectrometer for aerosol size measurements and characterization of aerosol generators.

- Development and characterization of an instrument for real-time analysis of sulfate aerosols.

${ }^{0}$ Development of low-level ${ }^{14} \mathrm{C}$ measurement and ${ }^{14} \mathrm{C} /{ }^{12} \mathrm{C}$ measurement methodology for assessments of biogenic versus fossil carbonaceous pollutant sources.

- Measurement methodology development for characterization of polycyclic aromatic hydrocarbons on air particulates. 
1. Particle Doppler Shift Spectrometer (PDSS)

D.S. Bright, R.A. Fletcher, I. Chabay, R.L. McKenzie

A vacuum sample tube was designed and installed in the PDSS in order to extend particle size measurements into the submicron range. Although the vacuum requirements are not extreme (down to a few torr), the stability of the system in holding a vacuum is critical due to the dependence of the slip correction and mean free path upon pressure. Extensive leak testing has been completed during which several components of the system (a pressure gauge and two high vacuum valves) were found to be defective and were replaced. The system is now ready to begin meements of the slip correction and very low Reynolds number air flow phenomena, and of submicron aerosols.

Experimental studies of the extremely small particle-induced fluid flow phenomena (fluid flows of the order of $0.01 \mathrm{~cm} / \mathrm{s}$ ) have been completed, and a theoretical model has been developed through the exact solution of the Navier-Stokes equation in the low Reynolds number regime. The theoretical model agrees well with the experimental results. These studies are now being prepared for publication.

The PDSS has been used to help characterize the properties of several aerosol generators. Our experience with the spinning disk generator shows it to be useful for generating mono-dispersed dioctylphthalate aerosols in the size range greater than about 15 micrometers, but not as useful as the Berglund-Lui vibrating orifice generator in the less-than 15 micrometer range.

Studies have also been made on the properties of ammonium fluorescein for use as an aerosol material. Aerosols generated by the vibrating orifice from a solution of ammonium fluorescein are solid, spherical particles, and have optical properties that allow ready detection at trace levels by spectrophotometric techniques. They have very low volatility and a high melting point, making them suitable for electron microprobe and $x$-ray analysis. In addition, ammonium fluorescein is considered to be non-toxic. Measurement of the diameter of ammonium fluorescein particles generated using the vibrating orifice generator has demonstrated that the size of the aerosol can be predicted and controlled by the molarity of the fluorescein solution used in the generator.

\section{Measurement of Ambient Sulfur Aerosols By a New Fast Response Flame Photometric Instrument}

\section{R.L. McKenzie, D.S. Bright, R.A. Fletcher}

Work continued on the characterization and optimization of the pulsed precipitator/flame photometric detector (FPD) aerosol sulfate measurement technique. As indicated in the preceding report, we made an evaluation of the feasibility of doping the hydrogen gas with $\mathrm{SO}_{2}$ or ethyl mercaptan in order to increase the total FPD response into a range 
where the instrument is more stable, and to increase the sensitivity of the response. The sensitivity of the system is a function of the total signal, because the FPD response $(R)$ is nonlinear with sulfur concentra$\operatorname{tion}(s) ;\left(i . e ., R \propto[S]^{n}\right.$, where $n=1.8$, therefore Sens. $=d R$ $\mathrm{n}[\mathrm{S}])$. We have experimentally verified this prediction; viz., an increase in total sulfur concentration of a factor of 2.6 is predicted to give an increase in sensitivity of 4.7 , in good agreement with an observed increase of 5.1 .

An important limitation in the application of the FPD for airborne sulfate particulate measurement is the slow response time of the burner to changes in sulfur concentration. The rise time for 90 percent of full equilibrium response is about 15 seconds, and the fall time is 2.8 seconds. For a precipitator operating frequency of $0.2 \mathrm{~Hz}$ we can obtain only 60 percent of the potentially available response. This is equivalent to a loss in sensitivity of almost 50 percent. We therefore have looked for better burner designs. We recently evaluated a burner and detector system bui]t by Dr. Don Stedman at the University of Michigan and found that it was much faster ( $r i s e$ time $=1 \mathrm{sec}$; fall time $=0.2$ seconds). We will obtain this instrument through a collaborative study arrangement, evaluate the burner for sensitivity, and optimize it for application with the pulsed precipitator technique. Table 21 shows a comparison of the percent of full response vs. precipitator frequency for the instrument we have been using, and for the University of Michigan burner/detector.

Table 21

Recovery of Potentially Available Response vs. Precipitation Frequency

Frequency (hertz)

.2

.5

2.0

4.0
Percent of Full Response

\begin{tabular}{cc}
\hline Meloy $^{\mathrm{a}}$ FPD & U. of Mich. FPD \\
60 & 100 \\
17 & 85 \\
5 & 65 \\
2 & 30 \\
-- & 10
\end{tabular}

"In order to adequately describe materials and experimental procedures, it is occasionally necessary to identify commercial products by manufacturer's name or label. In no instance does such identification imply endorsement by the National Bureau of Standards nor does it imply that the particular products or equipment is necessarily the best available for that purpose. 
The development and characterization of the Pulsed Electrostatic Precipitator-Flame Photometric Detector (PEP-FPD) system for the measurement of ambient level aerosol sulfur concentrations has been completed this year and a final report to EPA is in the review process. The basis of the technique is the use of the PEP to selectively modulate the aerosol component of the sample while leaving the gas phase component unaffected, resulting in a signal from the detector (FPD) which has two separable components corresponding to the particle and gas phase sulfur concentrations. Because the FPD response to sulfur concentration is non- 7 inear (Response $\sim[S]^{n}$ where $n=1.5$ to 2 depending on various flame and flow parameters) the sensitivity of the FPD is a function of the total sulfur concentration in the sample. The following data shows the sensitivity (dI/dS) and sensitivity relative to sensitivity at $1 \mathrm{ppb}$ for our FPD.

\begin{tabular}{rrrr}
{$[\mathrm{S}](\mathrm{ppb})$} & & $\mathrm{dI} / \mathrm{dS}(\mathrm{amp} / \mathrm{ppb})$ & $\mathrm{dI} / \mathrm{dS}$ relative \\
\cline { 1 - 1 } 1 & & $1.42 \times 10^{-10}$ & $(1)$ \\
5 & $4.81 \times 10^{-10}$ & 3.4 \\
10 & $8.13 \times 10^{-10}$ & 19.4 \\
50 & $2.75 \times 10^{-9}$ & 32.7 \\
100 & $4.65 \times 10^{-9}$ & 110.8 \\
200 & $7.86 \times 10^{-9}$ & 187.3 \\
500 & $1.57 \times 10^{-8}$ & \\
1000 & $2.66 \times 10^{-8}$ &
\end{tabular}

One consequence of this operational characteristic is that it is possible to increase the sensitivity of the PEP-FPD system by increasing the total sulfur concentration in the flame. We have verified this by adding a constant concentration of $\mathrm{CH}_{3} \mathrm{CH}_{2} \mathrm{SH}$ to the hydrogen. A second consequence, however is a complication of data reduction when one is attempting to measure more than one sample component because the sensitivities are related to total sulfur concentration, not to sample component concentrations. We have solved this problem by developing a data acquisition/ reduction procedure that enables us to achieve simultaneous measurement of aerosol and gas phase sulfur concentrations with sensitivities of 0.2 $\mu \mathrm{gm} \mathrm{S} / \mathrm{m}^{3}$ and $1 \mathrm{ppb}$ respectively. Initial data comparison from an intercomparison study shows a very high correlation coefficient with data obtained by a dichotomous sampler-x-ray-fluorescence system.

We have shown the major limitation in response time of the technique is in the FPD. (The commercially available FPDs have $>60 \mathrm{sec}$ rise times and $\sim 1.5 \mathrm{sec}$ fall times.) We have completed characterization of a new FPD design which has symmetric rise and fall times of less than one second. This faster response time means the PEP-FPD system will be capable of real-time measurements of ambient $\mathrm{SO}_{2} / \mathrm{SO}_{4}$ concentrations under conditions where the concentrations are dynamic, such as in plume traverses. Additional research will be needed to improve the sensitivity and flow stability of the fast-flame FPD to its full potential, though it now is comparable to commercially available FPDs in those respects. 
L. Currie, G. Klouda

a. Applications

The use of miniradiocarbon measurements with 5-10 mg (carbon) environmental samples (gases, atmospheric particles, sediment) has continued with the objective of utilizing the natural ${ }^{14} \mathrm{C} /{ }^{12} \mathrm{C}$ ratios to distinguish between biogenic and fossil pollutant sources. Important progress has been made in our understanding of the source of carbonaceous particles, especially of those collected in urban areas, through several cooperative studies with industry* and universities**. A critical element in these investigations has been the utilization of sample selectivity (chemical and physical) in combination with the radiocarbon measurements. That is, because small samples can be used, we have been able to increase the "information content" of our measurements by examining samples that have been preselected according to geographical region, known activities, chemical nature, and stable isotopic composition. A summary of our recent radiocarbon results, together with an indication of these critical supporting data, is given in Figure 17. The general conclusions to be drawn from these studies are (i) urban particles tend to show predominately fossil carbon (though with a non-negligible biospheric contribution); and (ii) particles from remote locations contain less organic carbon, which is primarily biogenic. An extremely important exception occurred in the Portland Study***, where the combustion of biospheric material (field, slash, and residential wood burning) led to the first observations of urban carbonzceous particles containing up to 100 percent biogenic carbon. These results have caused us to focus on the air pollution potential of residential wood combustion - a problem which will be a subject of our continuing studies (see Figure 18).

\section{b. Research Advances}

Research activities have been directed toward the analysis of atmospheric gases, high accuracy natural radiocarbon measurements, chemical-mathematical investigations of the Radiocarbon Dating Calibration Curve, and extension of the state of the environmental radiocarbon art to microgram samples using Accelerator Mass Spectrometry. Work with the atmospheric gases is still in the initial stages; thus far milligram quantities of atmospheric methane**** have been extracted for radiocarbon measurement. High accuracy radiocarbon measurements are underway in connection with calibration of the new NBS International Radiocarbon Dating Standard, and concomitant chemical-statisitical investigations have been performed in cooperation with the Australian National University on an International Laboratory Intercalibration exercise involving some 20 laboratories and three calibration materials (Oxalic Acid, 1850 Tree Rings, Sucrose). Evaluation of the fluctuations of the Radiocarbon Dating Calibration Curve is underway in cooperation with the Statitistical Engineering Division of NBS under the auspices of an international committee

\footnotetext{
* General Motors, GTobal Geochemistry Corporation.

** Oregon Graduate Center, Colorado School of Mines, University of Washington.

**** The Portland Aerosol Characterization Study measurements were carried out in cooperation with the Oregon Graduate Center and the Oregon Department, of Environmental Quality.

**** The methane study, of interest for climatic reasons and to learn about man's perturbation of the carbon cycle, is being carried out in .1 cooperation with NOAA (Air Reserves Lab).
} 


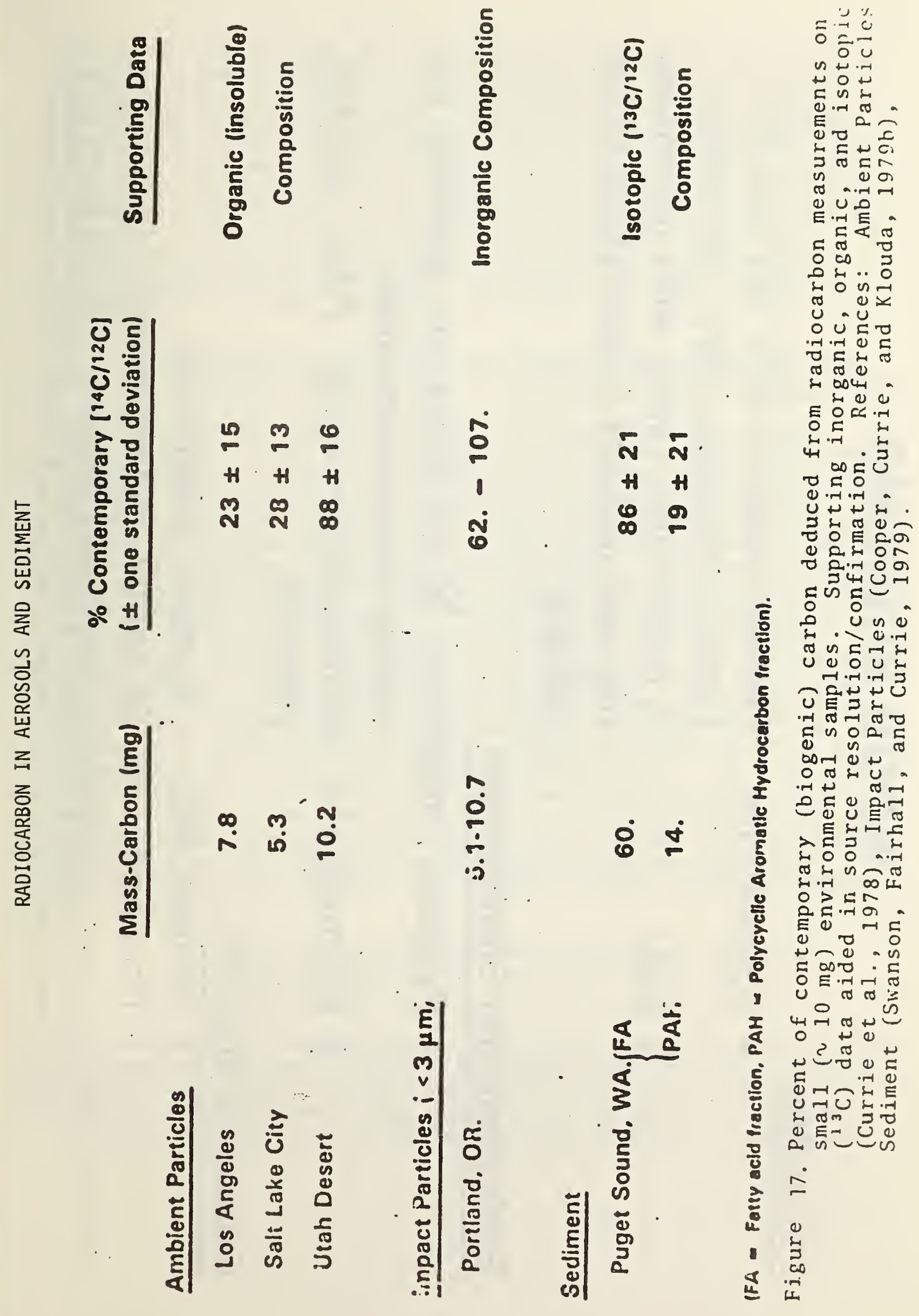




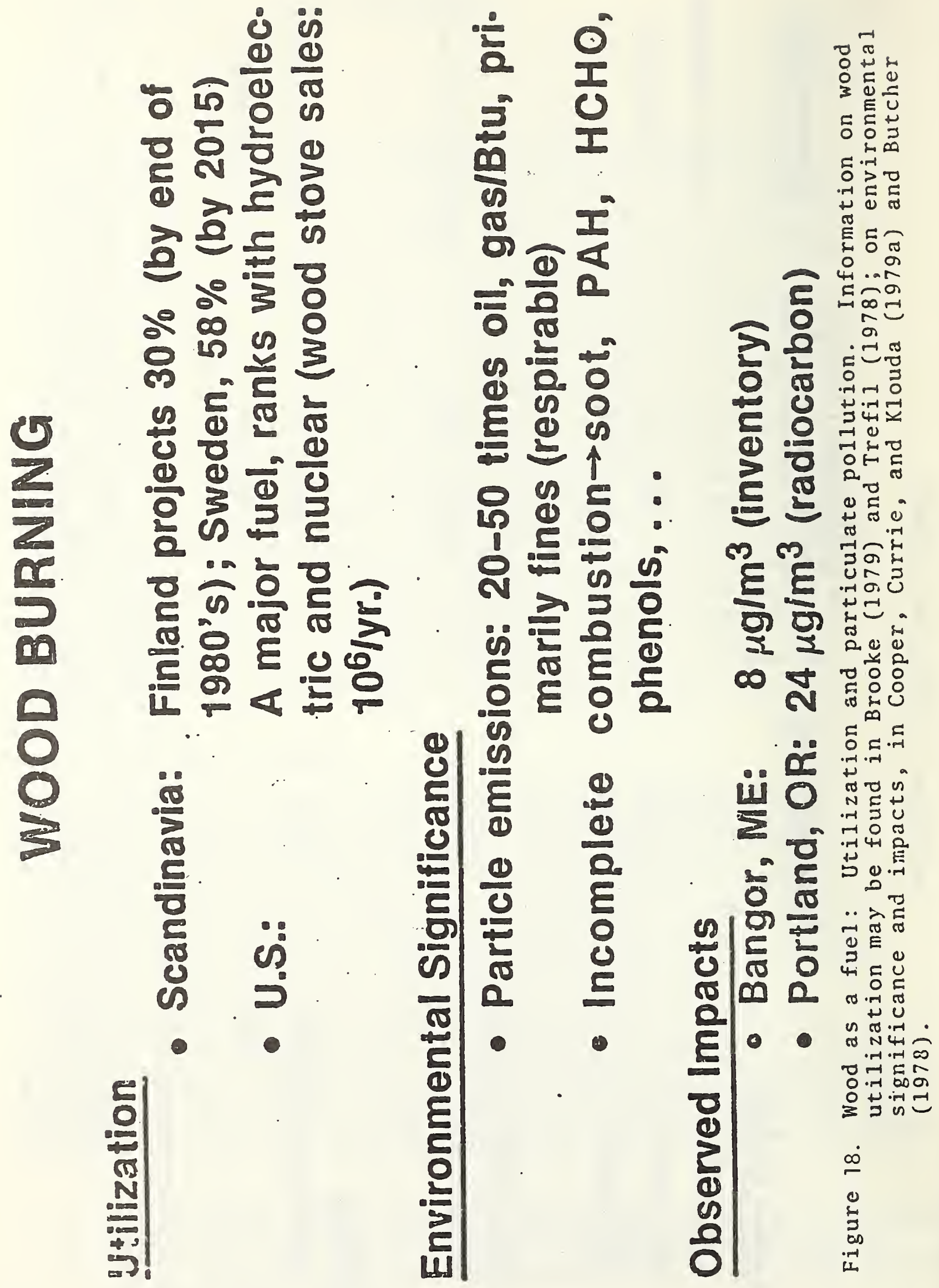


formed for this purpose. Information to be derived includes the assessment of accuracy, development of an accepted calibration curve for accurate dating, and geophysical model information for the assessment of past climatic variations. Studies initiated in 1979 show outstanding promise for the analysis of extremely "old" and extremely small samples. Our exploratory work, which utilized the NBS variable geometry isotope separator and the University of Rochester Tandem Van de Graaff Accelerator, demonstrated the feasibility of measuring natural radiocarbon in the 10 $\mu \mathrm{g}$ range. All of the above activities are intended to increase our ability to extract reliable information on anthropogenic and natural contributions to both environmental pollutants and chemically-induced climatic perturbations, now and in the past.

\section{c. Statistical Evaluations of the International Radiocarbon Cross Calibration Exercise, and of the Natural Radiocarbon Fluctuations}

High accuracy natural radiocarbon measurements are of critical importance in a number of fields, ranging from archeology to climatology to environmental science. In addition to our current activities related to the calibration of the new International Standard for Radiocarbon Dating (replacement for SRM 4990B), we have contributed to the numerical evaluation of interlaboratory data representing a) high precision measurements of homogeneous primary and secondary natural radiocarbon standards, and b) the "calibration" relationship between radiocarbon concentrations and calendar age as deduced from dendrochronology (tree ring measurements). The first activity is important for assessing the relative activity of the three "standards" and for identifying error sources and limits (random and systematic) among the best laboratories internationally. Deliberations on the second topic culminated in the organization of the International Committee for the Calibration of the Radiocarbon Time Scale, to which NBS scientist, Lloyd A. Currie, is an advisor. The fine structure of the radiocarbon dating calibration curve -- i.e., long-term trends and short-term fluctuations about the simple exponential decay model -- is important for two major reasons: a) fine structure must be adequately evaluated/fitted to achieve accurate dating, b) modeling of the fine structure provides a means for us to learn more about the history of the earth (climate, environmental changes, ocean circulation, solar activity, etc.).

\section{i. Exploratory Analysis of the International Radiocarbon Cross-Calibration Data: Consensus Values and Inter- laboratory Error}

Data from the international cross-calibration exercise have provided an important opportunity to assess the state-of-the-art of high-precision natural radiocarbon measurement and to derive consensus values for two relatively pure and homogeneous materials relative to the international radiocarbon dating standard (NBS 0xalic Acid, SRM-4990B). Performance 
was good: some fifteen laboratories returned results for the two independent ratios -- Australian National University Sucrose/Modern Standard (S/M) and 1850 Wood/Modern Standard (W/M) -- with a typical imprecision (standard deviation) of about five percent per mi11; and the distribution of results was relatively narrow. The primary limitations encountered were incomplete laboratory data and variable reporting practices.

Analysis of the data led to the following observations:

- Imprecisions (standard deviations) reported varied from about two percent per mill to eight percent per mill, with one laboratory reporting 28 percent per mill. Most, but not all, imprecisions represented counting statistics only.

- Apparent blunders (large deviations from consensus values) occurred in a few cases, especially for estimates of the ratio S/M.

- "Laboratory error," $\sigma_{x}$-- i.e., a non-Poisson error component --

was estimated from the data distributions for both sample ratios. Results were similar: for $S / M, \hat{\sigma}_{x}=3.5$ percent per mill; for $W / M, \hat{o}_{X}=1.8$ percent per mill. These error estimates, though rather uncertain because of the limited number of degree of freedom, are consistent with earlier estimates, and the may reflect the accuracy limit for current counting techniques.

- Consensus ratios, following the use of exploratory techniques to identify blunders, and taking into account "laboratory error" and varying Poisson imprecision, were $\Delta^{14} \mathrm{C}=508.1 \pm 2.0$ percent per mil1 (1850 Wood, age-corrected for decay to 1950). (Uncertainties represent one standard error, and both $S$ and $W$ are normalized to $\delta^{13} \mathrm{C}=-25$ percent per mi11).

Further results of the evaluation include $\delta^{13} \mathrm{C}$ distributions and the search for measurement method-, sample nature-, and isotope fractionationrelated effects. (See Figure 19 for histograms of the intercomparison results).

\section{ij. Calibration of the Radiocarbon Time Scale}

Evaluation of the structure of the radiocarbon calibration curve is critically important both for accurate dating (as shown in Figure 20) and for geophysics and climatology (Figure 21). At the present time, because of the remarkable precision of natural radiocarbon measurements and the unique time ( 8000 year) record provided by dendrochronology, radiocarbon is the only radionuclide capable of giving us direct information on past fluctuations in solar activity. When combined with geophysical modeling and ice core measurements of the ancient atmosphere $\left(\mathrm{CO}_{2}\right)$, the ${ }^{14} \mathrm{C}$ variations will yield further insight into the history of the carbon cycle and prognosis for "the $\mathrm{CO}_{2}$ problem." 


\section{CROSS-CALIBRATION.HISTOGRAMS}

(corrected ${ }^{14} \mathrm{C}$ deviations, in \%)

\begin{tabular}{|c|c|c|c|c|c|}
\hline \multicolumn{2}{|c|}{$S / M$} & & & & \\
\hline 530 & q & \multicolumn{2}{|c|}{ W/M } & \multicolumn{2}{|c|}{ S/W } \\
\hline \multicolumn{6}{|l|}{52.5} \\
\hline 520 & & 10 & s & 525 & q \\
\hline 515 & $s$ & 5 & $q$ & 520 & $\mathrm{G} \mathrm{km}$ \\
\hline 510 & $A G k$ & 0 & d j $\mathrm{L}$ & 515 & 0 \\
\hline 505 & $E F^{\prime} L O$ & -5 & $A B E G h$ & 510 & $A E F$ \\
\hline 500 & $B d m R$ & -10 & F $k o$ & 505 & $B$ \\
\hline 495 & h j & -15 & $\mathrm{mP}$ & 500 & $d h L$ \\
\hline 490 & & & & 495 & $c j P s$ \\
\hline 485 & $I$ & & & & \\
\hline 480 & $P$ & & & & \\
\hline
\end{tabular}

- S, M, W = Sucrose, Modern Standard, Wood

- $\sigma_{\mathrm{i}}=2 \% 00-4 \% 00$ (Cap.); 5\% - 8\% (Lower Case)

Figure 19. Histogram of the radiocarbon ratios for ANU sucrose (S), 1850 wood $(W)$ and the modern standard (M; $0.95 \times$ NBS oxal ic acid). Numerical scales are expressed as deviations (in parts-perthousand) from unity from each of the three ratios; and laboratories are coded alphabetically. 


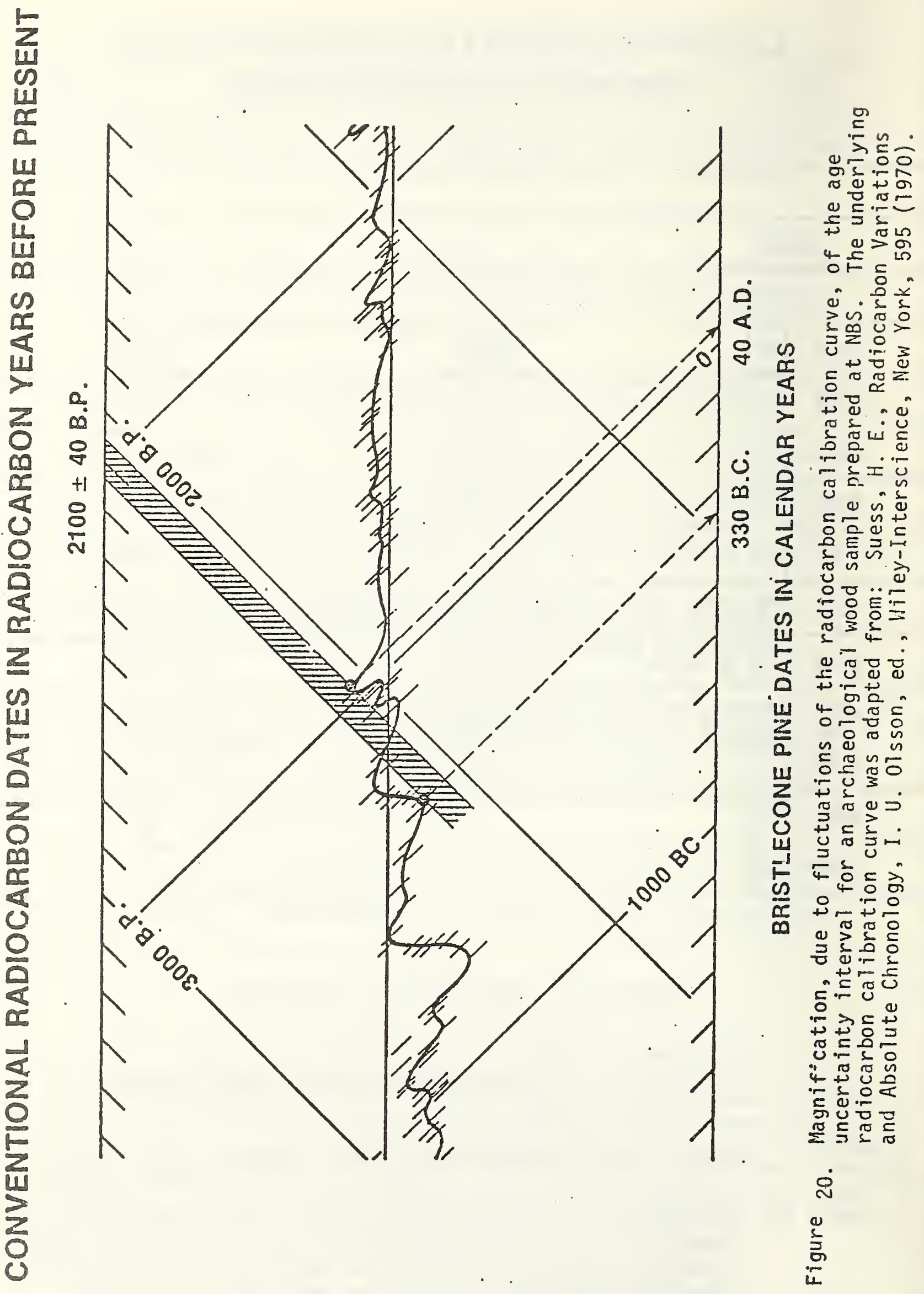



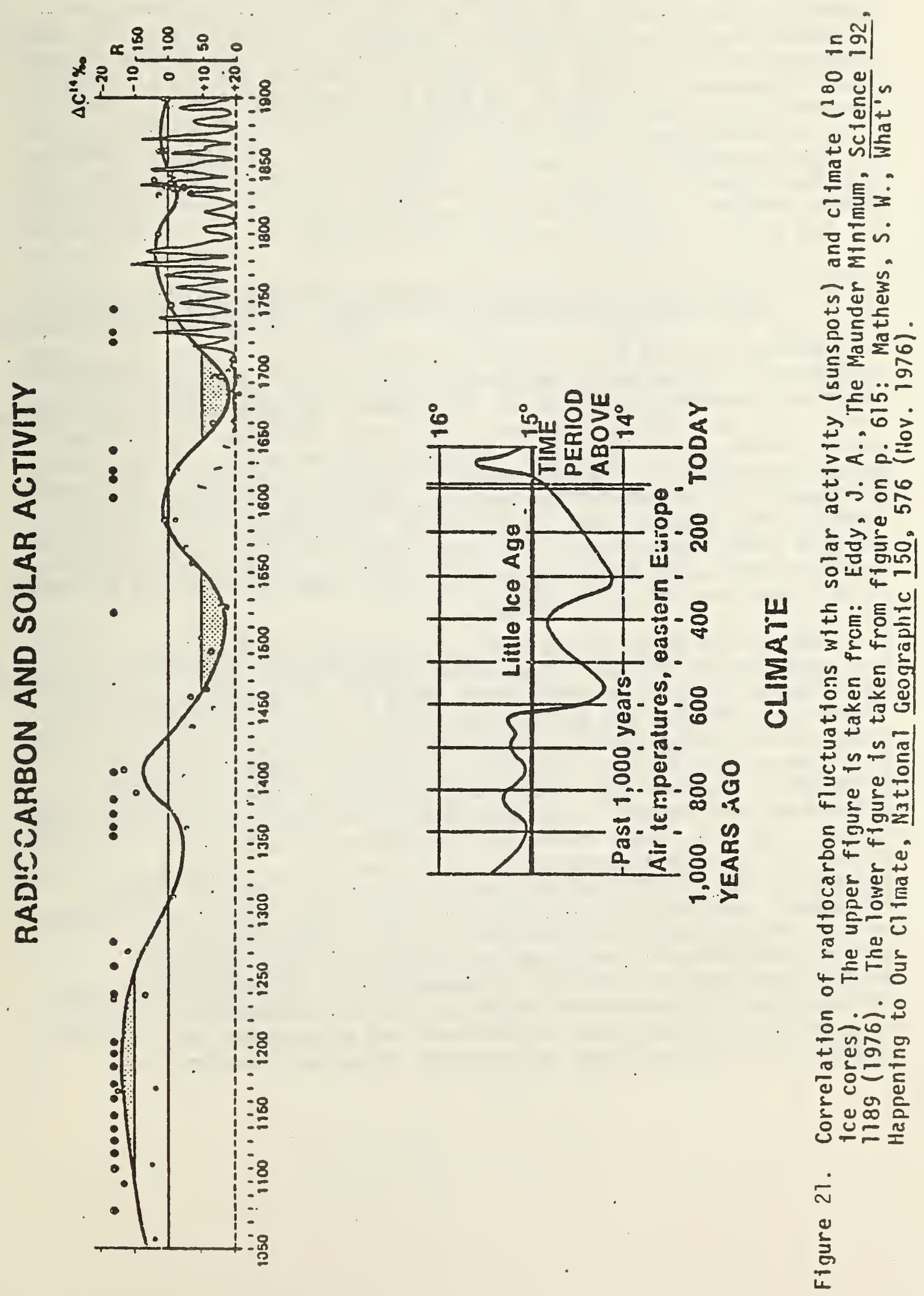
Objectives of the International Committee are to assemble high quality data and to utilize geophysical and statistical-mathematical approaches to "fitting" the data. More specifically, the Committee seeks to identify means for combining all (reliable) ${ }^{14} \mathrm{C}$ measurements of treering dated wood specimens into a single radiocarbon scale calibration scheme, preserving as much of the high frequency components as seem warranted by the data. During the past year cooperative efforts by L. A. Currie (Center for Analytical Chemistry), C. Spiegelman (Center for Applied Mathematics), and J. C. Lerman (Univ. of Arizona) have produced important insights into measurement and interlaboratory errors (see also part 1, above), assumed functional models, and best estimates for the calibration curve and its uncertainty band.

d. Advances in Small Sample Radiocarbon Measurement Techniques; Application to the Assay of Individual Atmospheric Chemical Species

During the past year the NBS program to utilize natural radiocarbon as a tracer for sources of carbonaceous pollutants has been directed toward a) fundamental advances in measurement and data evaluation techniques and b) critical studies of urban air pollution episodes (such as the Denver "brown cloud") and basic investigations of sources of atmospheric gases and particles. The importance of these investigations rests upon the impact of both natural and man-made carbonaceous contaminants on health, stratospheric ozone, and climate. The success of this program, the only such program extant, is due to the ability of NBS to perform reliable radiocarbon measurements on ambient samples containing microgram to milligram quantities of carbon.

Measurement advances are continuing through the development of miniature gas proportional counter and nuclear accelerator ("atom-counting") techniques which provide sensitivity gains of $10^{2}$ to $10^{5}$ compared to conventional radiocarbon dating techniques. Important meetings treating these topics (among others), organized by individuals in the group, included an ACS Symposium ("Nuclear and Chemical Dating Techniques", March 1980) and a meeting at NBS (August 1980) on sample preparation for accelerator measurements. Our work with the accelerator, which last year demonstrated the capability of precise measurement using less than $40 \mu \mathrm{g}$ (carbon) of the new international radiocarbon dating standard, is continuing with studies in alternative source preparation methods, isotopic enrichment, and baseline tests with the first "dedicated" tandem accelerator constructed exclusively for atom-counting measurements. This particular machine is considered an National Science Foundation Regional Facility, and it will be located at the University of Arizona. The accelerator approach will make possible the radiocarbon assay of ug quantities of individual hydrocarbons and halocarbons, and individual chemical and size fractions of non-urban atmospheric particulates. 
Applications to urban pollution and source studies of carbonaceous particles have centered on vegetative burning in the Portland, Oregon region, vegetative vs. $0 i l$ shale aerosol contributions in a remote Utah location, urban pollution in Los Angeles and Denver, and an international effort (joint US - USSR AFAEX-79 project) to investigate "processes and mechanisms of formation and transformation of natural aerosol" in a remote forested region in the Soviet Union. In all of these studies, which were broadly interdisciplinary in nature, our group provided the only isotopic data; and the natural radiocarbon results yielded the only unique measure of the biogenic (non-fossil) contribution. This was especially important in the Denver and Portland studies where as a result of our measurements it was concluded that residential wood burning constituted one of most significant sources of inhalable $(<2.5 \mu \mathrm{m})$ and visibility-reducing particles. In Denver for example, it was concluded that 18 percent of the visibility reduction of the winter time atmosphere arose from wood burning. Furthermore, 39 percent of the most effective visibity-reducing species -- elemental ("graphitic") carbon -- has been ascribed to wood burning.

It should be noted that the foregoing regional studies have included considerable data on chemical and physical characteristics of the ambient particles, together with supporting emission inventory and meteorological data. Although radiocarbon techniques provided the only direct measure of contemporary carbon, the other (indirect) indicators supported the conclusions from radiocarbon measurements in every case. A major challenge exists in selecting and evaluating the entire multidimensional data patterns from such experiments; this need formed the basis for the first Receptor Modeling Workshop (March 1980 in Durham) and the resulting committee on chemical mass balance and multivariate analysis methods of source reconciliation. Our program at NBS has been expanded to include such modeling efforts, in cooperation with NBS Center for Applied Mathematics. The combined use of isotopic datai $\left({ }^{13} \mathrm{C}\right.$ and $\left.{ }^{14} \mathrm{C}\right)$ with particle size and chemical data (pyrolysis mass spectrometry, inorganic composition and elemental vs. organic carbon) has, in fact, been an intrinsic part of the above urban and remote particulate studies.

A summary of our recent radiocarbon measurements, which now include atmospheric gases as well as particles, is given in Table 22 . Future work will be directed toward further sampling of selected gases (hydrocarbons, carbon monoxide), sources of urban run-off and sediment studies, high-altitude sampling, investigations of arctic haze and climatological consequences, and paleoatmospheric (isotopic) composition via ice core samples. 

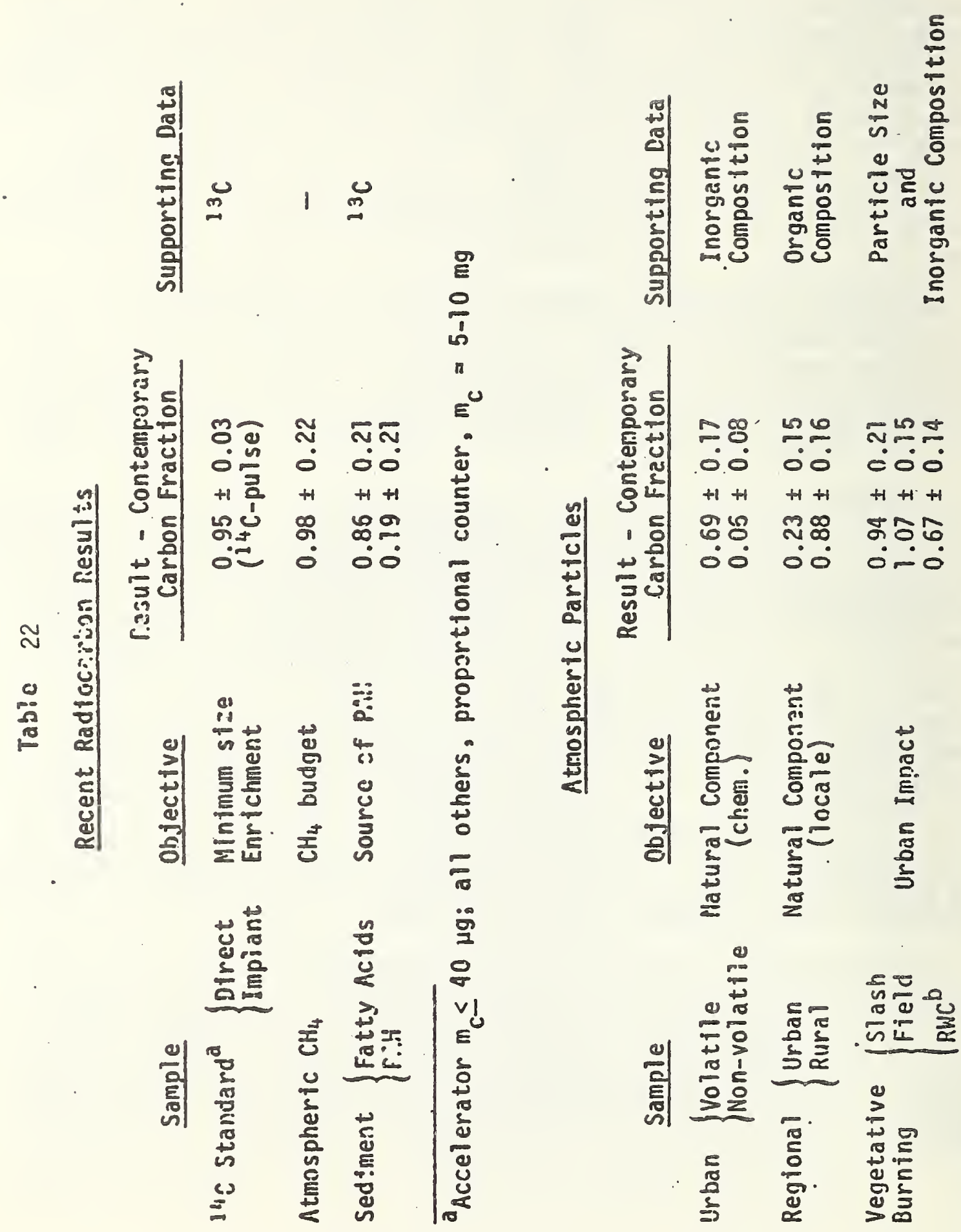

के
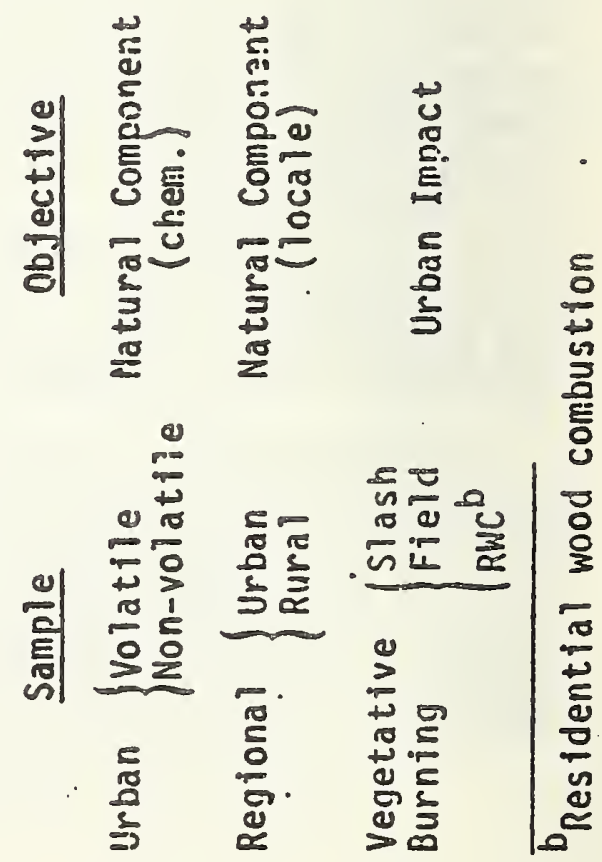


\section{Organics on Air Particulates}

Analytical methods were evaluated for the isolation, separation, and identification of polycyclic aromatic hydrocarbons (PAH) in urban air particulate matter. These methods have been employed for the qualitative characterization of the PAHs on SRM 1648 (Urban Air Particulate Matter).

An important part of this methodology is the use of normal phase high-performance liquid chromatography (HPLC) on an aminosilane column to isolate PAH fractions according to the number of condensed aromatic ring [Anal. Chem. 49, 2306 (1977)]. The normal phase liquid chromatogram of the analysis of the urban air particulates (SRM 1648) is shown in Figure 22. Each of the numbered fractions was then analyzed by gas chromatography/mass spectrometry (GC/MS) and reverse-phase HPLC with fluorescence detection for identification of the PAH. The GC analyses of fractions 3,4 , and 5 are shown in Figure 23. The isolation of PAH fractions in this manner allows identification of many minor components in the mixture which would otherwise be obscured. The PAHs identified in SRM 1648 are summarized in Table 23. Reverse-phase HPLC analyses of the fractions using fluorescence detection confirmed the identities of many of the PAHs. The liquid chromatogram of the analys is of fraction 4 is shown in Figure 24.

Several different organic solvents have been investigated for ultrasonic extraction of the PAHs from the particulate matter (i.e., cyclohexane, methylene chloride, methanol, and tetrahydrofuran). There are numerous reports in the literature of the use of cyclohexane for these extractions. In our studies the polar solvents appear to be more effective in removing the PAHs from these particulates. However, these solvents also remove additional organic compounds (i.e., those more polar than PAHs) which create additional problems in the isolation of the PAHs. The analytical methodology is being modified to solve these problems. 


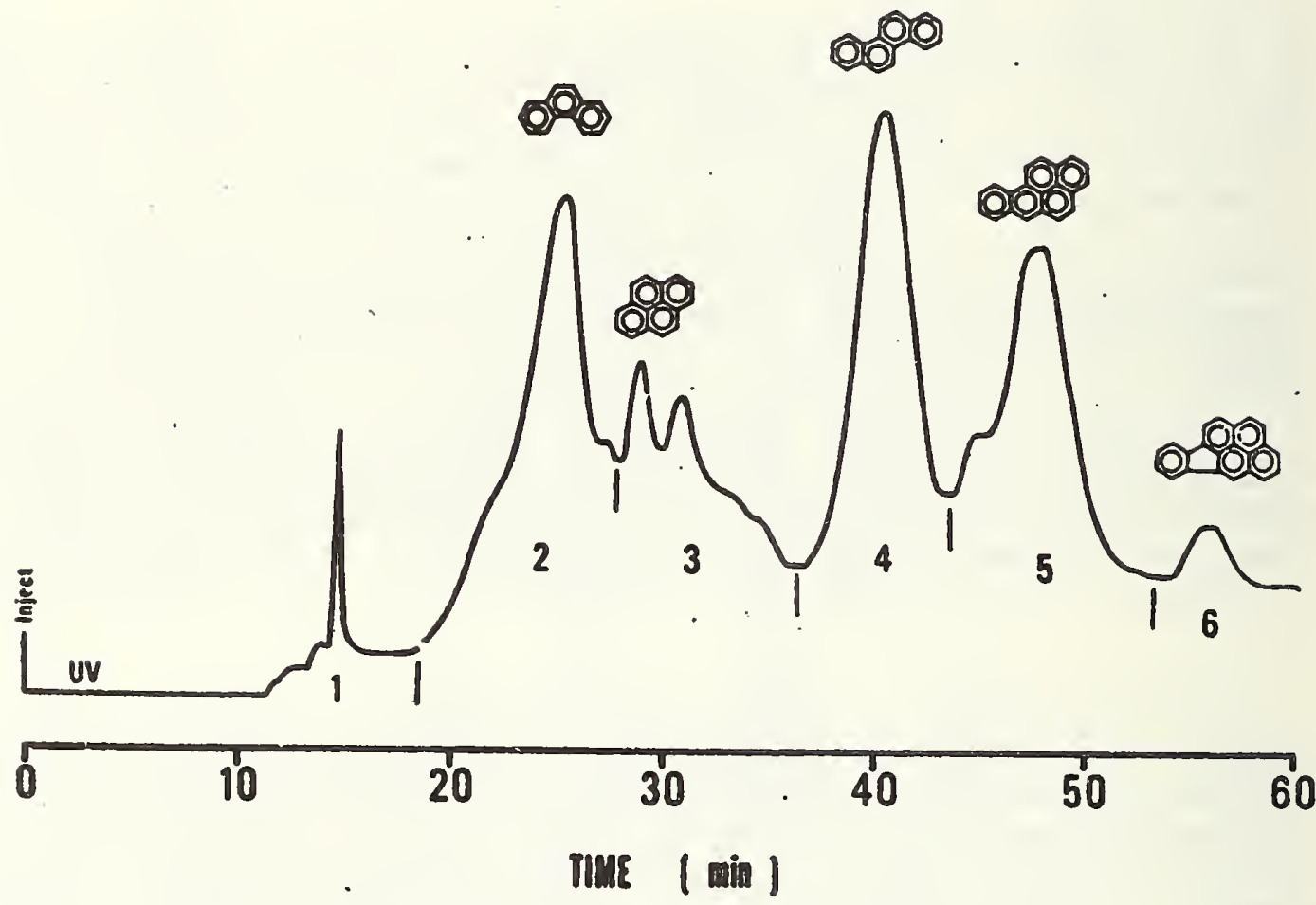

Figure 22. Normal phase liquid chromatogram of PAH extracted from urban air particulates. Column: semipreparative $\mu$ Bondapak $\mathrm{NH}_{2}$. Detection: UV absorbance at 254 $\mathrm{nm}$. Conditions: $\mathrm{n}$-hexane at $1 \mathrm{~mL} / \mathrm{min}$. Numbers indicate fractions collected for subsequent. analysis and the PAH structures indicate elution times for these representative compounds. 


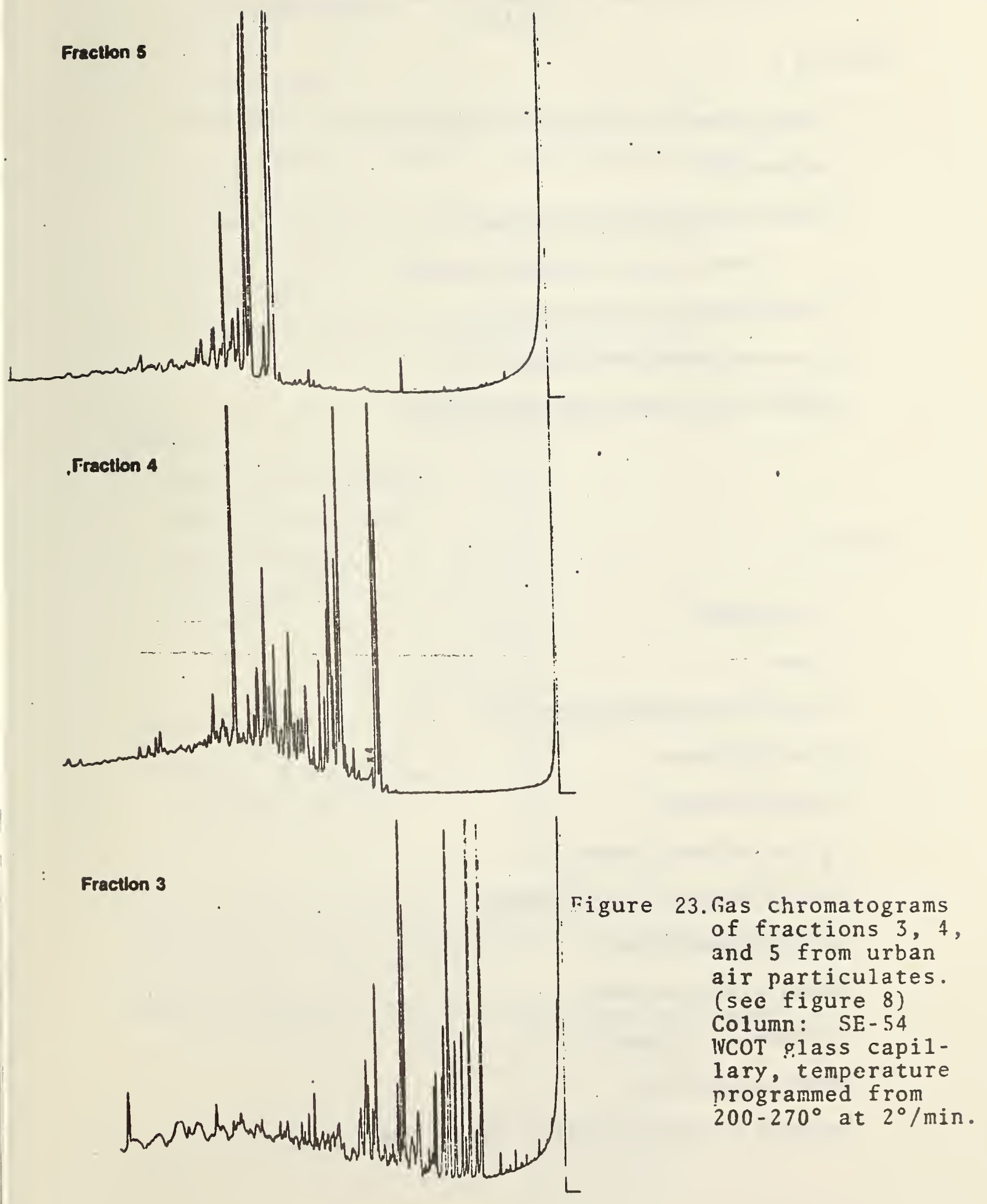

173 
PAH's Identified in Urban Air Particulate Matter (SRM 1648)

Fraction 2

\author{
Phenanthrene ${ }^{b}$ \\ Anthracene ${ }^{b}$ \\ Methy]phenanthrenes/anthracenes (4) \\ (probably methylphenanthracenes) \\ 4H-cyclopenta(def)phenanthrene \\ $\mathrm{C}_{2}$-phenanthrenes/anthracenes (6) \\ Methy 1-4H-cyclopenta(def)phenanthrene
}

Fraction 3

Fluoranthene ${ }^{b}$

Pyrene $^{b}$

Methylfluoranthenes/pyrenes (5)

Benzo[a]fluorene

Benzo[b]fluorene

$\mathrm{C}_{2}$-Fluoranthene/pyrenes (2)

Benzo[b]naphtho $[2,1-d]$ thiophene

Benzo[c]phenanthrene

Benzo[ghi]fluoranthene

Cyclopenta[cd]pyrene

Benzacenaphthylene

Unknowns of molecular weight 230 and 234 
Fraction 4

Benz[a]anthracene ${ }^{b}$

Chrysene $^{b}$

Triphenylene

Methylbenz[a]anthracenes/chrysenes/triphenylenes (3)

(1-methy1-, 2-methy1-, and 3-methylchrysene) ${ }^{b}$

Binaphthyls (3)

$\mathrm{C}_{2}$-benz[a]anthracenes/chrysenes/triphenylenes

Methylbinaphthyls (3)

Quarterpheny 1

Unknowns of molecular weight 240 and 278 (3)

Fraction 5

Benzo[b]fluoranthene ${ }^{b}$

Benzo[k]fluoranthene ${ }^{b}$

Benzo[e]pyrene ${ }^{b}$

Benzo[a]pyrene

Perylene

Fraction 6

Indeno $[1,2,3-c d]$ pyrene $^{b}$

Benzo[ghi]perylene ${ }^{b}$

adentities of specific isomers based on mass spectra and gas chromatographic retention time.

bIdentity confirmed by HPLC and fluorescence spectroscopy. 


\section{Urban Air Particulates - Fraction 4 Reverse Phase HPLC}

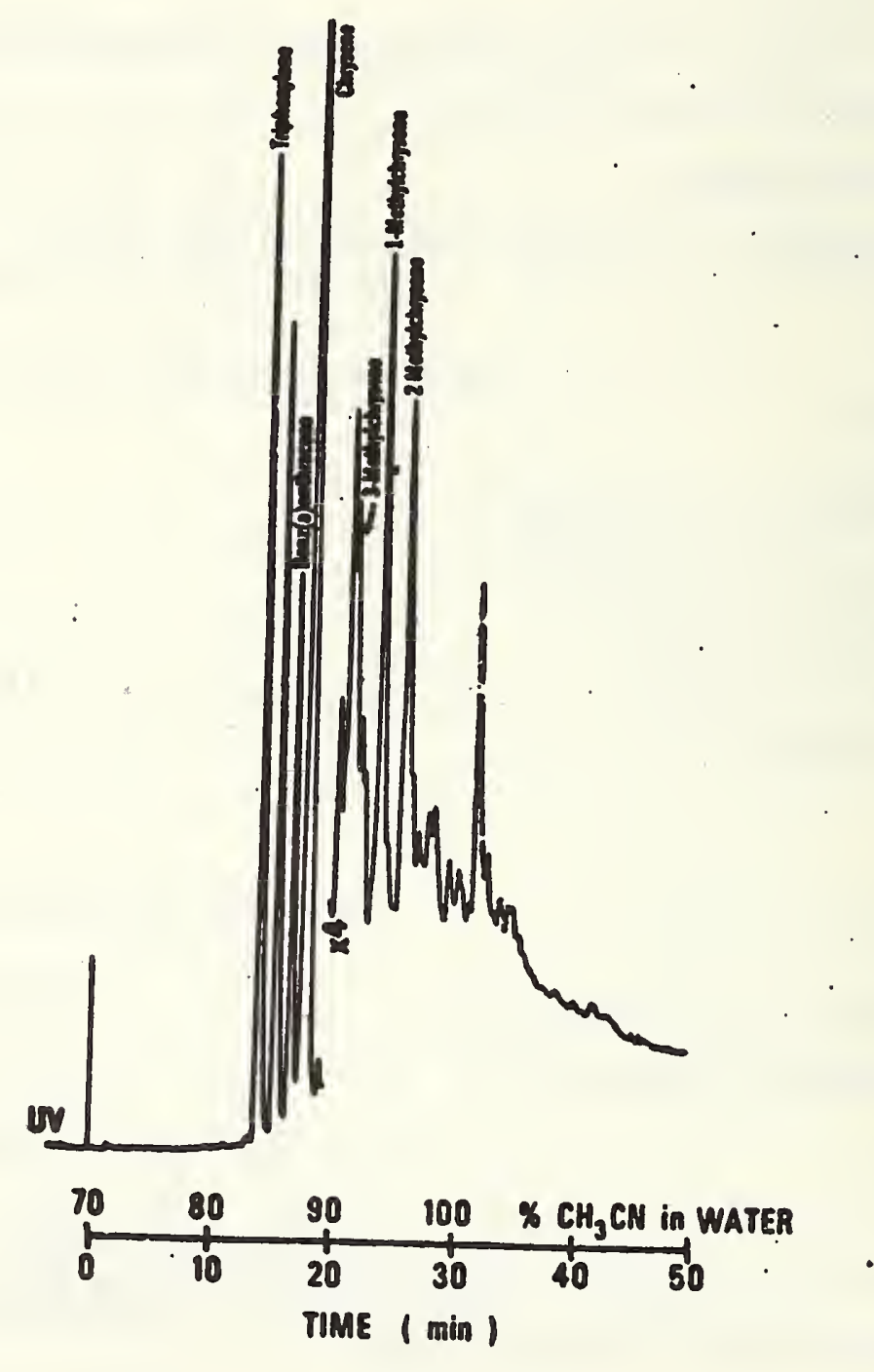

Figure 24. keverse phase 1iquid chromatographic analysis of fraction 4 from urban air particulates (see figure 8 ).

Column: Vydac 201TP reverse phase. Detection: UV absorbance at $254 \mathrm{~nm}$. Conditions: linear gradient from $70-100$ percent acetonitrile in water at $1 \% / \mathrm{min}$ at $1 \mathrm{~mL} / \mathrm{min}$. 
Participants: W. Zielinski, T. Flynn, J. Moody, R. Burke, D. Way

\section{Background:}

An Interagency project was developed in FY-1980 between NBS and the Department of the Treasury's Bureau of Engraving and Printing (BEP) at the request of BEP. This project, entitled "Provide Assistance to BEP in Assessing Potential Environmental Concerns and Prepare a Plan of Action Based on this Assessment", involves specific NBS competences in analytical chemistry and chemical engineering.

Activities planned under the established work plan for FY-1980 in analytical chemistry included: (a) the identification of BEP operational areas for chemical analysis; (b) chemical analysis of samples withdrawn from these areas for inorganics identified under the EPA hazardous wastes 1ist; (c) development of a survey report on BEP's chemical inventory log to assess any potential environmental concerns on chemicals used in BEP operations; and (d) provide technical assistance to BEP in its establishment of protocols for the management of specific wastes, as appropriate, to conform to EPA hazardous wastes guidelines.

Activities planned under the established work plan for FY-1980 in chemical engineering included: (a) the identification of BEP priority operational areas for chemical engineering evaluations related to potential environmental concerns, including the quantitative determination of operational capacities (present and potential) and the development of mass and energy balances for the priority areas; (b) carry out a detailed quantitative chemical engineering assessment the Dal-Mar press wash and effluent treatment system; (c) develop preliminary details for chemical engineering assessment of other areas identified by BEP; and (d) provide technical recommendations to BEP based on chemical engineering evaluations to enhance BEP's control and efficiency of operational systems.

NBS analytical chemistry activities related to environmental assessments for BEP are coordinated through the NBS Gaithersburg, Maryland facility in its NML Office of Environmental Measurements and Center for Analytical Chemistry (W. Zielinski, J. Moody, R. Burke), while NBS chemical engineering aitivities are carried out by chemical engineers at the NBS Boulder, Colorado facility (T. Flynn, D. Way). 
The following accomplishments were made in FY-1980 on this project:

O Finalization of FY-1980 work plan for chemical assessments and chemical engineering.

- Completion of survey of BEP chemical inventory log.

- Completion of preliminary chemical analyses on levels of the eight inorganics on the EPA hazardous wastes list $(\mathrm{Hg}, \mathrm{Cd}$, Se, As, $\mathrm{Cr}, \mathrm{Pb}, \mathrm{Ag}, \mathrm{Ba}$ ) on samples obtained from various areas at BEP.

- Completion of a detailed preliminary chemical engineering evaluation of BEP's Dal-Mar system.

- Establishment of preliminary data for chemical engineering evaluation of other BEP operational systems.

- Development of an approved work plan for FY-81, covering a continuing chemical analysis assessment and assistance towards making BEP self-sufficient in its analytical operations, chemical engineering assessment of additional areas identified by BEP, and including chemical engineering development for computer simulation of engineering processes at BEP to provide BEP with an internal control and flexibility for its processes. 
McClendon, Lottie T., "A Summary of $0 i 1$ Shale Activities at the National Bureau of Standards, 1975-1979," EPA 600/7-80-125, June 1980.

Kirchhoff, William H., "NBS/EPA Energy Environment Semi-Annual Report to EPA," July 1979.

May, Willie E., "Development of an Aqueous Trace Organic Standard Reference Material for Energy Related Applications," EPA 600/7-80-031, February 1980.

Kirchhoff, William H., "NBS/EPA Energy Environment Semi-Annual Report to EPA," May 1980.

Pella, Peter A., "The Development of Potention Thin Standards for Calibration of X-Ray Fluorescence Spectrometry," EPA 600/7-80-123, June 1980.

Kingston, H. M., "Quantitative Ultratrace Transition Metal Analysis of High Salinity Waters Utilizing Chelating Resin Separation," EPA 600/7-79-174, August 1979.

MacCrehan, W. A. and Durst, R. A., "Assessing the Environmental Impact of Fossi1-Fuel Based Energy Sources," EPA 600/8-79-211, November 1979.

Schwarz, Fred P., "Measurement of the Solubilities of Slightly Soluble Organic Liguids in Water by Elution Chromatography," Anal. Chem. 52, $10-15$ (1980).

Zielinski, Walter L., "Utility of High-Temperature Thermotropic Liquid Crystals as Stationary Phases for Novel Gas-Liquid Chromatographic Separations," J. Chromatogr. 186, 237-247 (1979).

Zielinski, Walter L., "Difficult Isomer Separations," Industrial Research/Development, 178-182, February 1980. 


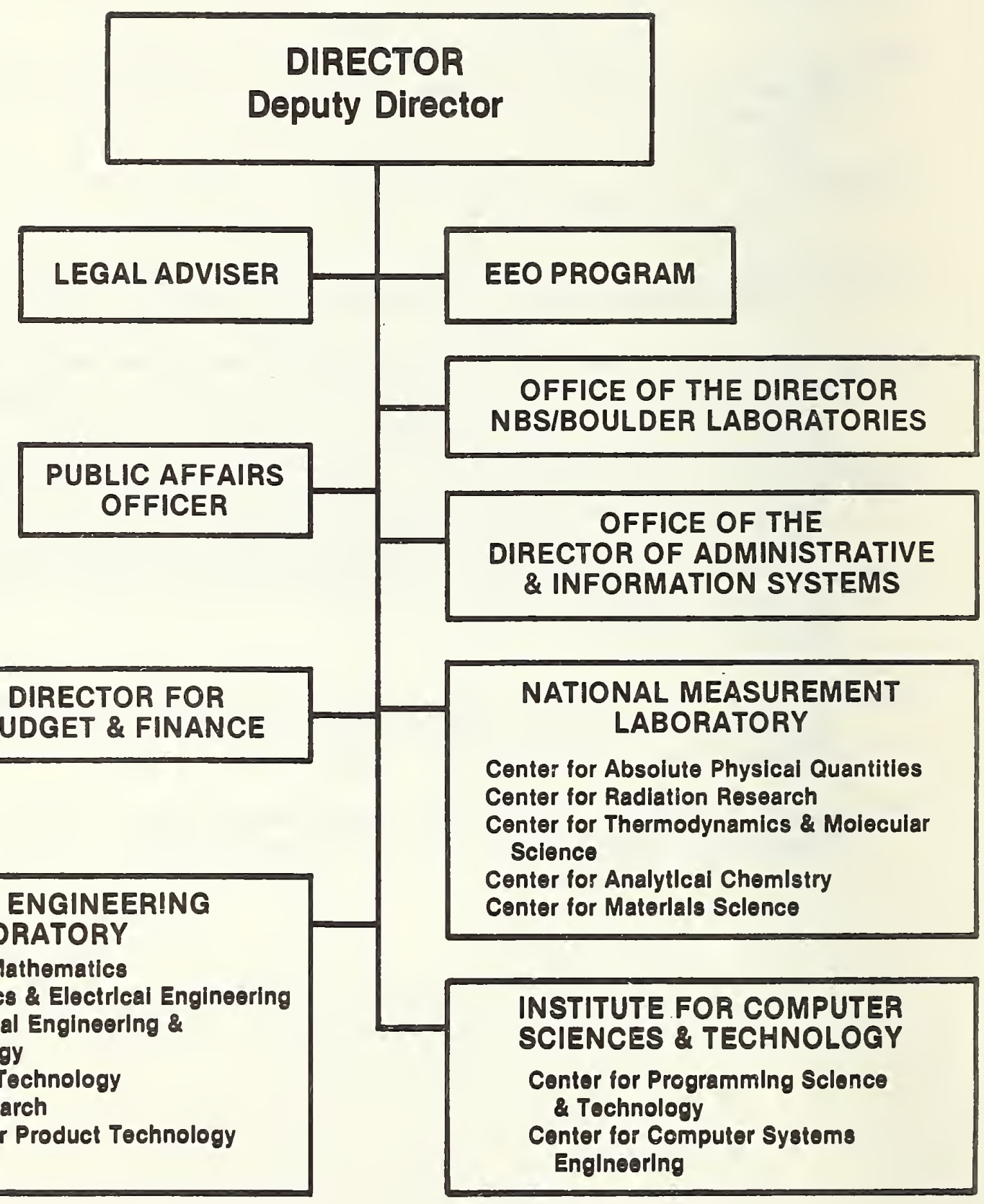




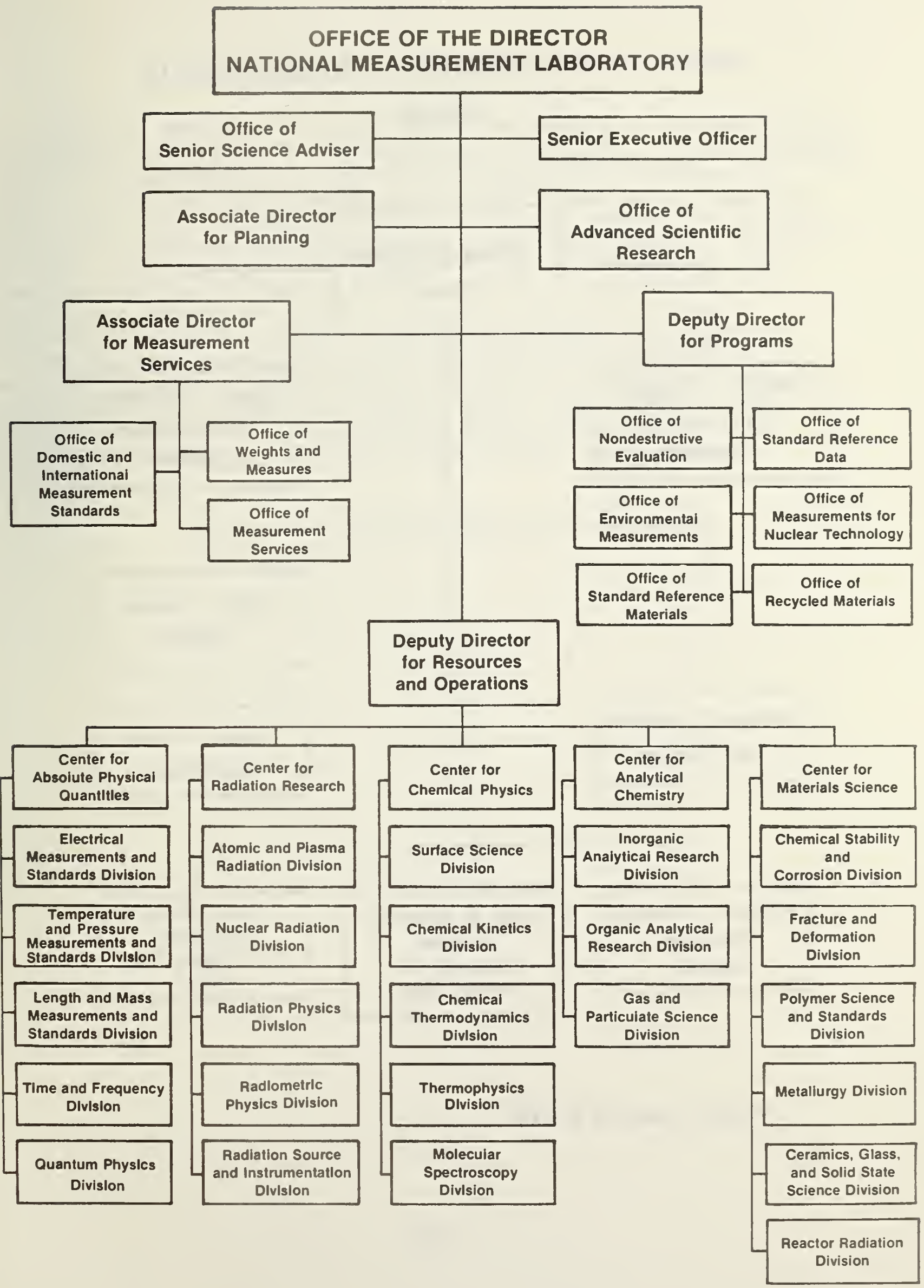


502.00

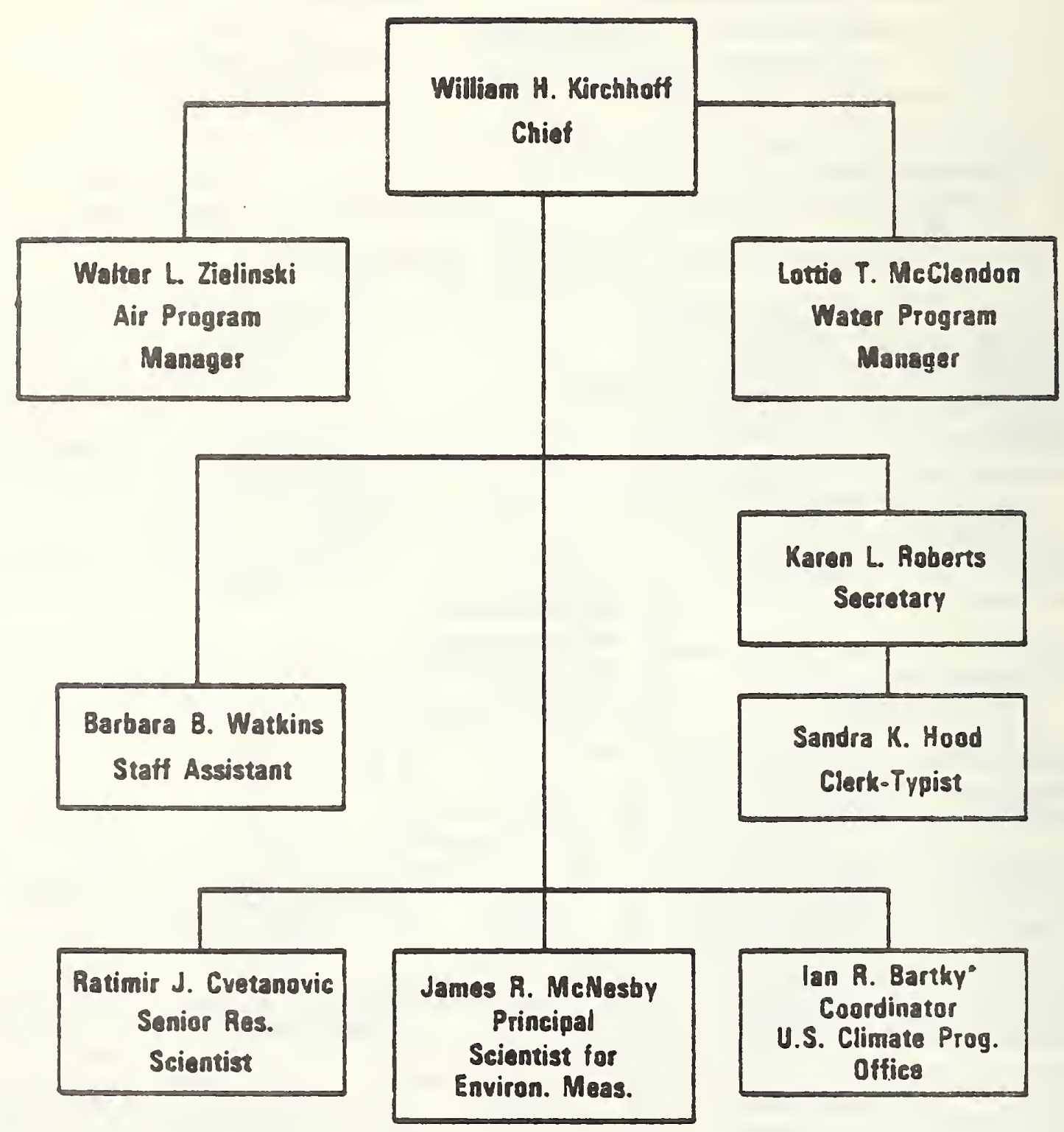

- Partially Supported by OEM. 
OFFICE OF ENVIRONMENTAL MEASUREMENTS: Responsibie for the development of standards and methods for the measurement of materials in the environment, such as the concentration of chemical pollutants in air and water, air "and water flow, and those chemical and physical properties of substances which affect their ultimate distribution in the environment; the provision of means for allowing measurement made by other Federal agencies, state, and local government agencies and industry to be consistent with these standards; and the provision of critically evaluated data on those physical and chemical properties of pollutants which affect their distribution in the environment. The NBS Environmental Measurements Program thus provides the accuracy and reliability basis for all air and water pollution, climate related, oceanographic, and toxic substances measurements made throughout the country by government (Federal, stata, and local) and industry. In addition, this office coordinates various international cooperative programs in environmental measurements and standards. 


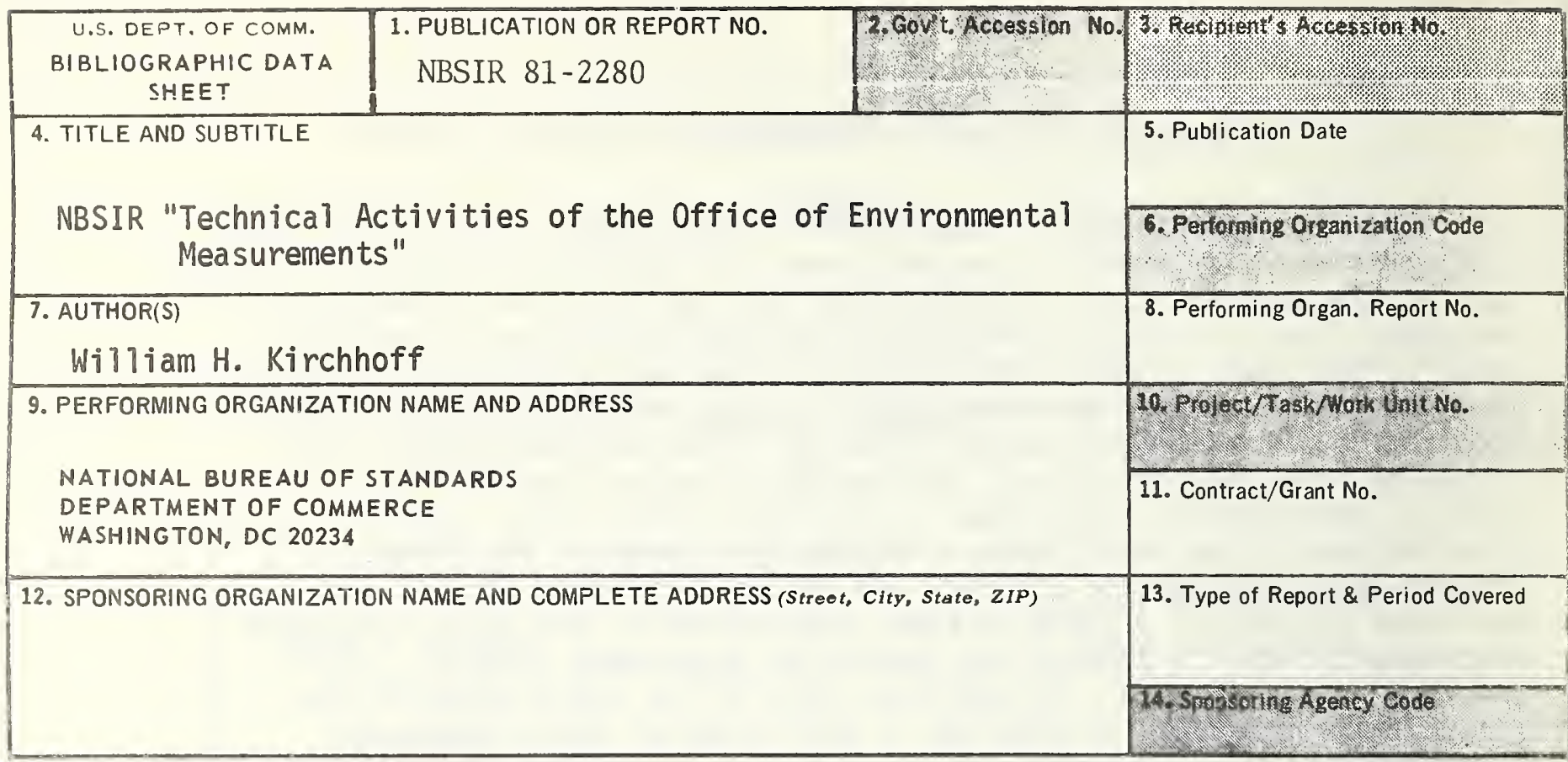

15. SUPPLEMENTARY NOTES

Document describes a computer program; SF-185, FIPS Software Summary, is attached.

16. ABSTRACT (A 200-word or less factual summary of most significant information. If documest includes a significant bibliography or literature survey, mention it here.)

This report summarizes the technical activities of the National Bureau of Standards' Environmental Measurements Program during Fiscal Year 1980. This activities fall into four program categories:

1) Water Measurements - Standards Development

2) Water Measurements - Methods Development

3) Air Measurements - Standards Development

4) Air Measurements - Methods Development

17. KEY WORDS (six to twelve entries; alphabetical order; capitalize only the first letter of the first key word unless a proper nams; separated by semicolons)

Air; water; environmental measurements; methods; quality assurance; standards

SECURITY CLASS (THIS REPORT)

20. SECURITY CLASS (THIS PAGE)

UNCLASSIFIED
UNCLASSIFIED

21. NO. OF PRINTED PAGES 192 VA. 22161 

